\title{
Well-defined Chiral Spiro Ir/Phosphine-oxazoline Cationic Complexes for Highly Enantioselective Hydrogenation of Imines at Ambient Pressure
}

\author{
Shou-Fei Zhu, Jian-Bo Xie, Yong-Zhen Zhang, Shen Li, Qi-Lin Zhou* \\ State Key Laboratory and Institute of Elemento-organic Chemistry, Nankai University \\ Tianjin 300071, China.
}

\section{CONTENTS:}

1. Analytical Data of Phosphine-oxazoline Ligands.

2. Analytical Data of Iridium Complexes.

3. X-Ray Analyses of Structures of Complexes $(S, S)-1$ a and $(S, R)-1 a$.

4. Preparation of Imine Substrates.

5. Asymmetric Hydrogenation of Imines and Analytical Data of Hydrogenation Products.

6. X-Ray Diffraction Analyses of Structure of 9e.

General. All reactions and manipulations were performed using standard Schlenk techniques. THF, diethyl ether, tert-butyl methyl ether (TBME), DME, toluene and hexane were distilled from sodium benzophenone ketyl. $\mathrm{CH}_{2} \mathrm{Cl}_{2}$ and DCE were washed with $98 \% \mathrm{H}_{2} \mathrm{SO}_{4}, \mathrm{H}_{2} \mathrm{O}$ and then distilled form $\mathrm{CaH}_{2}$ under an atmosphere of nitrogen. $\mathrm{Et}_{3} \mathrm{~N}$ were distilled from $\mathrm{CaH}_{2}$ under an atmosphere of nitrogen. DMSO was distilled over $\mathrm{CaH}_{2}$ under vacuum and stored under nitrogen. Methanol and ethanol were distilled over magnesium turnings under an atmosphere of nitrogen. $\mathrm{Pd}(\mathrm{OAc})_{2}$, 1,3-bis(diphenylphosphino)propane (dppp), $\mathrm{Pd}\left(\mathrm{PPh}_{3}\right)_{4}$ and $\mathrm{Zn}(\mathrm{CN})_{2}$ were purchased from Acros or Alderich Co. and used as received. $[\operatorname{Ir}(\mathrm{COD}) \mathrm{Cl}]_{2}$ was prepared from $\left(\mathrm{NH}_{4}\right)_{2} \mathrm{IrCl}_{6}$ or $\mathrm{IrCl}_{3} .3 \mathrm{H}_{2} \mathrm{O}$ according to the literatures. ${ }^{1} \mathrm{NaBARF} .3 \mathrm{H}_{2} \mathrm{O}$ 
was prepared according to the literatures. ${ }^{2}$ Melting points were measured on a RY-I apparatus and uncorrected. ${ }^{1} \mathrm{H},{ }^{13} \mathrm{C}$ and ${ }^{31} \mathrm{P}$ NMR spectra were recorded on Varian Mercury $400 \mathrm{MHz}$ or Bruker $300 \mathrm{MHz}$ spectrometers. Chemical shifts ( $\delta$ values) were reported in ppm down field from internal $\mathrm{Me}_{4} \mathrm{Si}\left({ }^{1} \mathrm{H}\right.$ and $\left.{ }^{13} \mathrm{C} \mathrm{NMR}\right)$ and external $85 \% \mathrm{H}_{3} \mathrm{PO}_{4}\left({ }^{31} \mathrm{P} \mathrm{NMR}\right)$ respectively. Optical rotations were determined using a Perkin Elmer 341 MC polarimeter. Elemental analyses were performed on Yanaca CDRDER MT-3 instrument. Mass spectra were recorded on a VG-7070E spectrometer. HPLC analyses were performed on a Hewlett Packard Model HP 1100 Series or a Waters 600E. X-Ray diffraction analysis was performed on a Bruker smart-1000 X-ray diffraction meter.

\section{Analytical Data of Phosphine-oxazoline Ligands}

(S)-7-Diphenylphosphino-7'-carbomethoxy-1,1'-spirobiindane ((S)-4a): Stiff oil and solidified by standing, 91\% yield. $[\alpha]^{20}{ }_{\mathrm{D}}-189.0$ (c 0.5, $\mathrm{CH}_{2} \mathrm{Cl}_{2}$ ); ${ }^{1} \mathrm{H} \mathrm{NMR}$ (300 MHz, $\mathrm{CDCl}_{3}$ ) $\delta 7.58$ (d, $J=7.8 \mathrm{~Hz}, 1 \mathrm{H}, \mathrm{Ar}-\mathrm{H}), 7.41$ (d, $J=8.4 \mathrm{~Hz}, 1 \mathrm{H}, \mathrm{Ar}-\mathrm{H}), 7.29-6.87$ (m, 14H, Ar-H), 3.12 (s, 3H, $\left.\mathrm{CH}_{3}\right), 3.10-2.91$ (m, 4H, $\left.\mathrm{CH}_{2}\right), 2.59-2.48\left(\mathrm{~m}, 1 \mathrm{H}, \mathrm{CH}_{2}\right), 2.34-2.20\left(\mathrm{~m}, 3 \mathrm{H}, \mathrm{CH}_{2}\right)$; ${ }^{31} \mathrm{P}$ NMR (121 MHz, $\left.\mathrm{CDCl}_{3}\right) \delta-20.8(\mathrm{~s}) ;{ }^{13} \mathrm{C} \mathrm{NMR}\left(75 \mathrm{MHz}, \mathrm{CDCl}_{3}\right) \delta 167.2,156.4,156.1$, 151.2, 145.9, 144.0, 143.9, 139.4, 139.2, 137.0, 136.8, 134.3, 134.0, 133.5, 133.3, 132.0, 131.6, 131.4, 131.2, 129.7, 128.8, 128.5, 128.3, 128.1, 127.7, 126.9, 126.7, 125.5, 64.0, 51.2, 41.0, 38.1, 31.2, 30.8. HRMS (FAB) calcd for $\mathrm{C}_{31} \mathrm{H}_{27} \mathrm{O}_{2} \mathrm{P}: 462.1743$, Found 462.1737.

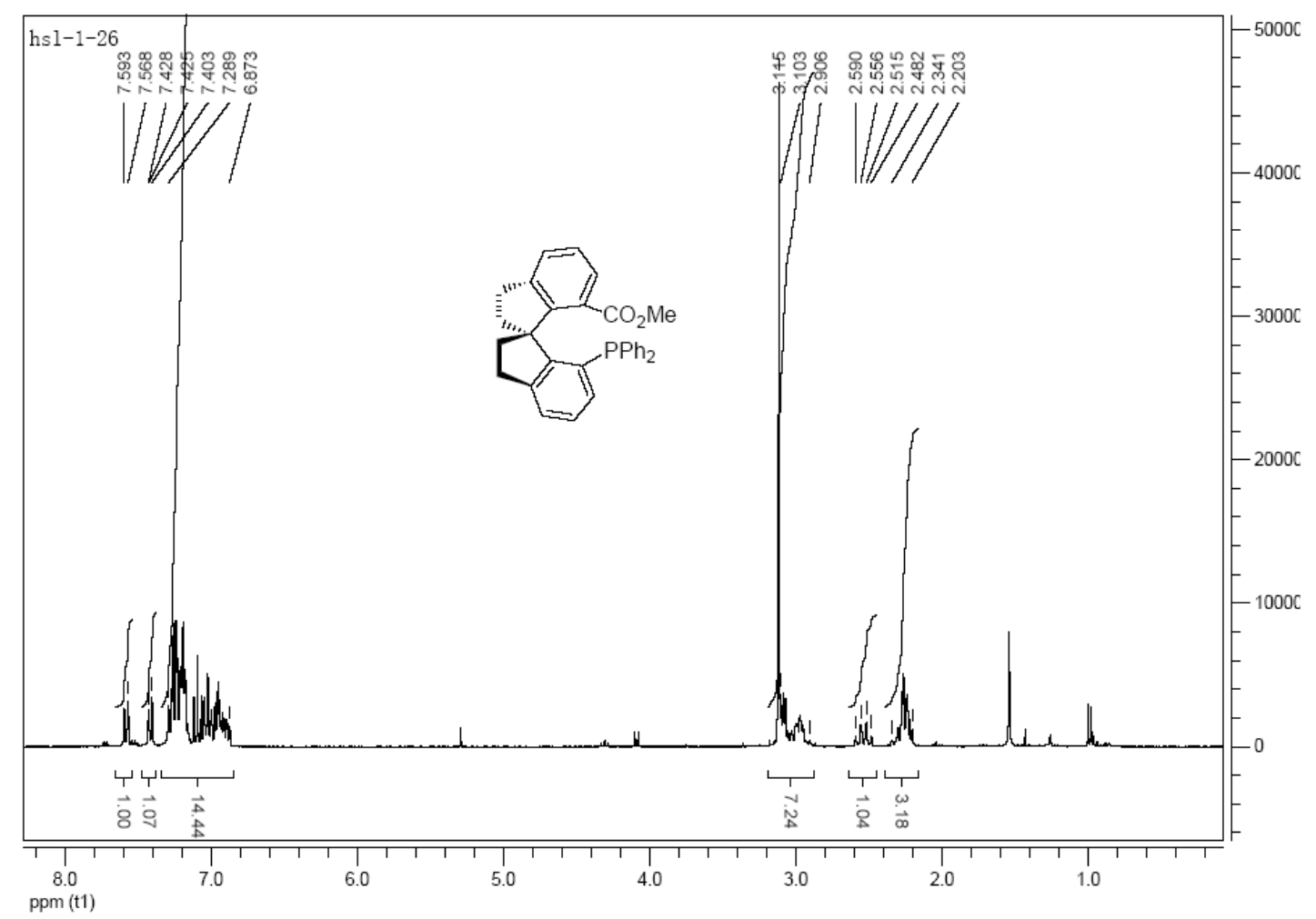




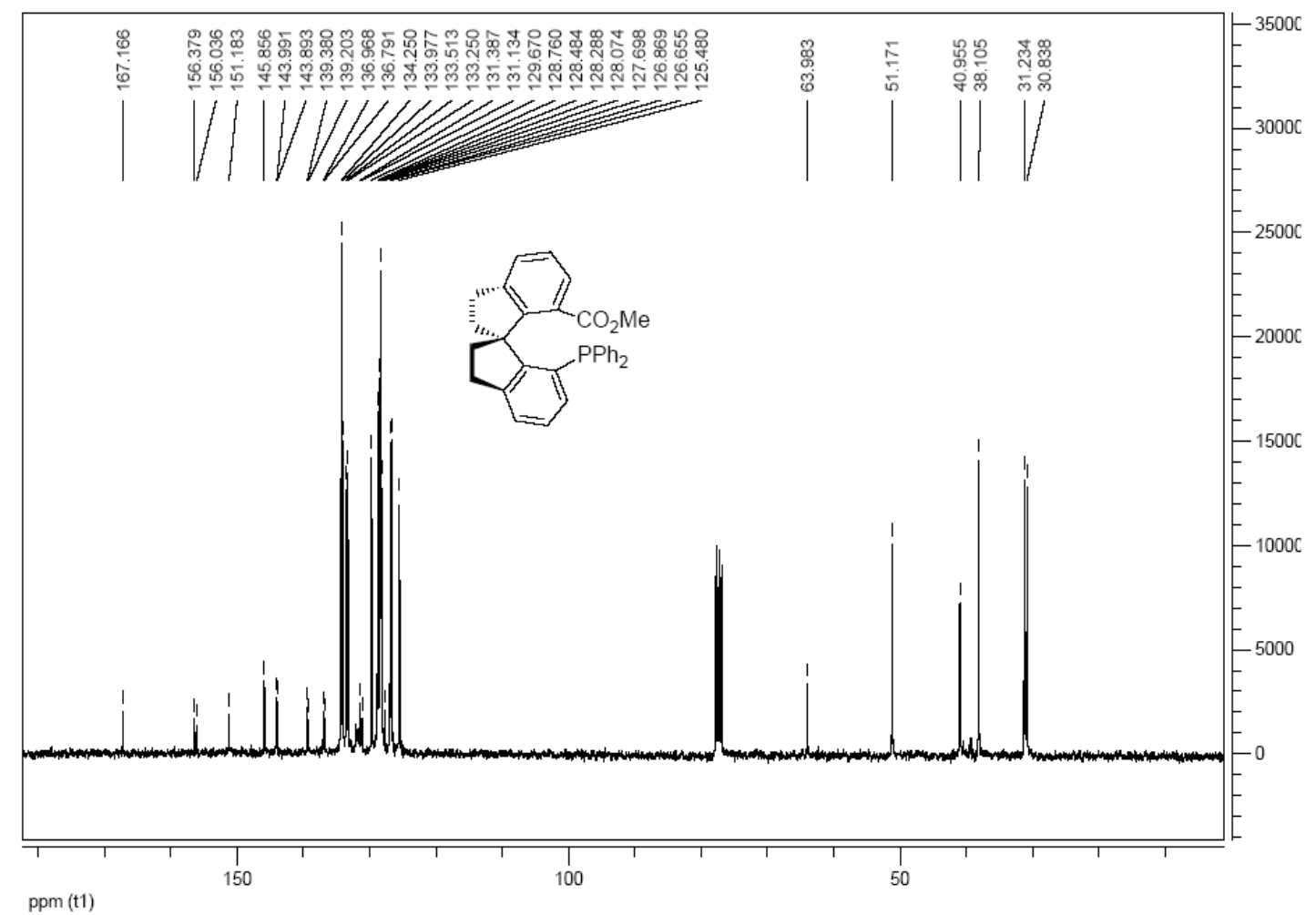

(S)-7-Di(4-methoxyphenyl)phosphino-7'-carbomethoxy-1,1'-spirobiindane

((S)-4b): White solid, 85\% yield. Mp 162-163 ${ }^{\circ} \mathrm{C}$; $[\alpha]^{20}{ }_{\mathrm{D}}-165.6$ (c 0.5, $\left.\mathrm{CH}_{2} \mathrm{Cl}_{2}\right) ;{ }^{1} \mathrm{H}$ NMR (300 MHz, $\left.\mathrm{CDCl}_{3}\right) \delta 7.61$ (d, $\left.J=7.5 \mathrm{~Hz}, 1 \mathrm{H}, \mathrm{Ar}-\mathrm{H}\right), 7.41$ (d, $\left.J=7.5 \mathrm{~Hz}, 1 \mathrm{H}, \mathrm{Ar}-\mathrm{H}\right), 7.27-7.22(\mathrm{~m}, 2 \mathrm{H}$, Ar-H), 7.08 (t, $J=7.5$ Hz, 1H, Ar-H), 6.97-6.78 (m, 5H, Ar-H), 6.75 (t, $J=9.0 \mathrm{~Hz}, 4 \mathrm{H}$ ), 3.78 (s, 3H, $\left.\mathrm{CH}_{3}\right), 3.74$ (s, 3H, $\left.\mathrm{CH}_{3}\right), 3.11$ (s, 3H, $\left.\mathrm{CH}_{3}\right), 3.08-3.06\left(\mathrm{~m}, 4 \mathrm{H}, \mathrm{CH}_{2}\right), 2.58-2.47$ (m, $1 \mathrm{H}, \mathrm{CH}_{2}$ ), 2.26-2.16 (m, 3H, $\left.\mathrm{CH}_{2}\right) ;{ }^{31} \mathrm{P}$ NMR (121 MHz, $\left.\mathrm{CDCl}_{3}\right) \delta-24.3(\mathrm{~s}) ;{ }^{13} \mathrm{C}$ NMR (75 $\left.\mathrm{MHz}, \mathrm{CDCl}_{3}\right) \delta 167.2,159.8,159.6,155.3,155.0,150.7,145.6,143.7,135.1,134.9,134.6$, 133.4, 132.5, 132.2, 130.0, 129.8, 129.2, 128.4, 128.1, 127.9, 126.6, 126.3, 125.0, 113.9, 113.8, 113.7, 113.6, 63.7, 55.2, 55.1, 50.9, 40.3, 38.0, 31.0, 30.6; MS (ESI) m/z 523 (M+1, 100); Anal. calcd for $\mathrm{C}_{33} \mathrm{H}_{31} \mathrm{O}_{4} \mathrm{P}$ : C 75.85, H 5.98; Found: C 76.04, H 5.93. 

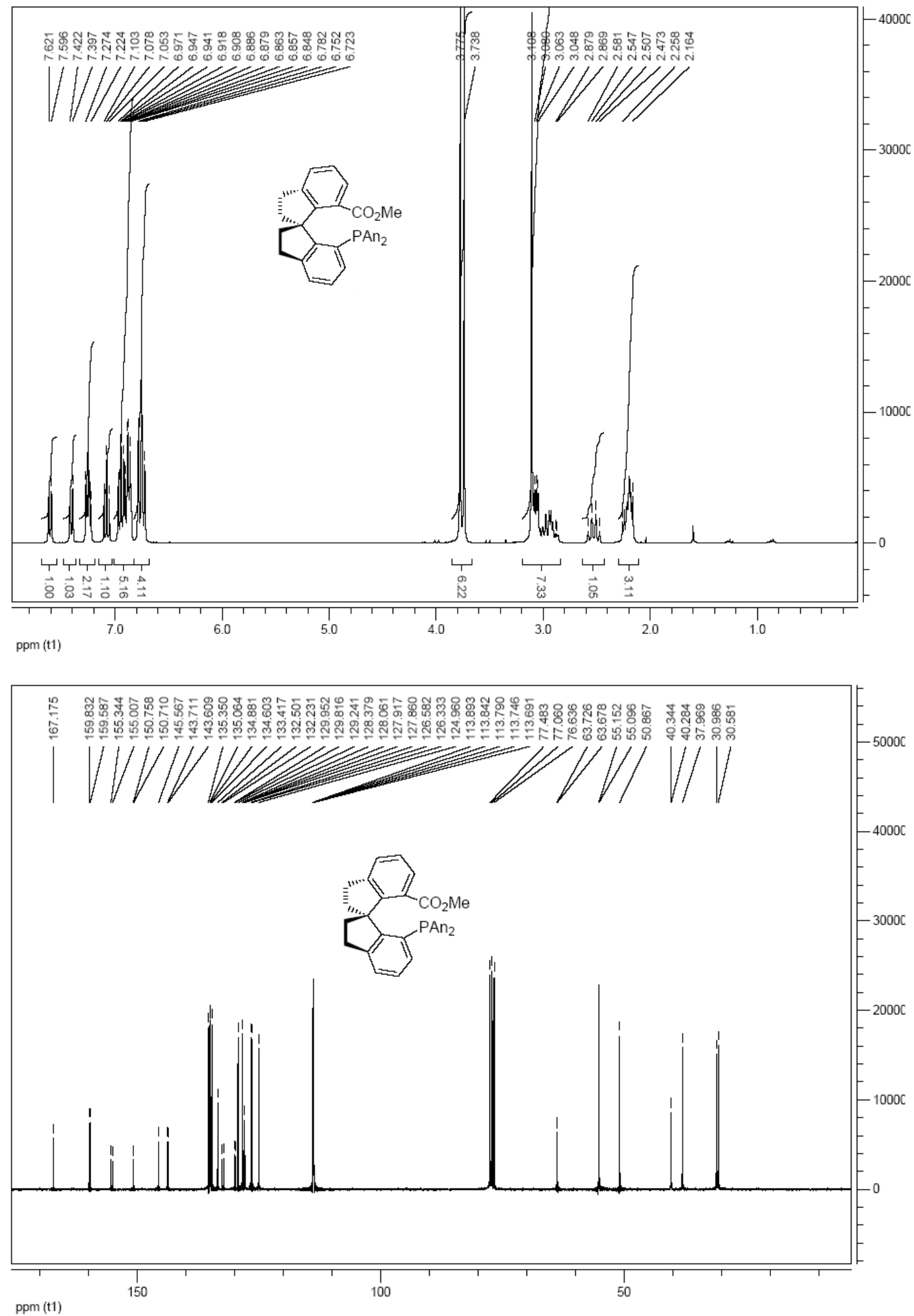

(S)-7-Di(3,5-dimethylphenyl)phosphino-7'-carbomethoxy-1,1'-spirobiindane $\quad$ ((S)-4c): White solid, 90\% yield. Mp 65-67 ${ }^{\circ} \mathrm{C}$; $[\alpha]^{20}{ }_{\mathrm{D}}-170.4$ (c 0.5, $\mathrm{CH}_{2} \mathrm{Cl}_{2}$ ); ${ }^{1} \mathrm{H}$ NMR $(300 \mathrm{MHz}$, $\left.\mathrm{CDCl}_{3}\right) \delta 7.56$ (d, $\left.J=7.8 \mathrm{~Hz}, 1 \mathrm{H}, \mathrm{Ar}-\mathrm{H}\right), 7.42$ (dd, $J=7.2$ and $0.9 \mathrm{~Hz}, 1 \mathrm{H}, \mathrm{Ar}-\mathrm{H}$ ), 7.27-7.12 (m, 2H, Ar-H), 7.09 (t, $J=7.5$ Hz, 1H, Ar-H), 6.97-6.93 (m, 1H, Ar-H), 6.88 (s, 1H, Ar-H), 6.82 (s, 1H, Ar-H), 6.58 (dd, $J=9.9$ and $9.0 \mathrm{~Hz}, 4 \mathrm{H}, \mathrm{Ar}-\mathrm{H}), 3.09$ (s, 3H, $\mathrm{CH}_{3}$ ), 3.06-2.99 (m, $\left.4 \mathrm{H}, \mathrm{CH}_{2}\right), 2.52-2.40\left(\mathrm{~m}, 2 \mathrm{H}, \mathrm{CH}_{2}\right), 2.37-2.29\left(\mathrm{~m}, 2 \mathrm{H}, \mathrm{CH}_{2}\right), 2.21\left(\mathrm{~s}, 6 \mathrm{H}, \mathrm{CH}_{3}\right), 2.16(\mathrm{~s}, 6 \mathrm{H}$, $\left.\mathrm{CH}_{3}\right) ;{ }^{31} \mathrm{P}$ NMR $\left(121 \mathrm{MHz}, \mathrm{CDCl}_{3}\right) \delta-20.8(\mathrm{~s}) ;{ }^{13} \mathrm{C} \mathrm{NMR}\left(75 \mathrm{MHz}, \mathrm{CDCl}_{3}\right) \delta 166.6,156.3$, 
156.0, 151.2, 151.1, 145.8, 143.3, 143.2, 138.9, 138.8, 137.2, 137.1, 137.0, 136.0, 134.0, 132.0, 131.8, 131.7, 131.6, 131.0, 130.9, 130.6, 130.1, 130.0, 129.5, 129.4, 128.5, 127.4, 126.3, 126.2, 124.9, 63.9, 50.7, 40.7, 38.3, 31.0, 30.7, 21.4, 21.3; MS (ESI) m/z 519 (M+1, 100); Anal. calcd for $\mathrm{C}_{35} \mathrm{H}_{35} \mathrm{O}_{2} \mathrm{P}$ : C 81.06, H 6.80; Found: C 80.95, H 6.94.

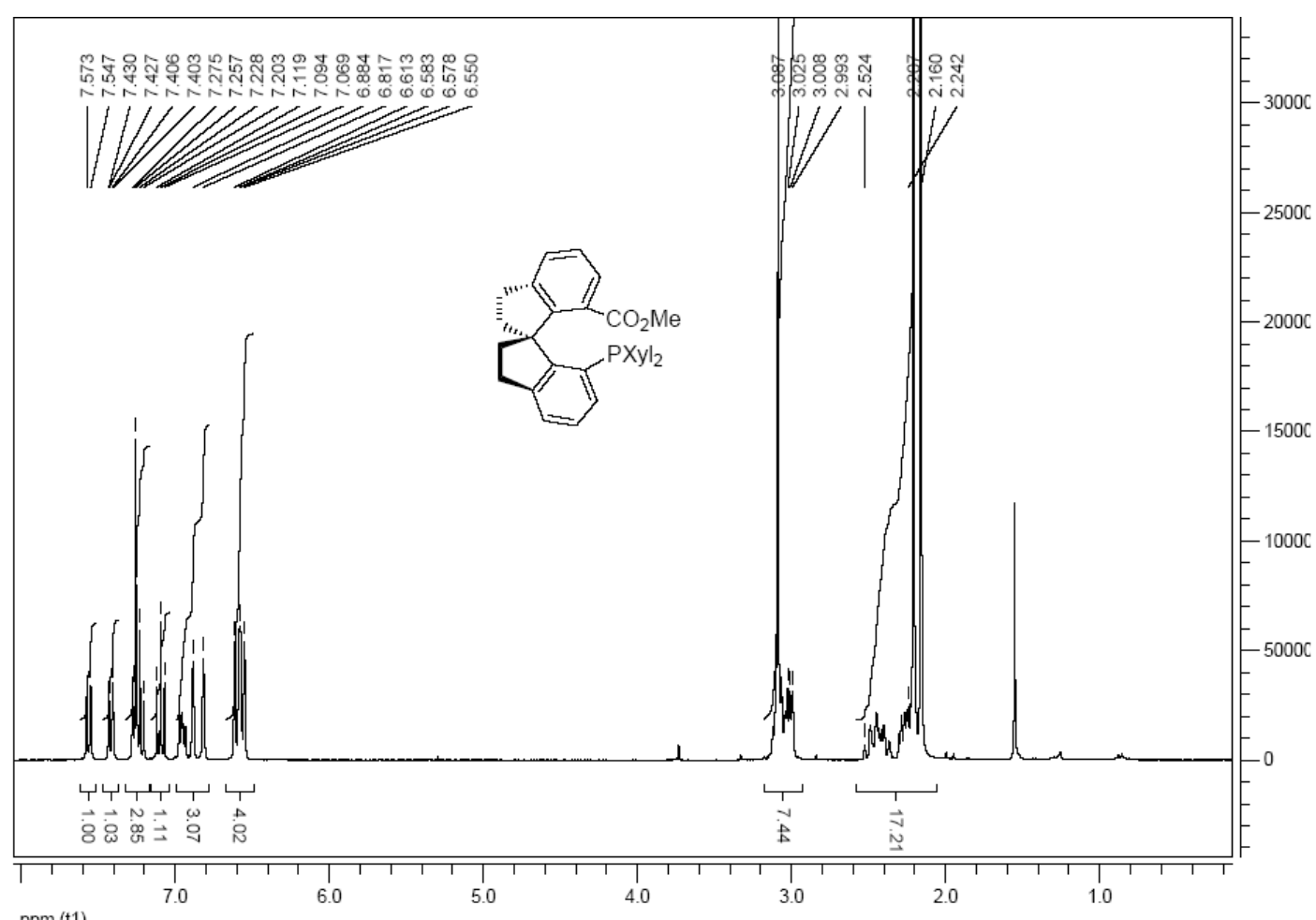

$\operatorname{ppm}(\mathrm{t} 1)$

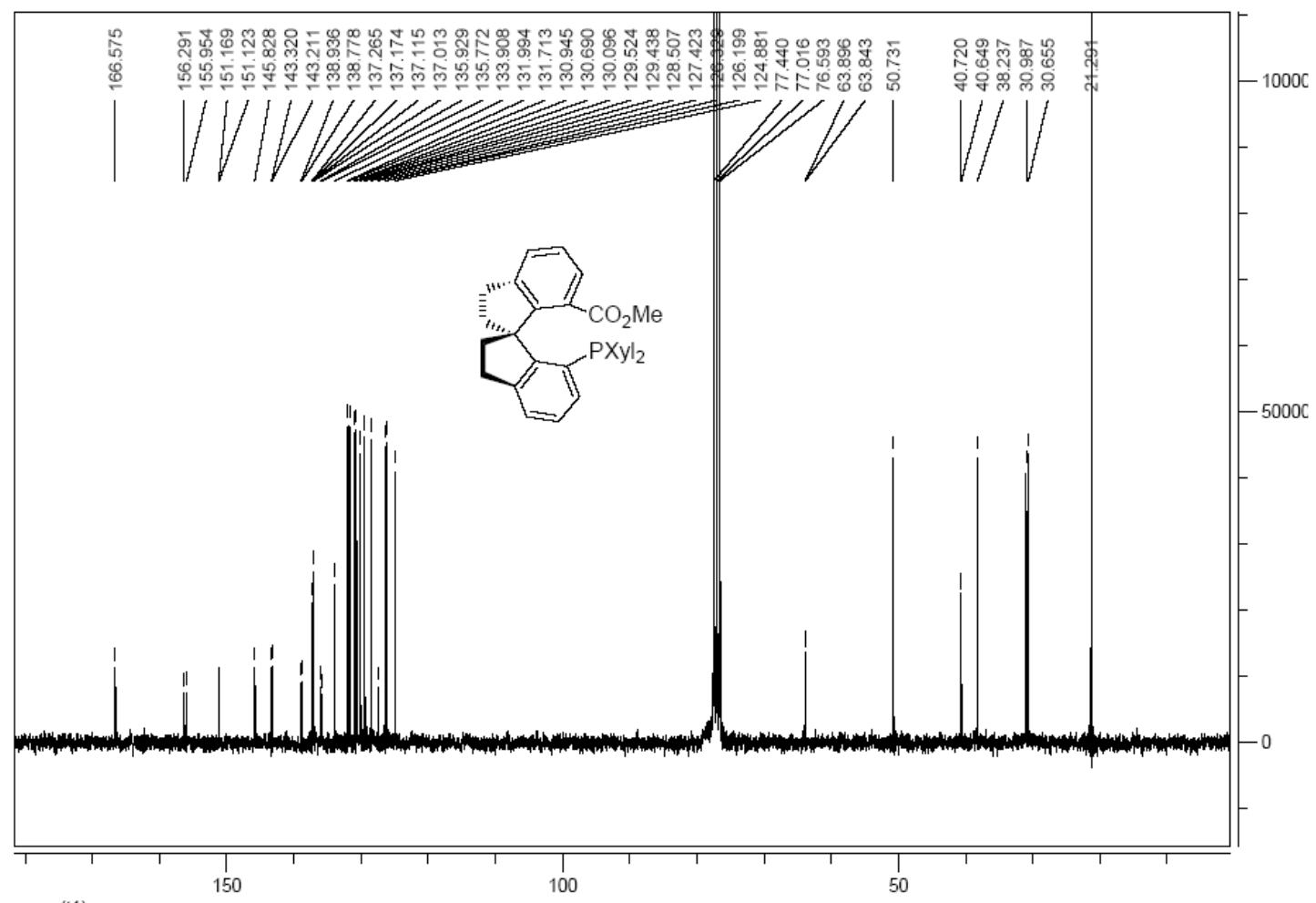

ppm (t1) 
(S)-7-Diphenylphosphino-7'-carboxy-1,1'-spirobiindane ((S)-5a): White solid, 99\% yield. Mp 202-203 ${ }^{\circ} \mathrm{C} ;[\alpha]^{20}{ }_{\mathrm{D}}-153.4\left(c\right.$ 0.5, $\left.\mathrm{CH}_{2} \mathrm{Cl}_{2}\right) ;{ }^{1} \mathrm{H}$ NMR (300 MHz, $\left.\mathrm{CDCl}_{3}\right) \delta$ 7.54-6.82 (m, 16H, Ar-H), 3.13-3.02 (m, 4H, CH $)$, 2.55-2.19 (m, 4H, $\left.\mathrm{CH}_{2}\right) ;{ }^{31} \mathrm{P}$ NMR (121 MHz, $\mathrm{CDCl}_{3}$ ) $\delta-20.8(\mathrm{~s}) ;{ }^{13} \mathrm{C}$ NMR $\left(75 \mathrm{MHz}, \mathrm{CDCl}_{3}\right) \delta 170.7,156.8,156.4,152.7,146.2,144.2,144.4$, 139.7, 139.5, 136.3, 134.2, 134.0, 133.7, 133.4, 133.1, 130.7, 130.3, 129.6, 128.5, 128.4, 128.2, 127.9, 126.8, 126.6, 126.0, 125.3, 64.0, 41.5, 37.7, 31.4, 30.9; HRMS (FAB) calcd for $\mathrm{C}_{30} \mathrm{H}_{25} \mathrm{O}_{2} \mathrm{P}$ : 448.1592; Found 448.1587.
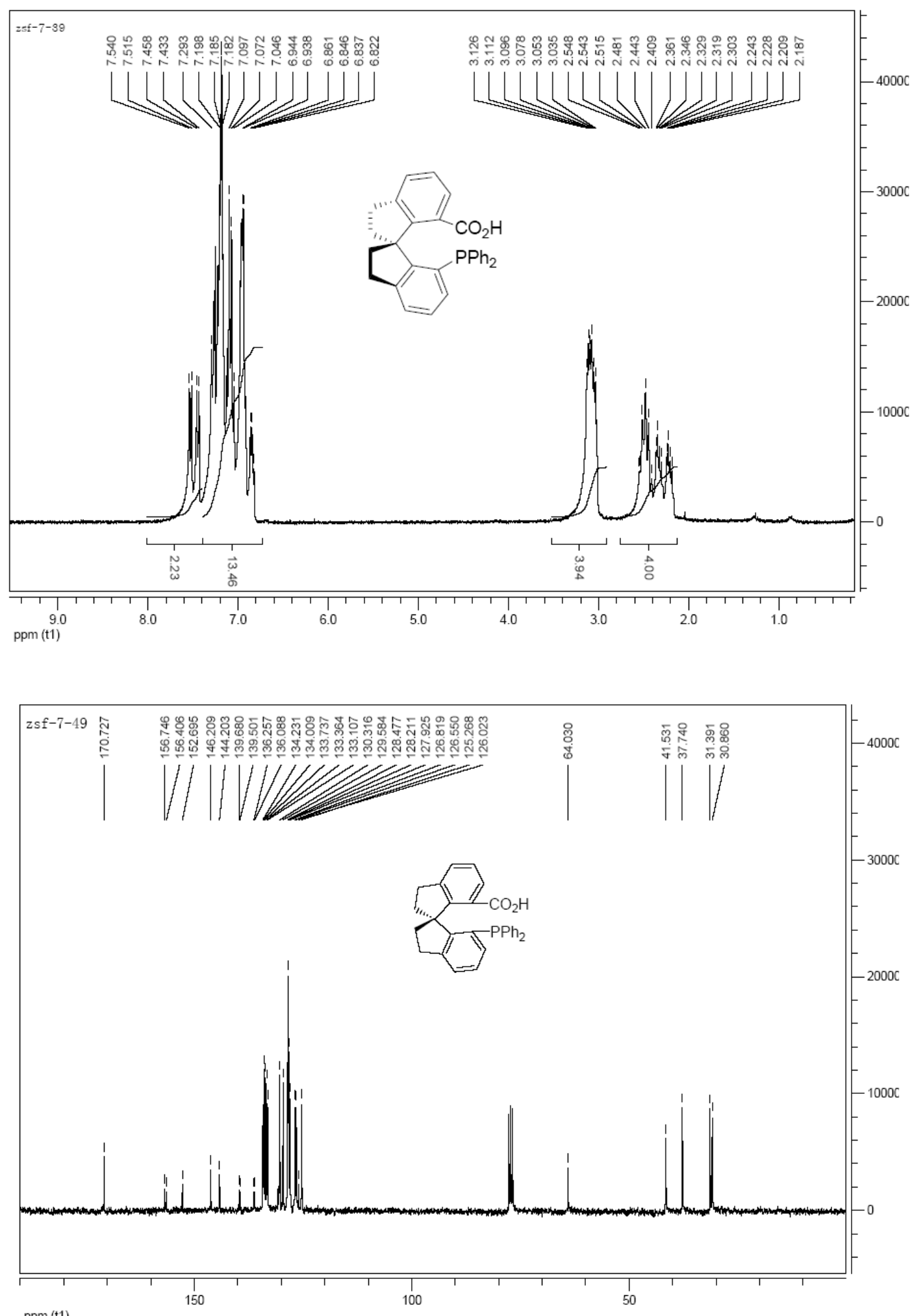
(S)-7-Di(4-methoxyphenyl)phosphino-7'-carboxy-1,1'-spirobiindane ((S)-5b): White solid, 67\% yield. Mp 183-184 ${ }^{\circ} \mathrm{C}$; $[\alpha]^{20}{ }_{\mathrm{D}}-154.8$ (c 0.5, $\left.\mathrm{CH}_{2} \mathrm{Cl}_{2}\right) ;{ }^{1} \mathrm{H}$ NMR (300 $\left.\mathrm{MHz}, \mathrm{CDCl}_{3}\right) \delta$ 7.58 (d, $J=6.9 \mathrm{~Hz}, 1 \mathrm{H}$, Ar-H); 7.44 (d, $J=7.2 \mathrm{~Hz}, 1 \mathrm{H}, \mathrm{Ar}-\mathrm{H}$ ), 7.25-7.20 (m, 2H, Ar-H), 7.03 (t, $J=7.5 \mathrm{~Hz}, 1 \mathrm{H}, \mathrm{Ar}-\mathrm{H}), 6.89-6.66$ (m, 9H, Ar-H), 3.75 (d, $J=7.5 \mathrm{~Hz}, 6 \mathrm{H}, \mathrm{CH}_{3}$ ), 3.08-2.98 (m, 4H, CH $\mathrm{CH}_{2}$, 2.49-2.15 (m, 4H, $\mathrm{CH}_{2}$ ); ${ }^{31} \mathrm{P}$ NMR (121 MHz, $\mathrm{CDCl}_{3}$ ) $\delta-24.1$ (s); ${ }^{13} \mathrm{C} \mathrm{NMR}$ $\left(100 \mathrm{MHz}, \mathrm{CDCl}_{3}\right) \delta 170.5,160.1,159.7,152.4,146.1,143.9,135.4,135.2,134.9,134.7$, 133.6, 132.0, 130.4, 130.1, 129.4, 127.5, 126.8, 125.4, 114.1, 113.9, 64.0, 55.3, 41.0, 37.7, 31.3, 30.8; MS (ESI) m/z 507 (M-1, 100); Anal. calcd for $\mathrm{C}_{32} \mathrm{H}_{29} \mathrm{O}_{4} \mathrm{P}: \mathrm{C}$ 75.58, H 5.75; Found: C 75.00, H 5.95.

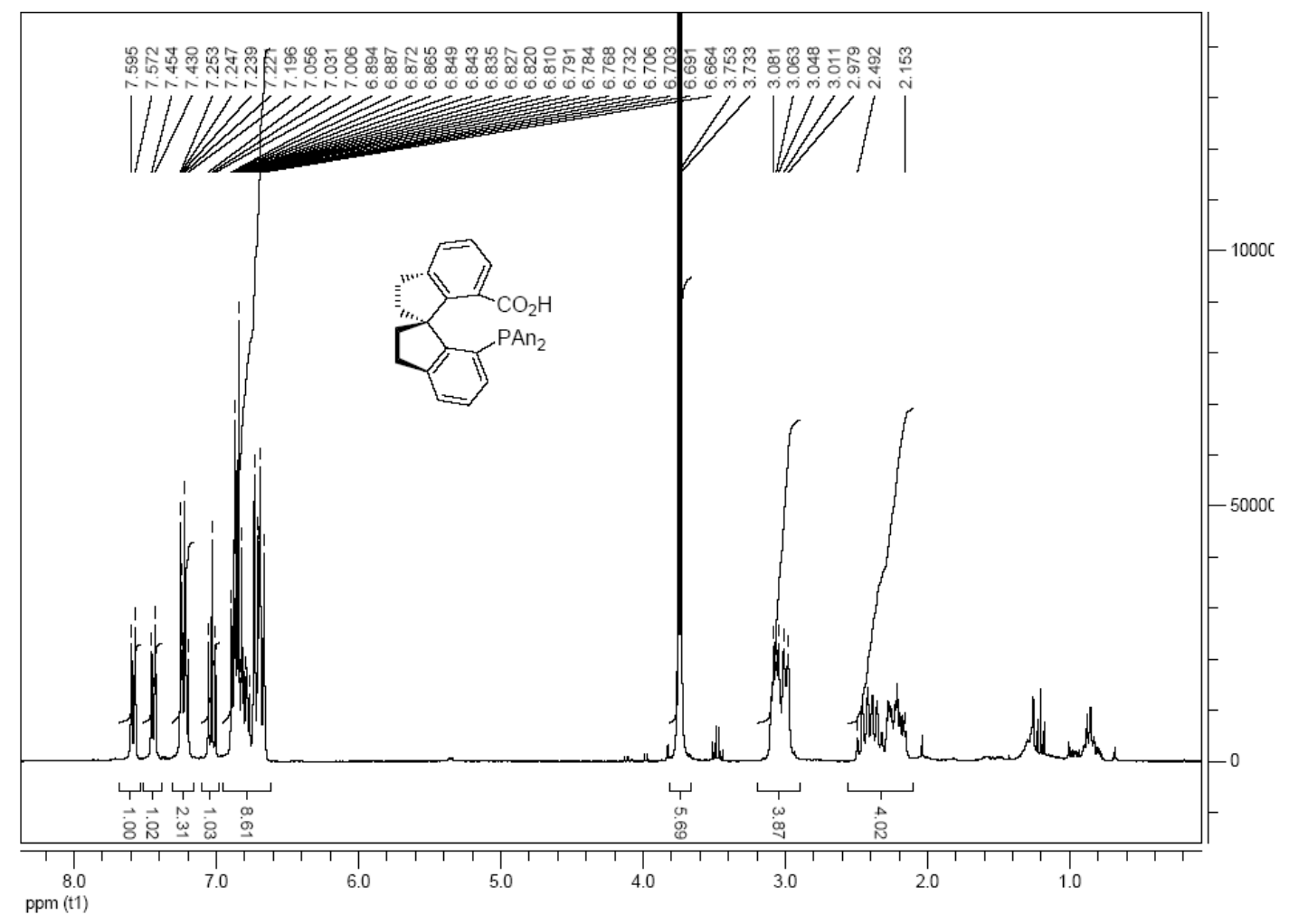




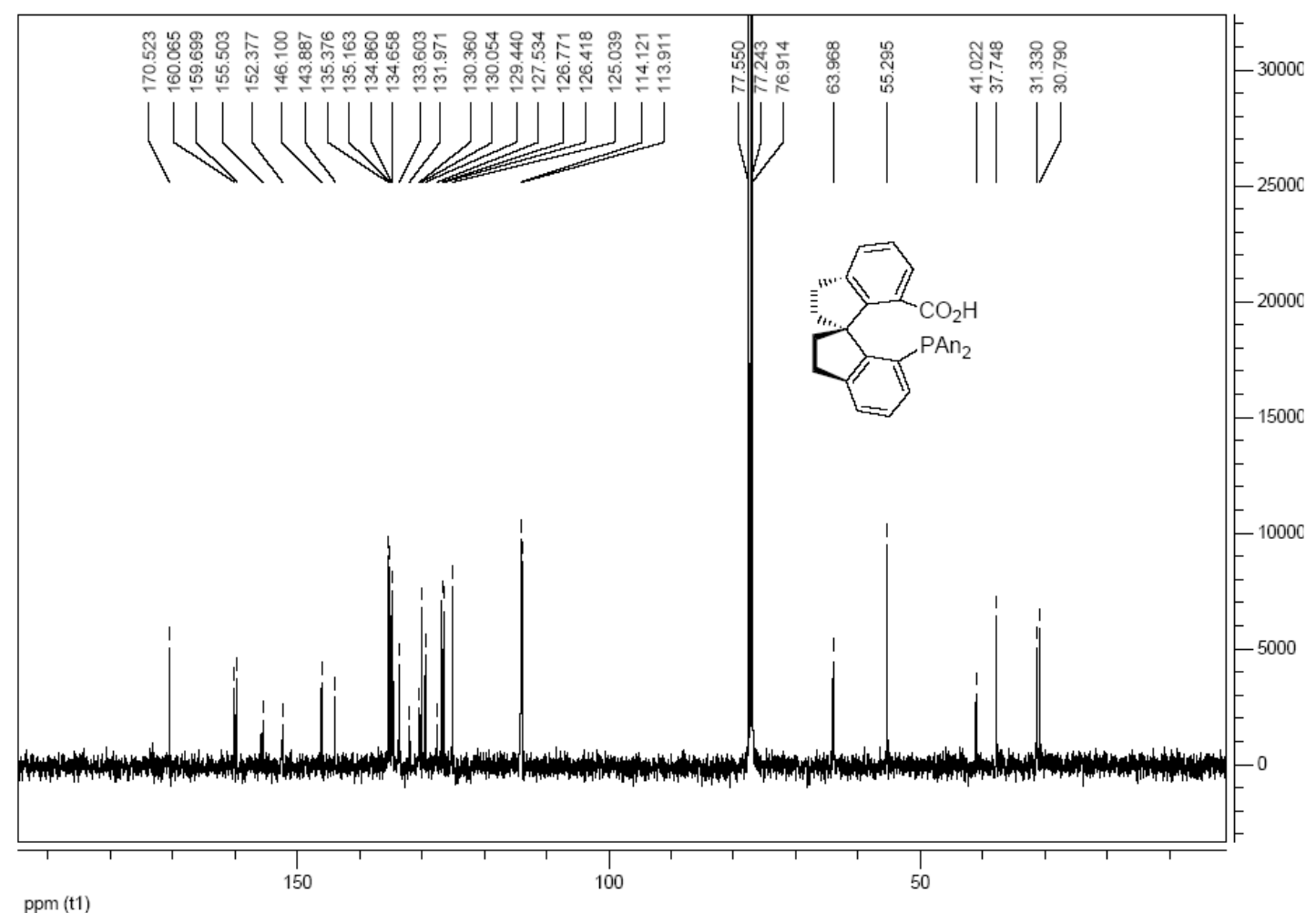

(S)-7-Di(3,5-dimethylphenyl)phosphino-7'-carboxy-1,1'-spirobiindane ((S)-5c): White solid, 90\% yield. Mp 110-112 ${ }^{\circ} \mathrm{C}$; $[\alpha]^{20}{ }_{\mathrm{D}}-143.2\left(\mathrm{c} 0.5, \mathrm{CH}_{2} \mathrm{Cl}_{2}\right) ;{ }^{1} \mathrm{H}$ NMR (300 MHz, $\left.\mathrm{CDCl}_{3}\right)$ $\delta 7.49$ (d, $J=7.8 \mathrm{~Hz}, 1 \mathrm{H}, \mathrm{Ar}-\mathrm{H}$ ), 7.42 (d, $J=7.2 \mathrm{~Hz}, 1 \mathrm{H}, \mathrm{Ar}-\mathrm{H}), 7.26-7.16$ (m, 2H, Ar-H); 7.04 (t, $J=7.5 \mathrm{~Hz}, 1 \mathrm{H}, \mathrm{Ar}-\mathrm{H}), 6.86-6.80$ (m, 3H, Ar-H), 6.54 (dd, $J=15$ and $7.5 \mathrm{~Hz}, 4 \mathrm{H}$, Ar-H), 3.12-3.01 (m, 4H, CH $), 2.56-2.19$ (m, 4H, $\left.\mathrm{CH}_{2}\right), 2.15$ (s, 6H, $\left.\mathrm{CH}_{3}\right), 2.10$ (s, 6H, $\left.\mathrm{CH}_{3}\right) ;{ }^{31} \mathrm{P}$ NMR (121 MHz, $\left.\mathrm{CDCl}_{3}\right) \delta-20.6(\mathrm{~s}) ;{ }^{13} \mathrm{C} \mathrm{NMR}\left(100 \mathrm{MHz}, \mathrm{CDCl}_{3}\right) \delta 170.3,156.1$, 155.7, 152.4, 146.1, 146.0, 143.7, 143.6, 139.0, 138.8, 137.2, 137.1, 135.7, 135.6, 133.8, 131.7, 131.5, 130.8, 130.0, 129.9, 129.5, 129.1, 126.3, 124.7, 63.8, 41.3, 41.2, 37.9, 31.2, 30.7, 21.3, 21.2; HRMS (EI) calcd for $\mathrm{C}_{34} \mathrm{H}_{33} \mathrm{O}_{2} \mathrm{P}$ : 504.2218; Found 504.2223. 

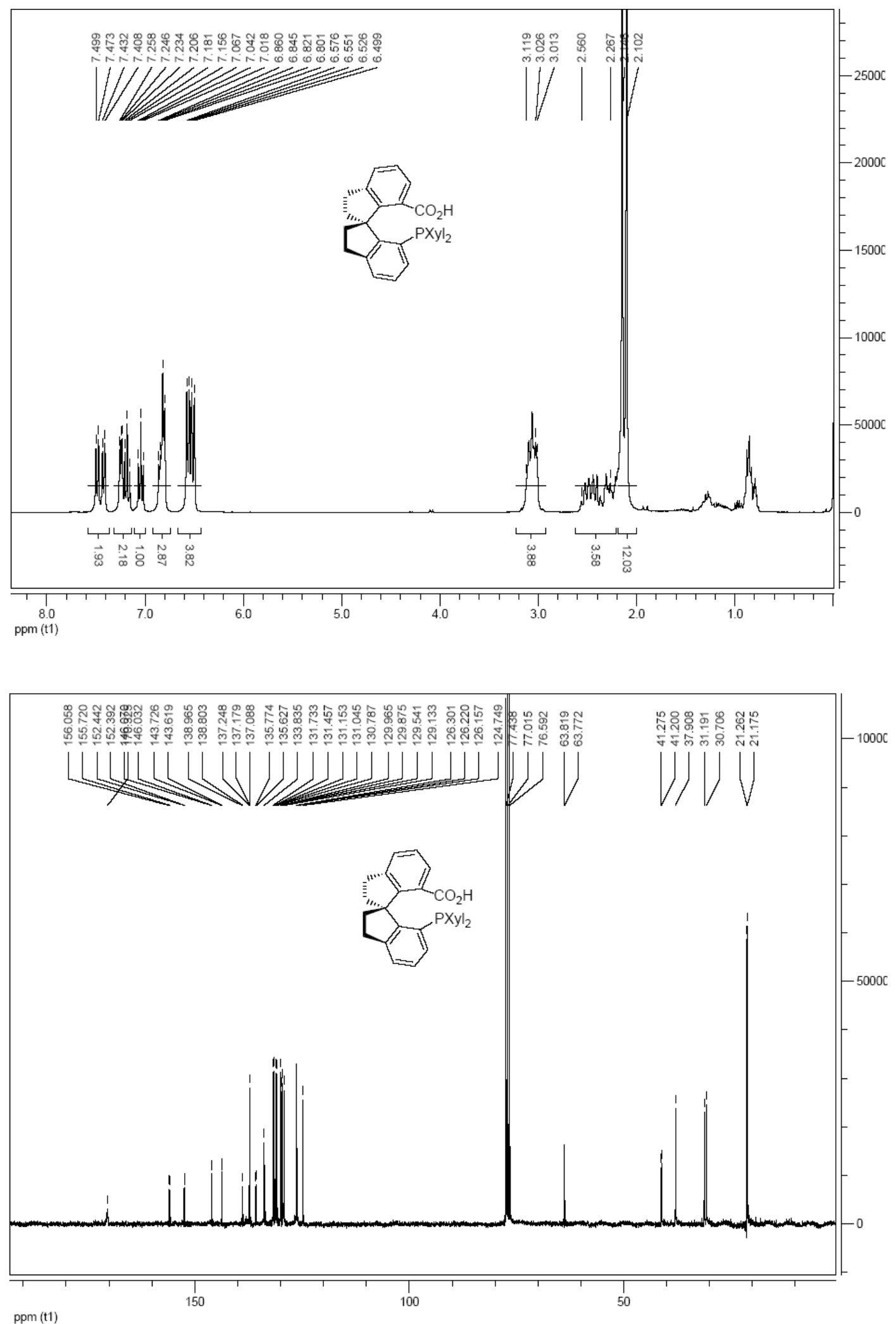

(S,S)-N-(1-Hydroxymethyl-2-methylpropyl)-7'-diphenylphosphino-1,1'-spirobiindane-7carboxylamide $((\boldsymbol{S}, \boldsymbol{S})-\mathbf{6 a})$ : Stiff oil and solidified with standing, $100 \%$ yield. $[\alpha]^{20}{ }_{\mathrm{D}}-193.6$ (c 0.5, $\mathrm{CH}_{2} \mathrm{Cl}_{2}$ ); ${ }^{1} \mathrm{H}$ NMR (400 MHz, $\mathrm{CDCl}_{3}$ ) $\delta$ 7.37-6.93 (m, $\left.16 \mathrm{H}, \mathrm{Ar}-\mathrm{H}\right), 4.80$ (d, $J=6.8 \mathrm{~Hz}$, $1 \mathrm{H}, \mathrm{NH}), 3.67$ (s, 1H, OH), 3.35-3.32 (m, 1H, CH), 3.21-2.88 (m, 6H, $\left.\mathrm{CH}_{2}\right), 2.88-2.70(\mathrm{~m}$, $1 \mathrm{H}, \mathrm{CH}_{2}$ ), 2.63-2.27 (m, 3H, $\left.\mathrm{CH}_{2}\right), 1.60-1.51(\mathrm{~m}, 1 \mathrm{H}, \mathrm{CH}), 0.67$ (d, $J=6.8 \mathrm{~Hz}, 3 \mathrm{H}, \mathrm{CH}_{3}$ ), 0.59 (d, $\left.J=6.4 \mathrm{~Hz}, 3 \mathrm{H}, \mathrm{CH}_{3}\right) ;{ }^{31} \mathrm{P}$ NMR $\left(161 \mathrm{MHz}, \mathrm{CDCl}_{3}\right) \delta-20.0(\mathrm{~s}) ;{ }^{13} \mathrm{C}$ NMR $(100 \mathrm{MHz}$, 
$\left.\mathrm{CDCl}_{3}\right) \delta 170.8,156.0,155.7,149.4,146.4,145.4,140.2,137.5,137.2,135.5,134.2,133.7$, 133.0, 132.7, 132.5, 129.5, 128.6, 128.3, 127.1, 126.6, 126.0, 63.2, 60.8, 42.9, 40.5, 31.2, 28.2, 20.2, 19.2; MS (ESI) m/z 532 (M-1, 44); Anal. calcd for $\mathrm{C}_{35} \mathrm{H}_{36} \mathrm{NO}_{2} \mathrm{P}: \mathrm{C}$ 78.77, $\mathrm{H}$ 6.80, N 2.62; Found: C 78.67, H 6.69, N 2.89.
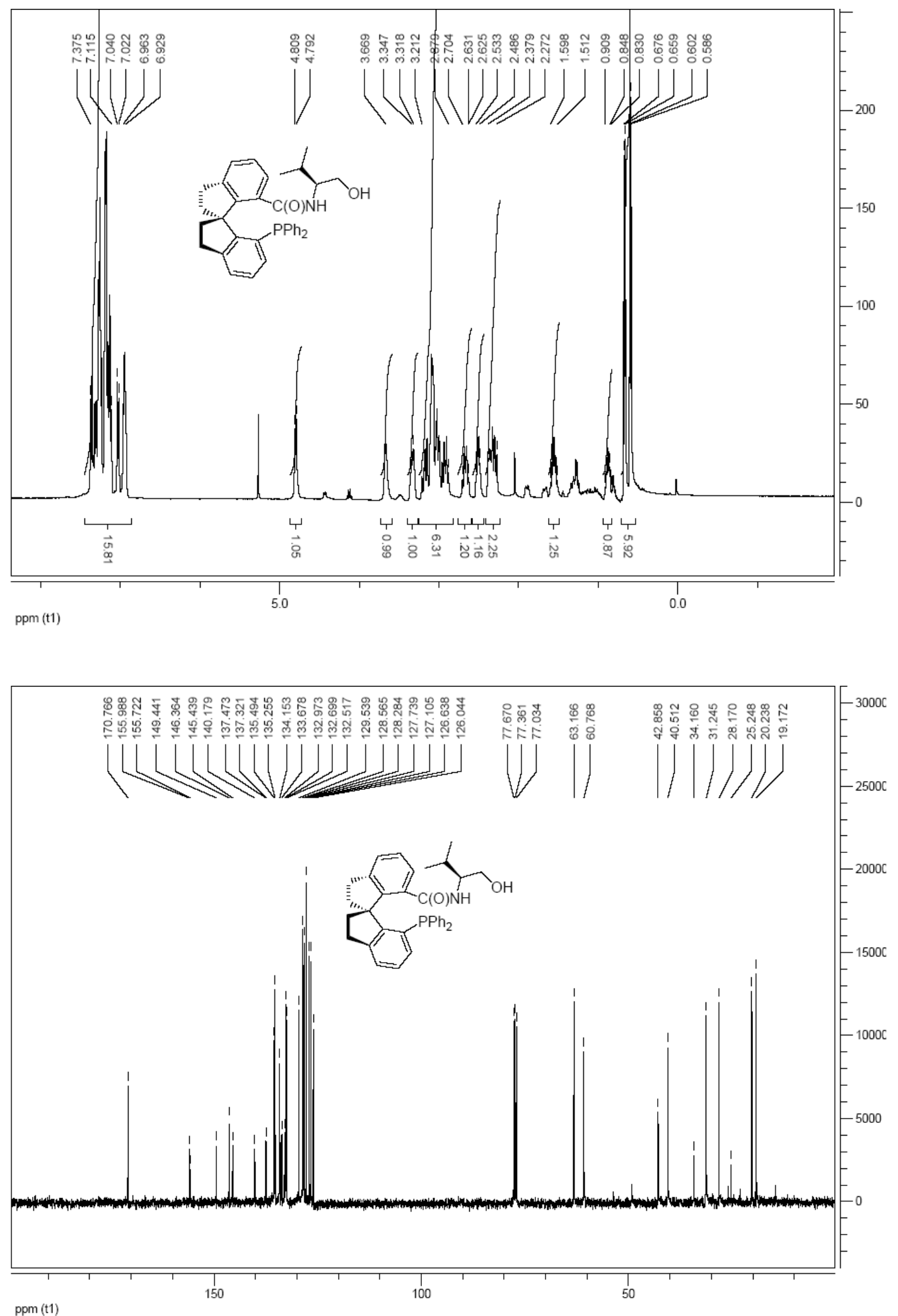
(S,R)-N-(1-Hydroxymethyl-2-methylpropyl)-7'-diphenylphosphino-1,1'-spirobiindane-7carboxylamide $((\boldsymbol{S}, \boldsymbol{R})-\mathbf{6 a})$ : Stiff oil and solidified with standing, $100 \%$ yield. $[\alpha]^{20}{ }_{\mathrm{D}}+178.2$ (c 0.5, $\mathrm{CH}_{2} \mathrm{Cl}_{2}$ ); ${ }^{1} \mathrm{H}$ NMR (300 MHz, $\left.\mathrm{CDCl}_{3}\right) \delta 7.38-7.12$ (m, 13H, Ar-H), 7.00 (d, $J=6.9 \mathrm{~Hz}$, 1H, Ar-H), 6.92-6.87 (m, 2H, Ar-H), 4.55 (d, $J=9.0 \mathrm{~Hz}, 1 \mathrm{H}, \mathrm{NH}), 3.40-3.22\left(\mathrm{~m}, 1 \mathrm{H}, \mathrm{CH}_{2}\right.$ ), 3.22-2.62 (m, 8H, $\mathrm{CH}_{2}$ ), 2.42-2.26 (m, 2H, $\left.\mathrm{CH}_{2}\right), 1.46-1.34$ (m, $\left.1 \mathrm{H}, \mathrm{CH}\right), 0.77$ (d, $J=6.6 \mathrm{~Hz}$, $\left.3 \mathrm{H}, \mathrm{CH}_{3}\right), 0.70$ (d, $\left.J=6.6 \mathrm{~Hz}, 3 \mathrm{H}, \mathrm{CH}_{3}\right) ;{ }^{1} \mathrm{P} \mathrm{NMR}\left(121 \mathrm{MHz}, \mathrm{CDCl}_{3}\right) \delta-20.8(\mathrm{~s}) ;{ }^{13} \mathrm{C} \mathrm{NMR}$ $\left(75 \mathrm{MHz}, \mathrm{CDCl}_{3}\right) \delta 170.3,156.5,149.5,146.6,145.9,140.3,137.3,137.1,135.7,135.4$, 133.7, 133.1, 132.5, 132.2, 129.9, 128.8, 128.7, 128.3, 127.9, 127.1, 126.6, 126.1, 63.5, 63.2, 57.4, 43.1, 43.0, 40.5, 34.2, 31.4, 31.2, 28.9, 25.1, 19.8, 19.3; HRMS (FAB) calcd for $\mathrm{C}_{35} \mathrm{H}_{37} \mathrm{NO}_{2} \mathrm{P}:$ 534.2556; Found 534.2555.

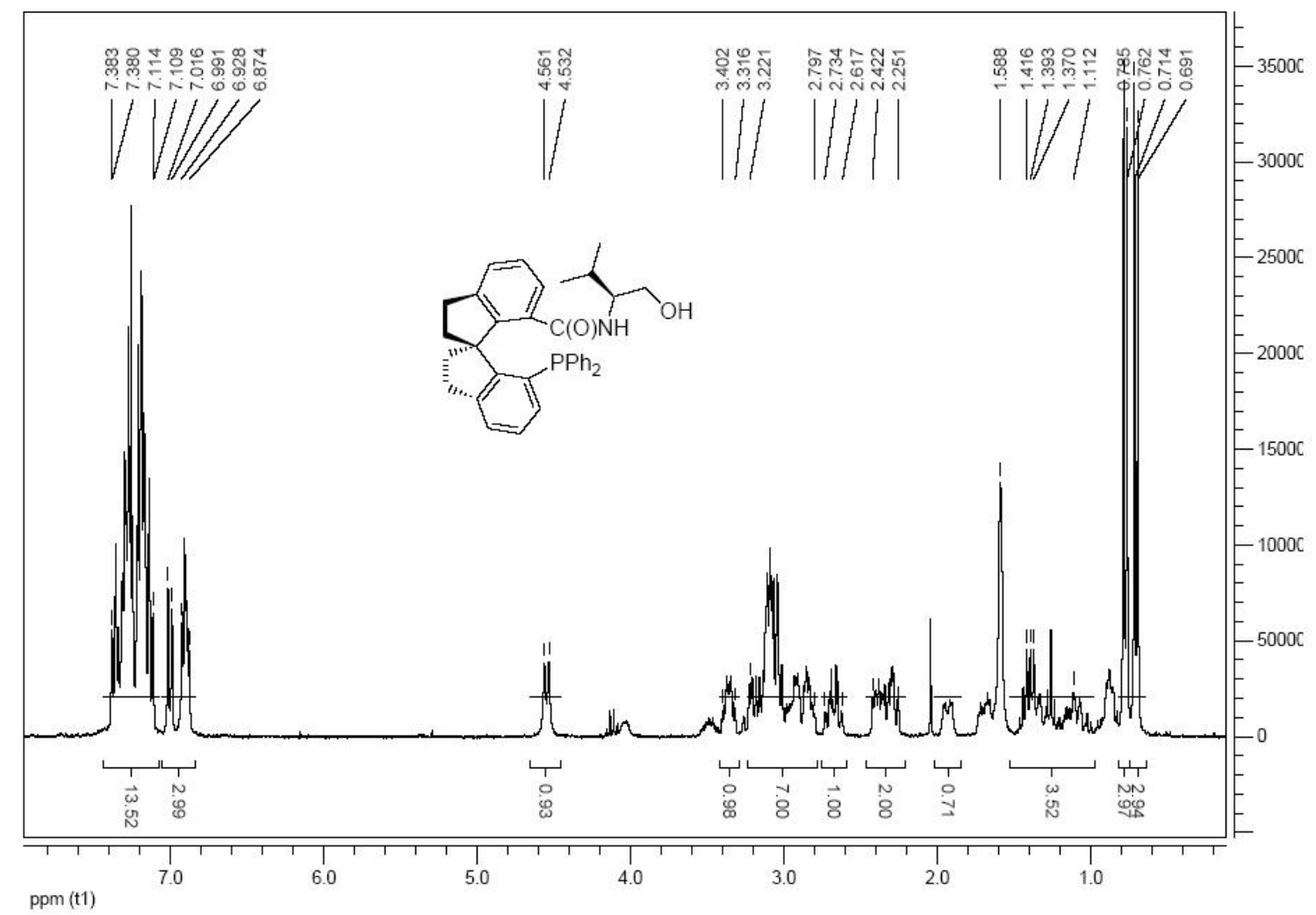




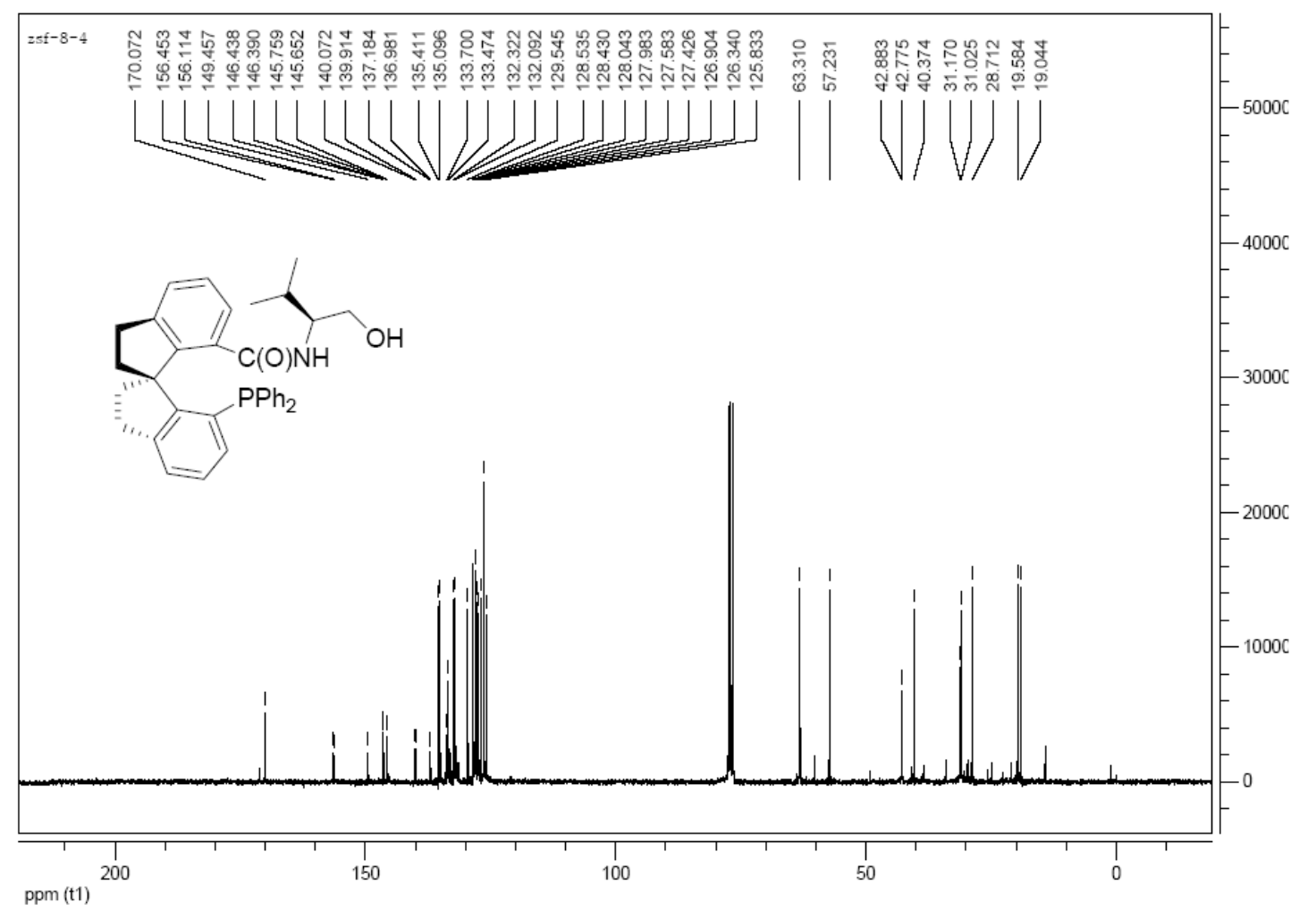

(S,S)-N-(1-Hydroxymethyl-2-methylphenyl)-7'-diphenylphosphino-1,1'-spirobiindane-7carboxylamide $((\boldsymbol{S}, \boldsymbol{S})-\mathbf{6 b})$ : Stiff oil and solidified with standing, $87 \%$ yield. $[\alpha]^{20}{ }_{\mathrm{D}}-169.2(\mathrm{C}$ 0.5, $\mathrm{CH}_{2} \mathrm{Cl}_{2}$ ); ${ }^{1} \mathrm{H}$ NMR (400 MHz, $\mathrm{CDCl}_{3}$ ) $\delta 7.36-6.84$ (m, 21H, Ar-H), 5.17 (d, $J=4.8 \mathrm{~Hz}$, $1 \mathrm{H}, \mathrm{NH}), 4.27$ (s, $1 \mathrm{H}, \mathrm{OH}), 3.65-3.60(\mathrm{~m}, 1 \mathrm{H}, \mathrm{CH}), 3.46-3.37\left(\mathrm{~m}, 2 \mathrm{H}, \mathrm{CH}_{2}\right), 3.11-2.88(\mathrm{~m}$, $\left.4 \mathrm{H}, \mathrm{CH}_{2}\right), 2.85-2.80\left(\mathrm{~m}, 1 \mathrm{H}, \mathrm{CH}_{2}\right), 2.57-2.49\left(\mathrm{~m}, 1 \mathrm{H}, \mathrm{CH}_{2}\right), 2.33-2.24\left(\mathrm{~m}, 2 \mathrm{H}, \mathrm{CH}_{2}\right) ;{ }^{31} \mathrm{P}$ NMR (161 MHz, $\left.\mathrm{CDCl}_{3}\right) \delta-17.7(\mathrm{~s}) ;{ }^{13} \mathrm{C} \mathrm{NMR}\left(100 \mathrm{MHz}, \mathrm{CDCl}_{3}\right) \delta$ 170.6, 155.4, 148.9, 146.5, 139.8, 138.7, 136.7, 134.9, 134.7, 134.0, 133.4, 133.0, 132.8, 132.5, 129.2, 128.9, 128.4, 128.2, 127.9, 127.7, 127.2, 127.0, 126.9, 126.0, 66.5, 63.2, 58.4, 42.3, 40.7, 31.3, 31.2; MS (ESI) m/z 566 (M-1, 100); Anal. calcd for $\mathrm{C}_{38} \mathrm{H}_{34} \mathrm{NO}_{2} \mathrm{P}$ : C 80.40, H 6.04, N 2.47; Found: C 79.17, H 6.09, N 2.50. 

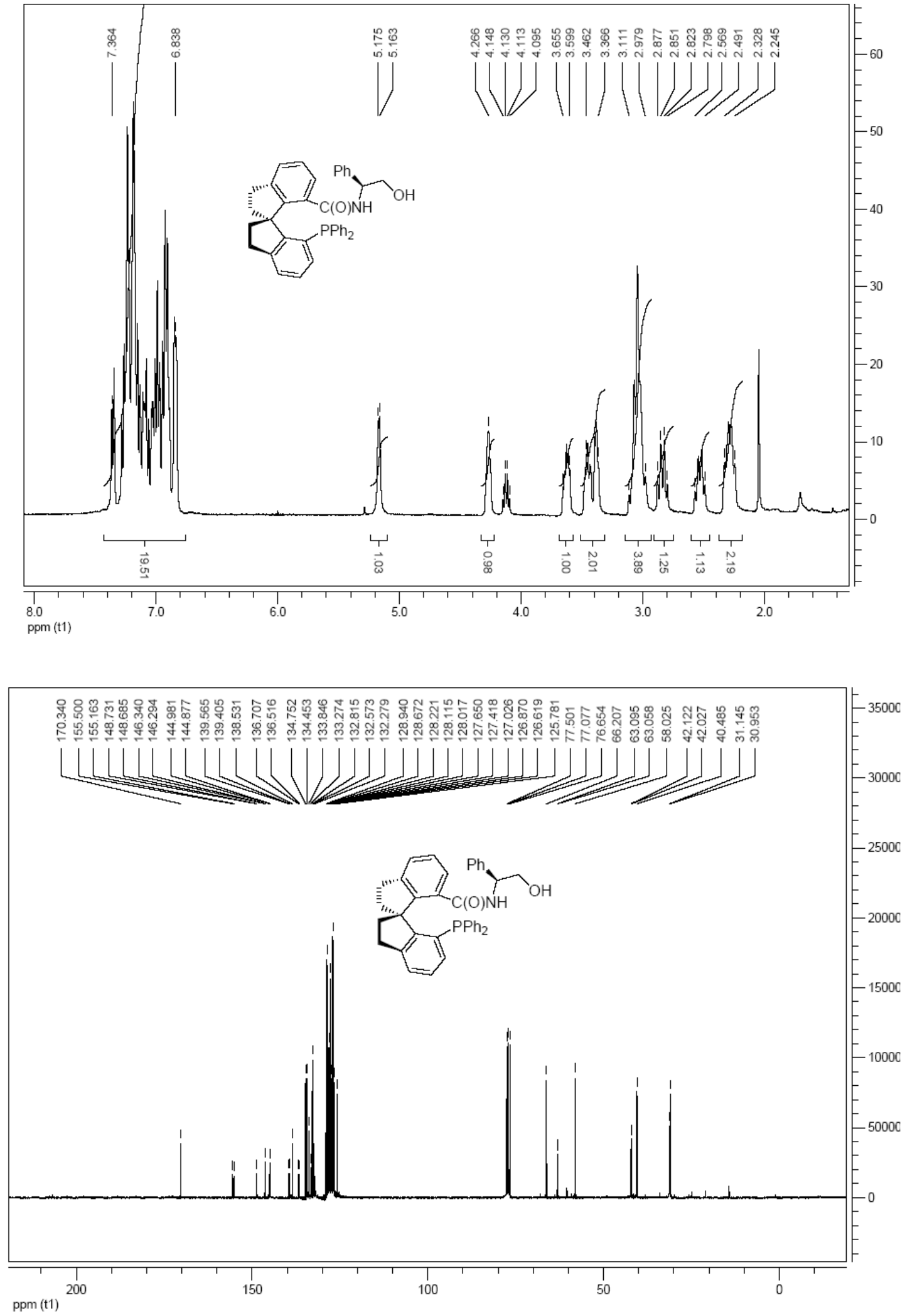

(S,S)-N-(1-Hydroxymethyl-2-methylbenzyl)-7'-diphenylphosphino-1,1'-spirobiindane-7carboxylamide $((\boldsymbol{S}, \boldsymbol{S})-6 \mathrm{c})$ : White solid, 95\% yield. Mp $178-180{ }^{\circ} \mathrm{C}$; $[\alpha]^{20}{ }_{\mathrm{D}}-242.2$ (c 0.5 , $\mathrm{CH}_{2} \mathrm{Cl}_{2}$ ); ${ }^{1} \mathrm{H}$ NMR (400 MHz, $\left.\mathrm{CDCl}_{3}\right) \delta 7.36-6.80(\mathrm{~m}, 21 \mathrm{H}, \mathrm{Ar}-\mathrm{H}), 4.68$ (br, $\left.1 \mathrm{H}, \mathrm{NH}\right)$, 3.21-2.59 (m, 8H, $\mathrm{CH}_{2}$ and $\mathrm{CH}$ ), 2.69-2.61 (m, 1H, $\left.\mathrm{CH}_{2}\right), 2.39-2.18\left(\mathrm{~m}, 4 \mathrm{H}, \mathrm{CH}_{2}\right) ;{ }^{31} \mathrm{P} \mathrm{NMR}$ $\left(161 \mathrm{MHz}, \mathrm{CDCl}_{3}\right) \delta-20.8(\mathrm{~s}) ;{ }^{13} \mathrm{C} \mathrm{NMR}\left(100 \mathrm{MHz}, \mathrm{CDCl}_{3}\right) \delta 170.3,156.0,155.8,149.2$, 146.2, 145.6, 140.1, 138.7, 137.3, 137.1, 135.5, 134.1, 133.6, 133.2, 133.0, 132.7, 132.5, 
129.6, 129.4, 128.7, 128.3, 127.8, 127.8, 127.1, 126.7, 126.6, 126.1, 63.6, 63.2, 55.4, 42.6, 40.7, 35.9, 31.4, 31.2; HRMS (FAB) calcd for $\mathrm{C}_{39} \mathrm{H}_{35} \mathrm{NO}_{2} \mathrm{P}: 580.2400$; Found 580.2385.
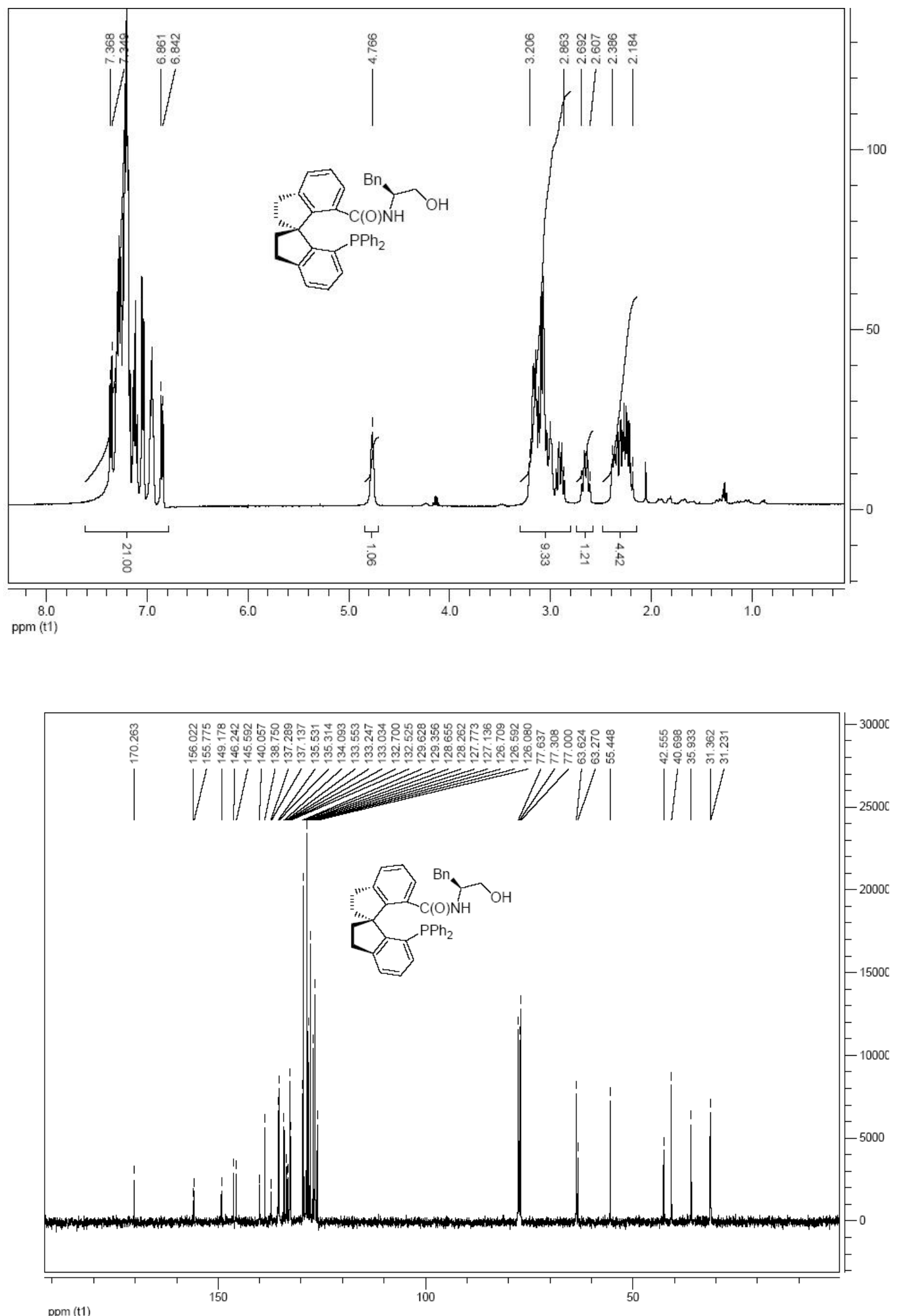

(S,S)-N-(1-Hydroxymethyl-2-methylbenzyl)-7'-di(4-methoxyphenyl)phosphino-1,1'-spiro biindane-7-carboxylamide ((S,S)-6d): White solid, 93\% yield. Mp 148-150 ${ }^{\circ} \mathrm{C} ;[\alpha]^{19}{ }_{\mathrm{D}}$ 
-212.6 (c 0.5, $\mathrm{CH}_{2} \mathrm{Cl}_{2}$ ); ${ }^{1} \mathrm{H}$ NMR (300 MHz, $\mathrm{CDCl}_{3}$ ) $\delta$ 7.34-6.99 (m, 12H, Ar-H), 6.89-6.72 (m, 7H, Ar-H), 4.91 (d, $J=6.9 \mathrm{~Hz}, 1 \mathrm{H}, \mathrm{CH}), 3.76$ (s, 3H, $\mathrm{CH}_{3}$ ), 3.75 (s, 3H, $\mathrm{CH}_{3}$ ), 3.23-2.49 (m, 10H, $\mathrm{CH}_{2}$ ), 2.32-2.16(m, 4H, $\left.\mathrm{CH}_{2}\right)$; ${ }^{31} \mathrm{P}$ NMR (121 MHz, $\left.\mathrm{CDCl}_{3}\right) \delta-23.8(\mathrm{~s}) ;{ }^{13} \mathrm{C} \mathrm{NMR}$ $\left(75 \mathrm{MHz} \mathrm{CDCl}_{3}\right) \delta 170.2,160.7,159.4,155.3,155.0,148.5,146.0,145.2,138.5,136.7$, 136.3, 134.2, 134.0, 133.8, 133.6, 133.4, 130.6, 130.4, 130.3, 129.0, 128.4, 127.9, 127.7, 127.4, 127.0, 126.5, 126.3, 125.6, 114.0, 113.9, 113.8, 63.5, 63.1, 55.2, 55.1, 49.3, 42.0, 41.9, 40.5, 35.7, 33.9, 31.1, 30.9, 25.6, 24.9; HRMS (EI) calcd for $\mathrm{C}_{41} \mathrm{H}_{40} \mathrm{NO}_{4} \mathrm{P}:$ 614.2695; Found 614.2698 .

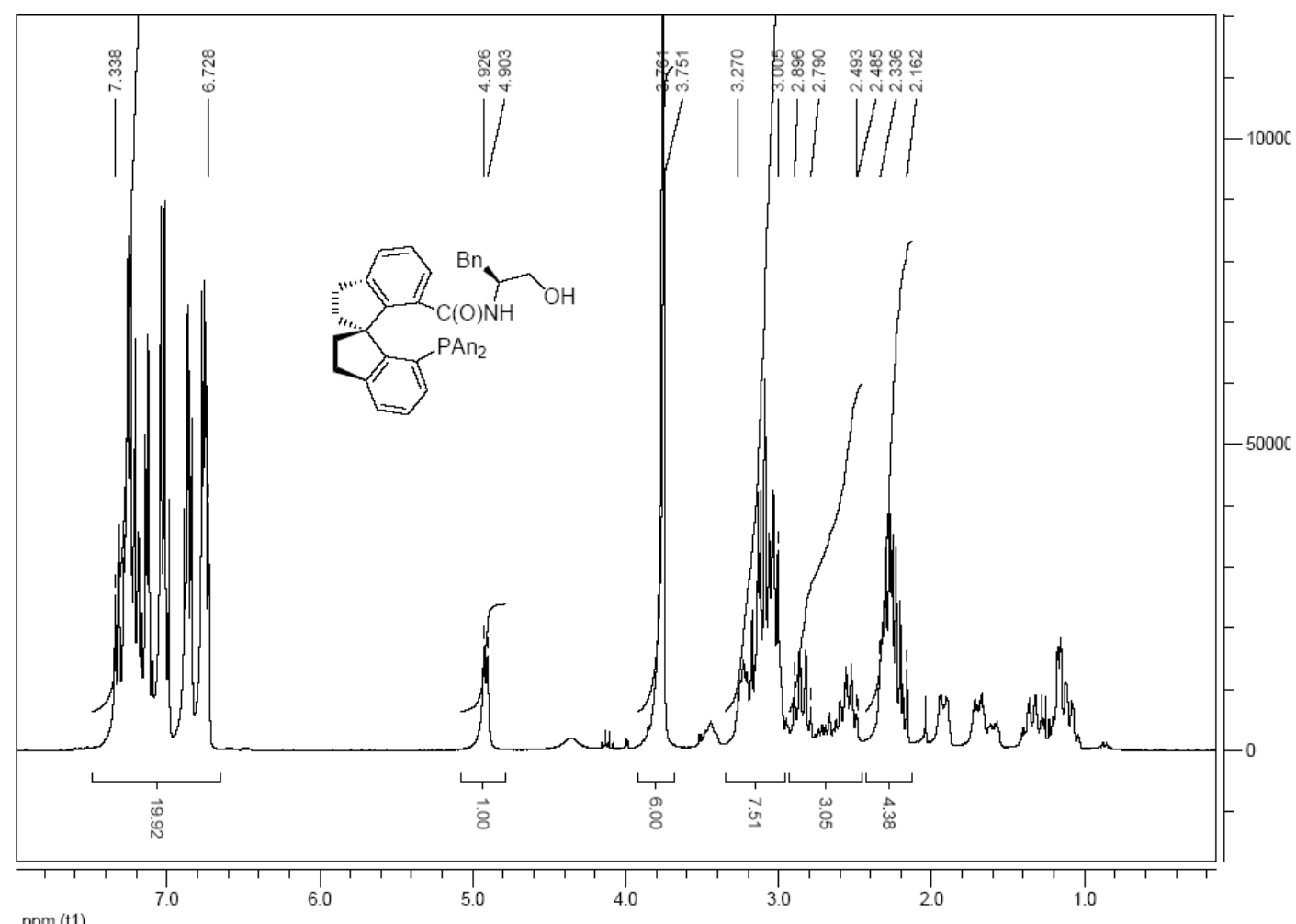




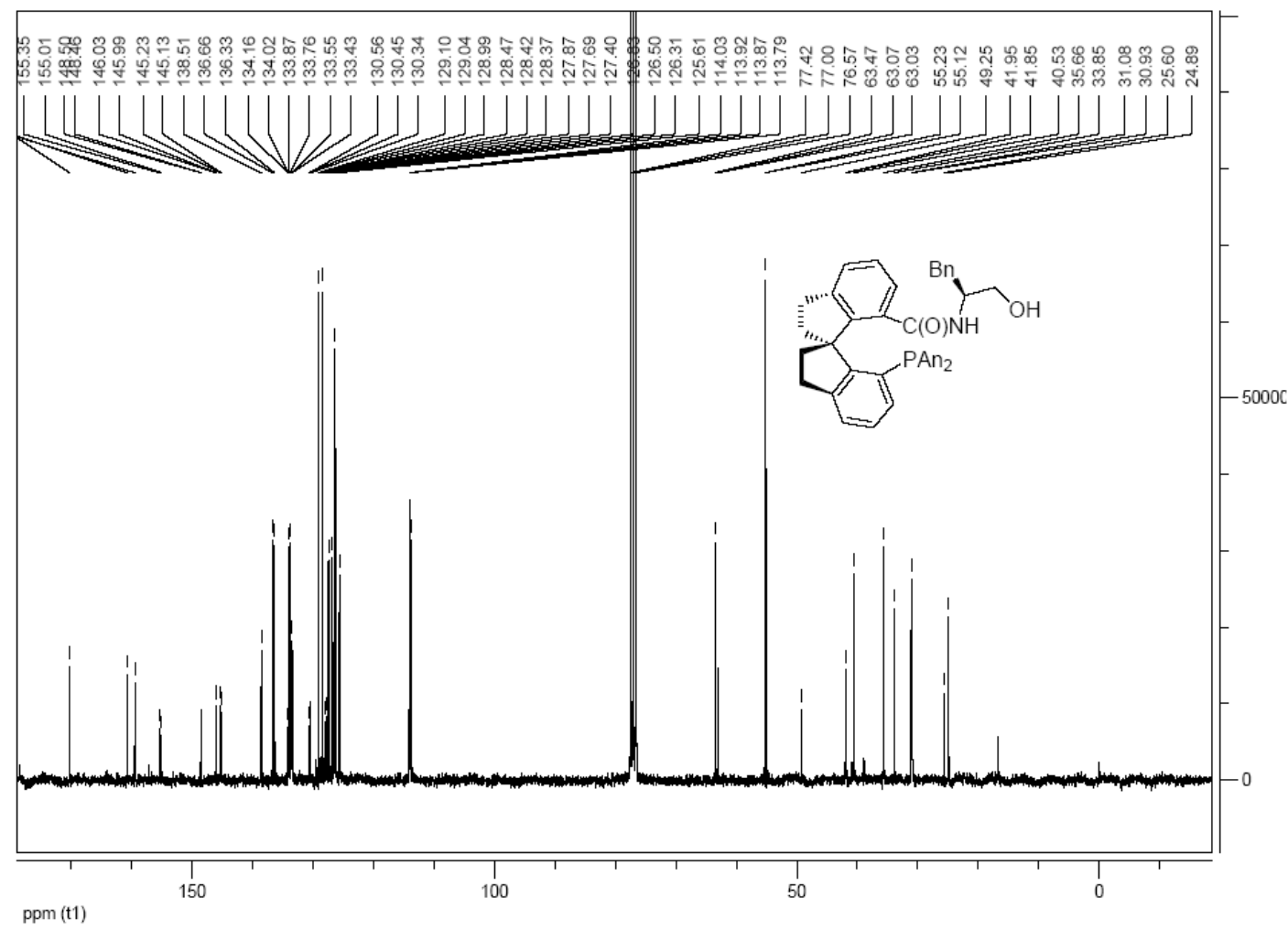

(S,S)-N-(1-Hydroxymethyl-2-methylbenzyl)-7'-di(3,5-dimethylphenyl)phosphino-1,1'-spi robiindane-7-carboxylamide $((\boldsymbol{S}, \boldsymbol{S})-\mathbf{6 e})$ : Stiff oil and solidified with standing, $91 \%$ yield. $[\alpha]^{17}{ }_{\mathrm{D}}-253.4$ (c 0.5, $\mathrm{CH}_{2} \mathrm{Cl}_{2}$ ); ${ }^{1} \mathrm{H}$ NMR (300 MHz, $\mathrm{CDCl}_{3}$ ) $\delta$ 7.36-6.99 (m, 10H, Ar-H), 6.82 (d, $J=11.4 \mathrm{~Hz}, 2 \mathrm{H}, \mathrm{Ar}-\mathrm{H}), 6.67$ (d, $J=7.8 \mathrm{~Hz}, 3 \mathrm{H}, \mathrm{Ar}-\mathrm{H}), 6.52$ (d, $J=6.9 \mathrm{~Hz}, 2 \mathrm{H}, \mathrm{Ar}-\mathrm{H})$, 4.23 (d, $J=6.6 \mathrm{~Hz}, 1 \mathrm{H}, \mathrm{CH}), 3.22-2.80\left(\mathrm{~m}, 9 \mathrm{H}, \mathrm{CH}_{2}\right), 2.44-2.26\left(\mathrm{~m}, 5 \mathrm{H}, \mathrm{CH}_{2}\right), 2.16(\mathrm{~s}, 6 \mathrm{H}$, $\mathrm{CH}_{3}$ ), 2.15 (s, 6H, CH3); ${ }^{31} \mathrm{P}$ NMR (121 MHz, $\left.\mathrm{CDCl}_{3}\right) \delta-20.8(\mathrm{~s}) ;{ }^{13} \mathrm{C}$ NMR $(75 \mathrm{MHz}$, $\left.\mathrm{CDCl}_{3}\right) \delta 170.8,156.0,155.7,149.7,146.3,145.7,145.5,140.5,140.3,139.0,137.9,137.8$, 137.6, 136.8, 136.6, 134.2, 133.8, 133.5, 131.7, 130.8, 129.8, 129.6, 129.3, 128.7, 127.8, 127.4, 126.7, 126.5, 126.0, 125.1, 64.1, 63.2, 56.4, 43.3, 43.2, 41.2, 41.0, 35.5, 34.0, 31.4, 31.2, 21.5, 21.4; HRMS (EI) calcd for $\mathrm{C}_{43} \mathrm{H}_{44} \mathrm{NO}_{2} \mathrm{P}$ : 637.3109; Found 637.3105. 


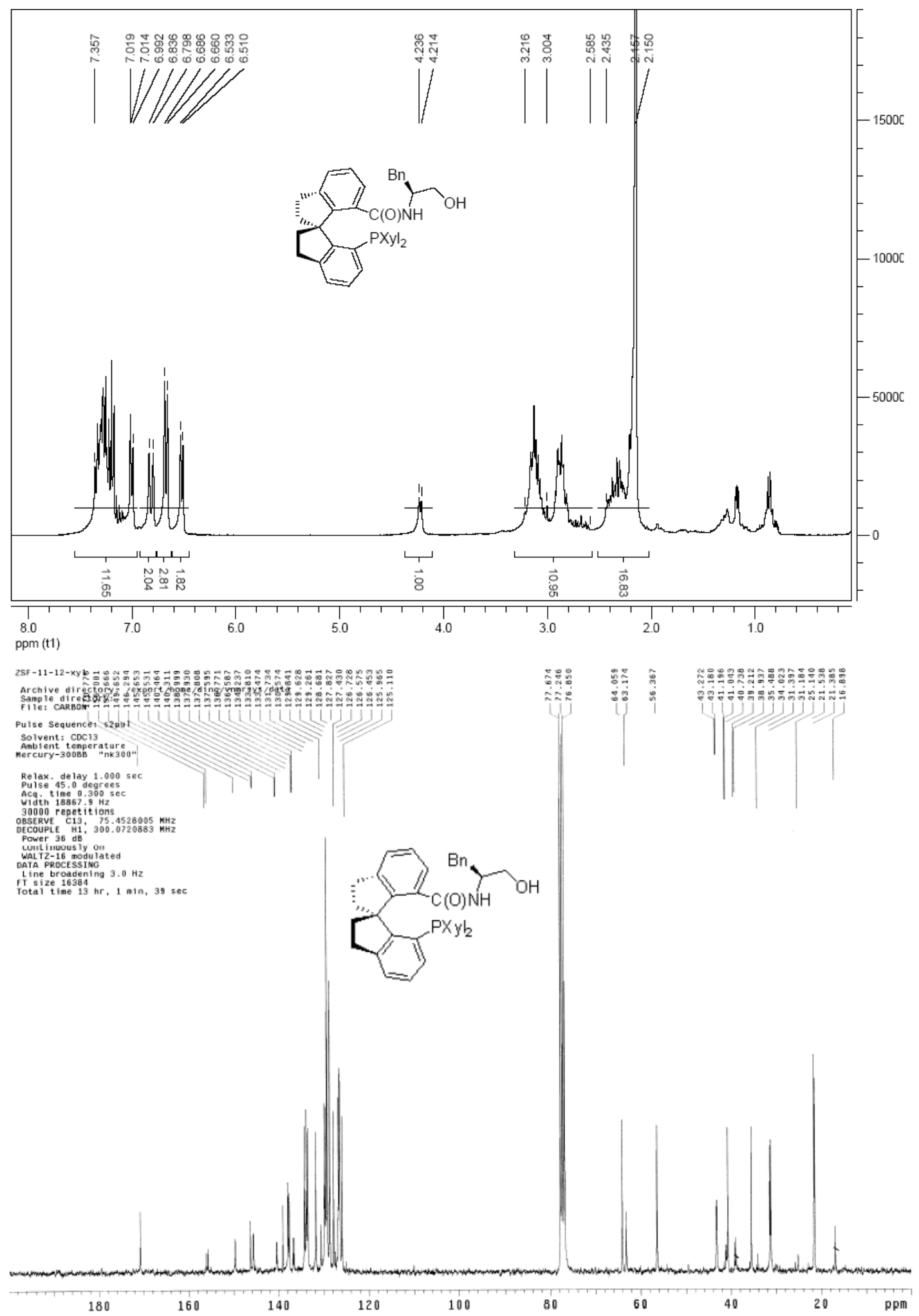

(S,S)-7-[4-(Isopropyl)oxazol-2-yl]-7'-diphenylphosphinyl-1,1'-spirobiinane

$((S, S)-7 a):$

White foamy solid, 69\% yield. Mp 40-45 ${ }^{\circ} \mathrm{C}$; $[\alpha]^{20}{ }_{\mathrm{D}}-243.2\left(c \mathrm{cos}, \mathrm{CH}_{2} \mathrm{Cl}_{2}\right) ;{ }^{1} \mathrm{H}$ NMR (300 $\left.\left.\mathrm{MHz}_{\mathrm{CDCl}}\right)\right) \mathrm{\delta} 7.54$ (d, $\left.J=6.6 \mathrm{~Hz}, 1 \mathrm{H}, \mathrm{Ar}-\mathrm{H}\right), 7.26-6.89(\mathrm{~m}, 14 \mathrm{H}, \mathrm{Ar}-\mathrm{H}), 6.76-6.72(\mathrm{~m}, 1 \mathrm{H}$, Ar-H), 3.46-3.37 (m, 2H, CH $)$, 3.17-3.09 (m, 1H, CH), 2.98-2.82 (m, 3H, $\left.\mathrm{CH}_{2}\right), 2.72-2.52$ (m, 2H, $\mathrm{CH}_{2}$ ), 2.18-2.11 (m, 1H, $\left.\mathrm{CH}_{2}\right), 2.10-2.01$ (m, 1H, $\left.\mathrm{CH}_{2}\right), 1.80-1.74\left(\mathrm{~m}, 1 \mathrm{H}, \mathrm{CH}_{2}\right)$, 1.36-1.19 (m, 1H, CH), 0.80 (d, J = 6.6 Hz, 3H, $\left.\mathrm{CH}_{3}\right), 0.67$ (d, J = 6.6 Hz, 3H, CH ${ }_{3}$ ); ${ }^{1} \mathrm{P}$ NMR (121 MHz, $\left.\left.\mathrm{CDCl}_{3}\right) \delta-20.3(\mathrm{~s}) ;{ }^{13} \mathrm{C} \mathrm{NMR} \mathrm{(75} \mathrm{MHz,} \mathrm{CDCl}_{3}\right) \delta 164.3,154.7,154.3$, 
149.2, 145.1, 144.9, 144.8, 138.8, 138.6, 138.5, 138.3, 134.2, 133.9, 133.5, 132.7, 132.4, 132.0, 128.8, 128.4, 128.1, 128.0, 126.9, 126.8, 126.0, 125.1, 72.3, 68.6, 63.5, 40.3, 38.6, 32.9, 31.2, 30.8, 29.9, 19.4, 18.4; HRMS (FAB) calcd for $\mathrm{C}_{35} \mathrm{H}_{34} \mathrm{NOP}$ : 516.2451; Found 516.2454 .
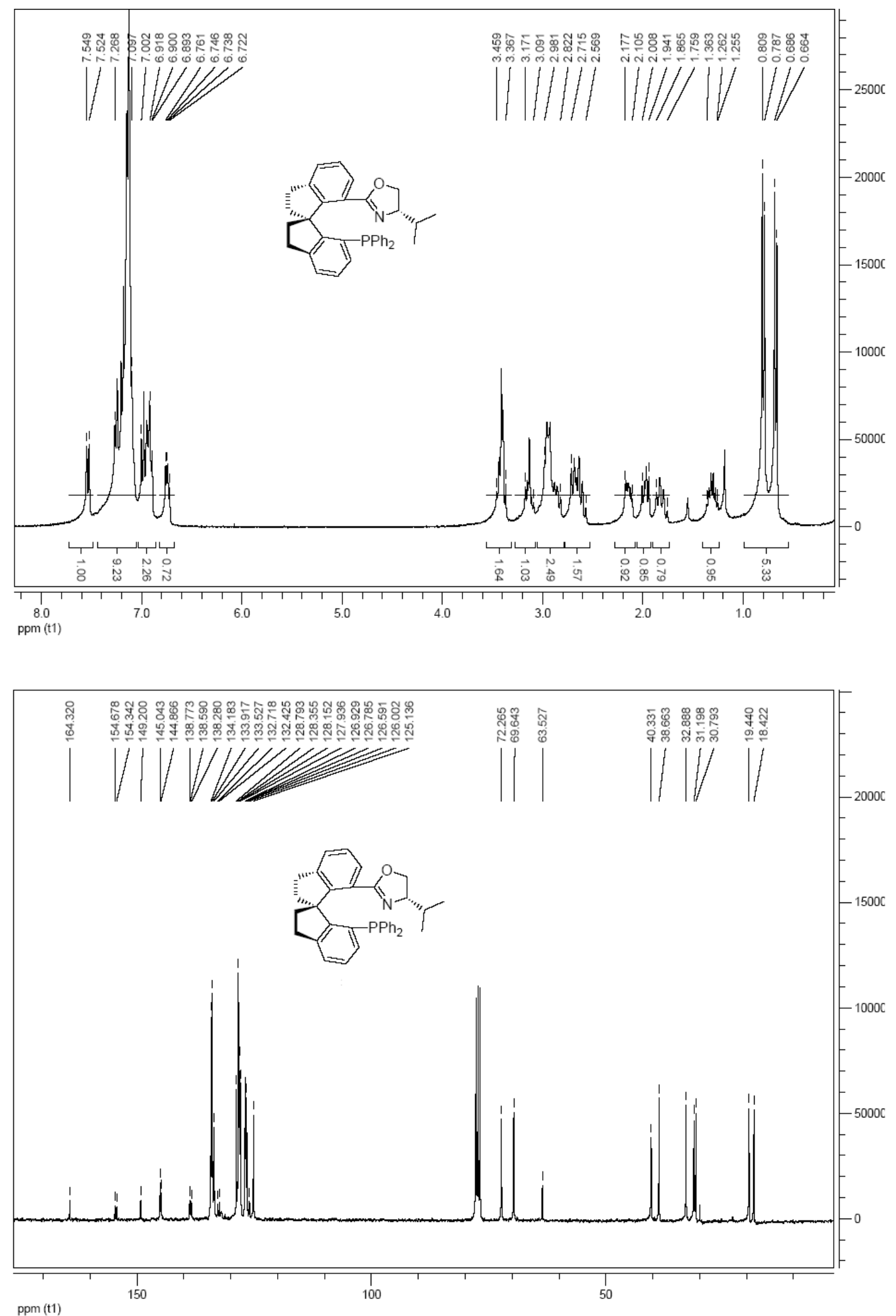
Stiff oil and solidified with standing, 89\% yield. $\left.[\alpha]^{20}{ }_{\mathrm{D}}+193.4(c) .5, \mathrm{CH}_{2} \mathrm{Cl}_{2}\right) ;{ }^{1} \mathrm{H}$ NMR (300 $\left.\mathrm{MHz} \mathrm{CDCl}_{3}\right) \delta 7.40$ (d, $\left.J=7.5 \mathrm{~Hz}, 1 \mathrm{H}, \mathrm{Ar}-\mathrm{H}\right), 7.35-6.94$ (m, 14, Ar-H), 6.89-6.82 (m, 1H, Ar-H), 3.79 (t, $J=7.5 \mathrm{~Hz}, 1 \mathrm{H}, \mathrm{CH}) ; 3.22-2.75\left(\mathrm{~m}, 7 \mathrm{H}, \mathrm{CH}_{2}\right), 2.25-2.12\left(\mathrm{~m}, 3 \mathrm{H}, \mathrm{CH}_{2}\right)$, 1.30-1.18 (m, 1H, CH), 0.88 (d, $\left.J=6.3 \mathrm{~Hz}, 3 \mathrm{H}, \mathrm{CH}_{3}\right), 0.63$ (d, $\left.J=6.6 \mathrm{~Hz}, 3 \mathrm{H}, \mathrm{CH}_{3}\right) ;{ }^{31} \mathrm{P}$ NMR (121 MHz, $\left.\mathrm{CDCl}_{3}\right) \delta-21.1$ (s); $\left.{ }^{13} \mathrm{C} \mathrm{NMR} \mathrm{(75} \mathrm{MHz,} \mathrm{CDCl}_{3}\right) \delta 162.9,155.7,155.3$, 150.7, 145.3, 145.2, 145.1, 139.4, 139.2, 138.1, 137.9, 134.4, 134.1, 133.8, 133.7, 133.5, 131.9, 131.7, 128.8, 128.3, 128.2, 128.1, 126.9, 126.7, 126.4, 125.4, 125.0, 73.7, 70.1, 63.6, 41.5, 38.2, 33.1, 31.4, 30.9, 20.1, 19.2; MS (ESI) m/z $516\left(\mathrm{M}^{+}+1,100\right)$; Anal. calcd for $\mathrm{C}_{35} \mathrm{H}_{35} \mathrm{NOP}$ : C 81.53, H 6.65; Found: C 81.42, H 6.50.

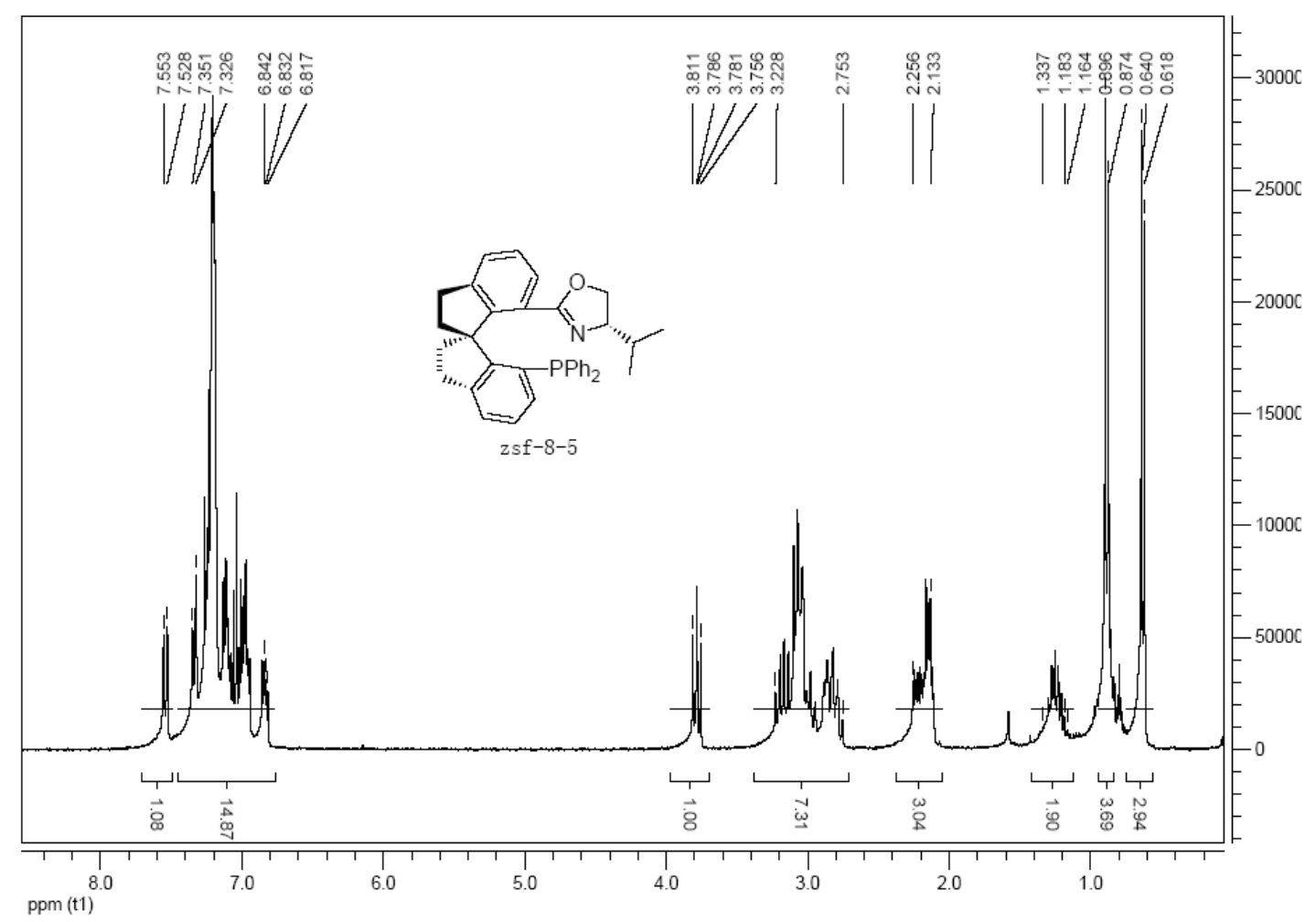




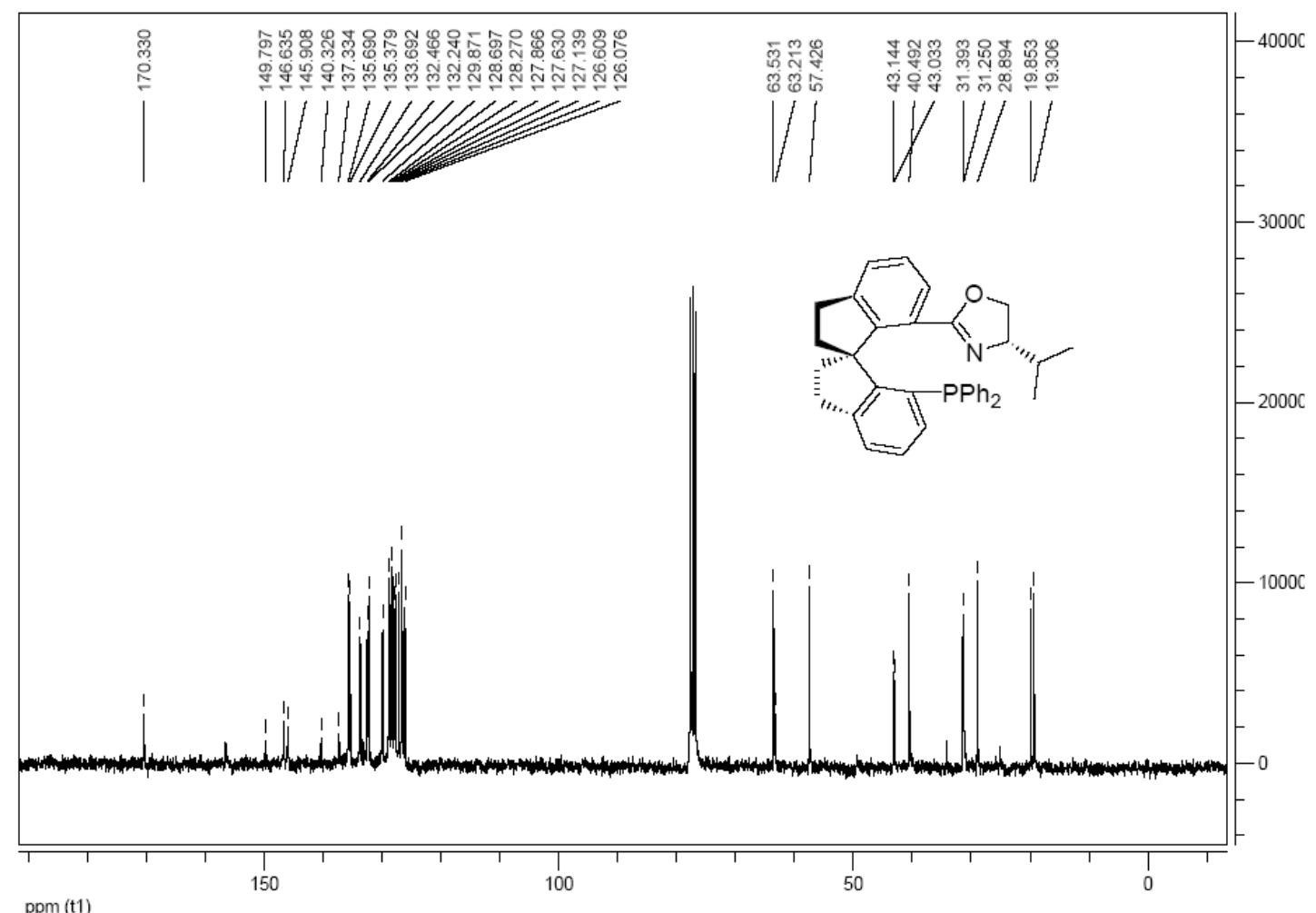

(S,S)-7-[4-(Phenyl)oxazol-2-yl]-7'-diphenylphosphinyl-1,1'-spirobiinane ((S,S)-7b): White solid, 69\% yield. Mp 159-160 ${ }^{\circ} \mathrm{C}$; $[\alpha]^{20}{ }_{\mathrm{D}}-238.0$ (c 0.5, $\left.\mathrm{CH}_{2} \mathrm{Cl}_{2}\right)$; ${ }^{1} \mathrm{H}$ NMR (300 MHz, $\mathrm{CDCl}_{3}$ ) $\delta 7.77$ (d, $J=7.5 \mathrm{~Hz}, 1 \mathrm{H}, \mathrm{Ar}-\mathrm{H}), 7.40-6.85$ (m, 20H, Ar-H), 4.78 (dd, $J=10.2$ and $6.9 \mathrm{~Hz}$, $1 \mathrm{H}, \mathrm{CH}$ ), 3.67 (dd, $J=8.1$ and $7.2 \mathrm{~Hz}, 1 \mathrm{H}, \mathrm{CH}_{2}$ ), 3.37 (t, $\left.J=8.4 \mathrm{~Hz}, 1 \mathrm{H}, \mathrm{CH}_{2}\right), 3.12-2.94(\mathrm{~m}$, 3H, $\mathrm{CH}_{2}$ ), 2.86-2.78 (m, 1H, $\mathrm{CH}_{2}$ ), 2.65 (dd, $J=22.2$ and $\left.10.5 \mathrm{~Hz}, 1 \mathrm{H}, \mathrm{CH}_{2}\right), 2.29-1.95(\mathrm{~m}$, $\left.3 \mathrm{H}, \mathrm{CH}_{2}\right) ;{ }^{31} \mathrm{P}$ NMR $\left(121 \mathrm{MHz}, \mathrm{CDCl}_{3}\right) \delta-20.6(\mathrm{~s}) ;{ }^{13} \mathrm{C} \mathrm{NMR}\left(75 \mathrm{MHz}, \mathrm{CDCl}_{3}\right) \delta 165.6$, 154.9, 154.6, 149.6, 145.1, 144.7, 144.6, 142.7, 138.8, 138.6, 137.9, 137.7, 134.2, 134.1, 133.9, 133.8, 133.6, 133.5, 132.4, 129.2, 128.5, 128.2, 128.1, 128.0, 127.9, 127.2, 126.7, 126.6, 126.5, 73.8, 69.1, 63.6, 40.7, 40.6, 38.4, 31.0, 30.7; MS (EI) m/z 549 (M, 54), 402 (100); Anal. calcd for $\mathrm{C}_{38} \mathrm{H}_{32} \mathrm{NOP}$ : C 83.04, H 5.87, N 2.55; Found: C 82.92, H 5.81, N 2.53. 

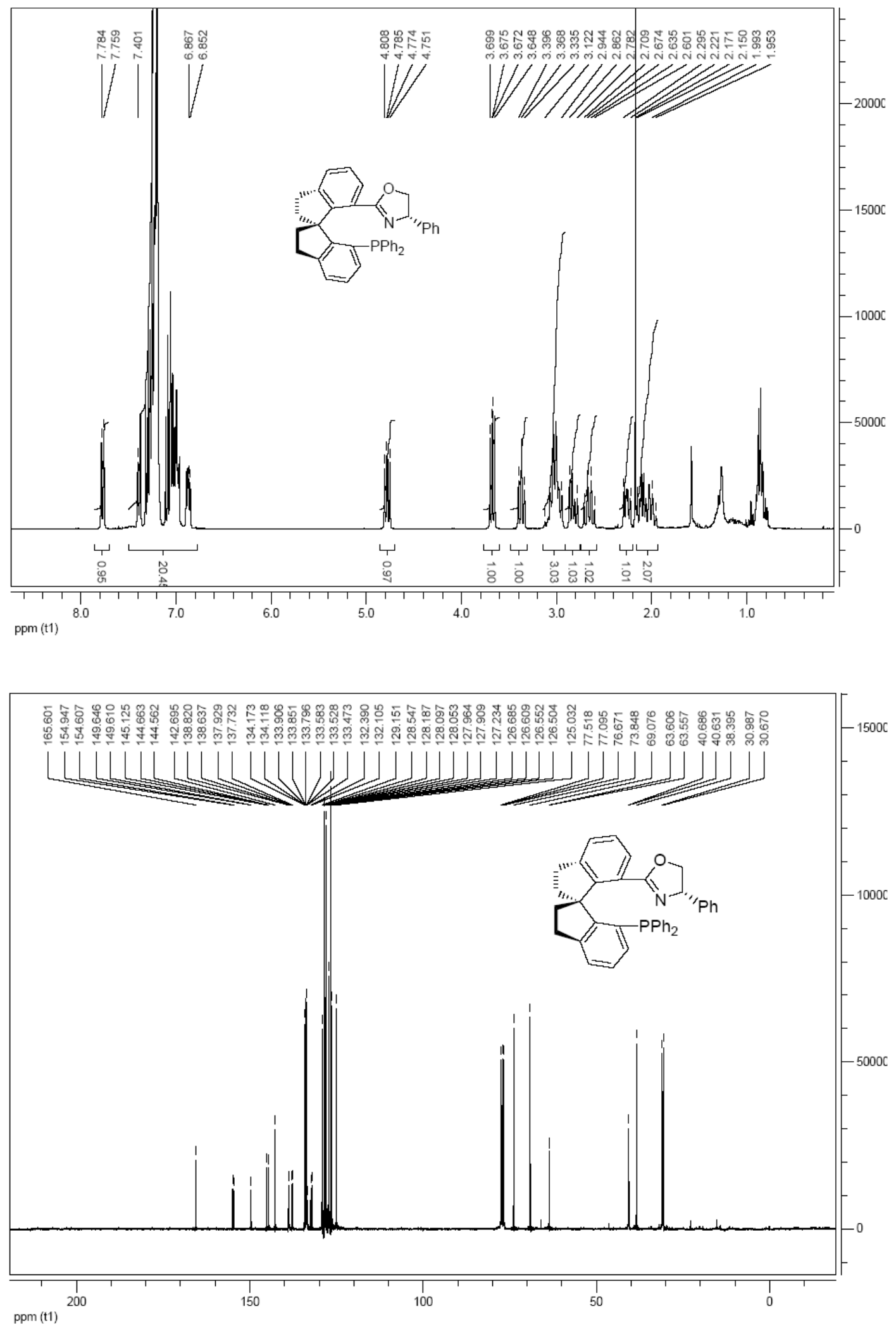

(S,S)-7-[4-(Benzyl)oxazol-2-yl]-7'-diphenylphosphinyl-1,1'-spirobiinane ((S,S)-7c): White foamy solid, 75\% yield. Mp 164-166 ${ }^{\circ} \mathrm{C}$; $[\alpha]^{20}{ }_{\mathrm{D}}-227.0$ (c 0.5, $\mathrm{CH}_{2} \mathrm{Cl}_{2}$ ); ${ }^{1} \mathrm{H}$ NMR (300 MHz, $\left.\mathrm{CDCl}_{3}\right) \delta 7.61$ (d, $\left.J=7.2 \mathrm{~Hz}, 1 \mathrm{H}, \mathrm{Ar}-\mathrm{H}\right), 7.37-6.83$ (m, 20H, Ar-H), 4.11-4.01 (m, 1H, CH), 3.50 (dd, $J=8.4$ and $\left.6.6 \mathrm{~Hz}, 1 \mathrm{H}, \mathrm{CH}_{2}\right), 3.25\left(t, \mathrm{~J}=9.0 \mathrm{~Hz}, 1 \mathrm{H}, \mathrm{CH}_{2}\right), 3.05-2.92(\mathrm{~m}, 3 \mathrm{H}$, $\mathrm{CH}_{2}$ ), 2.82-2.68 (m, 3H, CH 2$), 2.26-1.89\left(\mathrm{~m}, 4 \mathrm{H}, \mathrm{CH}_{2}\right) ;{ }^{31} \mathrm{P}$ NMR (121 $\left.\mathrm{MHz}, \mathrm{CDCl}_{3}\right) \delta$ -20.9 (s); ${ }^{13} \mathrm{C}$ NMR (75 MHz, $\left.\mathrm{CDCl}_{3}\right) \delta 164.3,155.3,155.0,149.7,145.3,145.0,139.0$, 
138.8, 134.3, 134.0, 133.8, 132.5, 132.3, 129.4, 128.9, 128.7, 128.3, 127.2, 126.8, 126.7, 126.5, 125.7, 125.3, 70.9, 67.5, 63.7, 41.3, 40.7, 38.5, 31.2, 30.9; HRMS (FAB) calcd for $\mathrm{C}_{39} \mathrm{H}_{35} \mathrm{NOP}: 564.2451$; Found 564.2463.
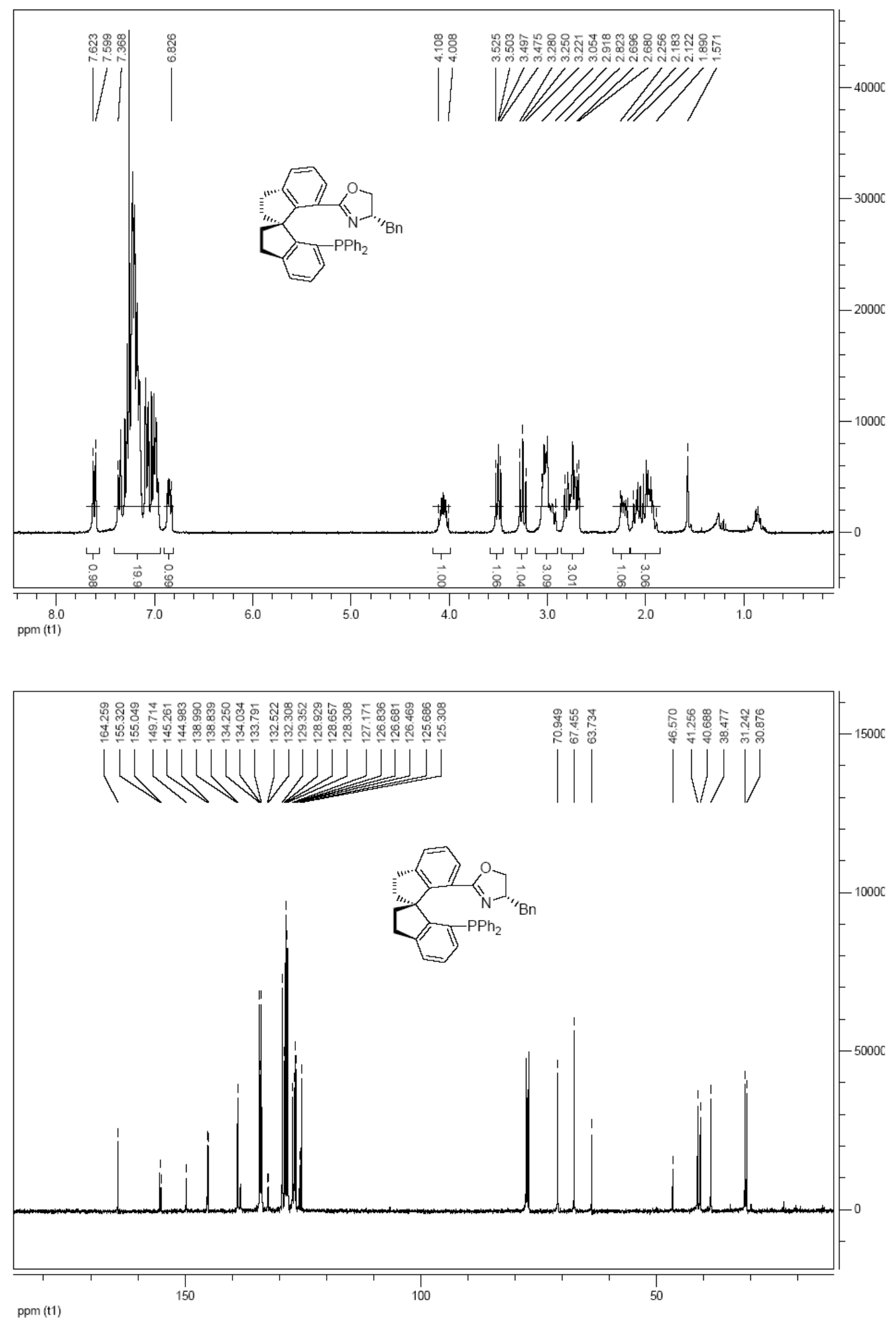

(S,S)-7-[4-(Benzyl)oxazol-2-yl]-7'-di(4-methoxyphenyl)phosphinyl-1,1'-spirobiinane $((\boldsymbol{S}, \boldsymbol{S})-7 \mathrm{~d})$ : Colorless oil and solidified with standing, $76 \%$ yield. $[\alpha]^{20}-221.6$ (c 0.5 , 
$\mathrm{CH}_{2} \mathrm{Cl}_{2}$ ); ${ }^{1} \mathrm{H}$ NMR (300 MHz, $\left.\mathrm{CDCl}_{3}\right) \delta 7.62$ (d, $\left.J=7.2 \mathrm{~Hz}, 1 \mathrm{H}, \mathrm{Ar}-\mathrm{H}\right), 7.36-6.74(\mathrm{~m}, 18 \mathrm{H}$, Ar-H), 4.09-3.99 (m, 1H, $\mathrm{CH}_{2}$ ), 3.77 (s, 3H, $\mathrm{CH}_{3}$ ), 3.76 (s, 3H, $\mathrm{CH}_{3}$ ), 3.50 (dd, $J=8.4$ and $\left.6.6 \mathrm{~Hz}, 1 \mathrm{H}, \mathrm{CH}_{2}\right), 3.21$ (t, $\left.J=9.0 \mathrm{~Hz}, 1 \mathrm{H}, \mathrm{CH}\right) 3.03-2.90\left(\mathrm{~m}, 3 \mathrm{H}, \mathrm{CH}_{2}\right), 2.79-2.66$ (m, 3H, $\mathrm{CH}_{3}$ ), 2.24-2.17 (m, 1H, $\mathrm{CH}_{2}$ ), 2.06-1.98 (m, 2H, $\left.\mathrm{CH}_{2}\right), 1.92-1.81$ (m, $\left.1 \mathrm{H}, \mathrm{CH}_{2}\right)$; ${ }^{31} \mathrm{P}$ NMR $\left(161 \mathrm{MHz}, \mathrm{CDCl}_{3}\right) \delta-23.3(\mathrm{~s}) ;{ }^{13} \mathrm{C} \mathrm{NMR}\left(100 \mathrm{MHz}, \mathrm{CDCl}_{3}\right) \delta 164.4,160.9,159.9,159.8$, 159.7, 159.2, 154.4, 154.2, 149.5, 145.2, 144.8, 138.8, 135.5, 135.3, 133.8, 133.6, 133.1, 129.7, 129.5, 129.3, 128.7, 128.6, 127.0, 126.7, 126.5, 125.9, 125.0, 114.0, 70.9, 67.3, 63.6, 55.3, 41.2, 40.1, 38.5, 31.1, 30.8; HRMS (EI) calcd for $\mathrm{C}_{41} \mathrm{H}_{38} \mathrm{NO}_{3} \mathrm{P}$ : 623.2597; Found 623.2589 .

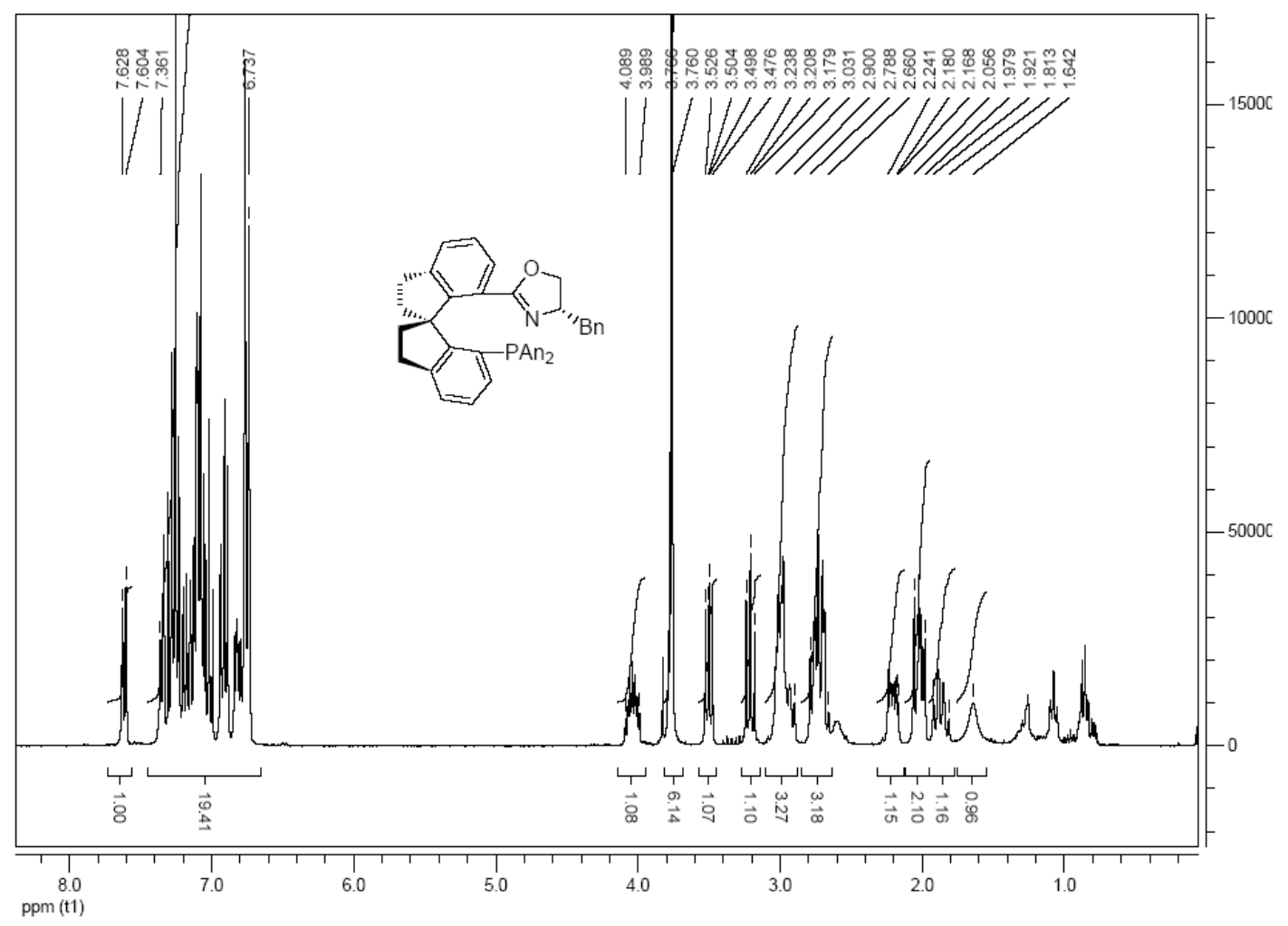




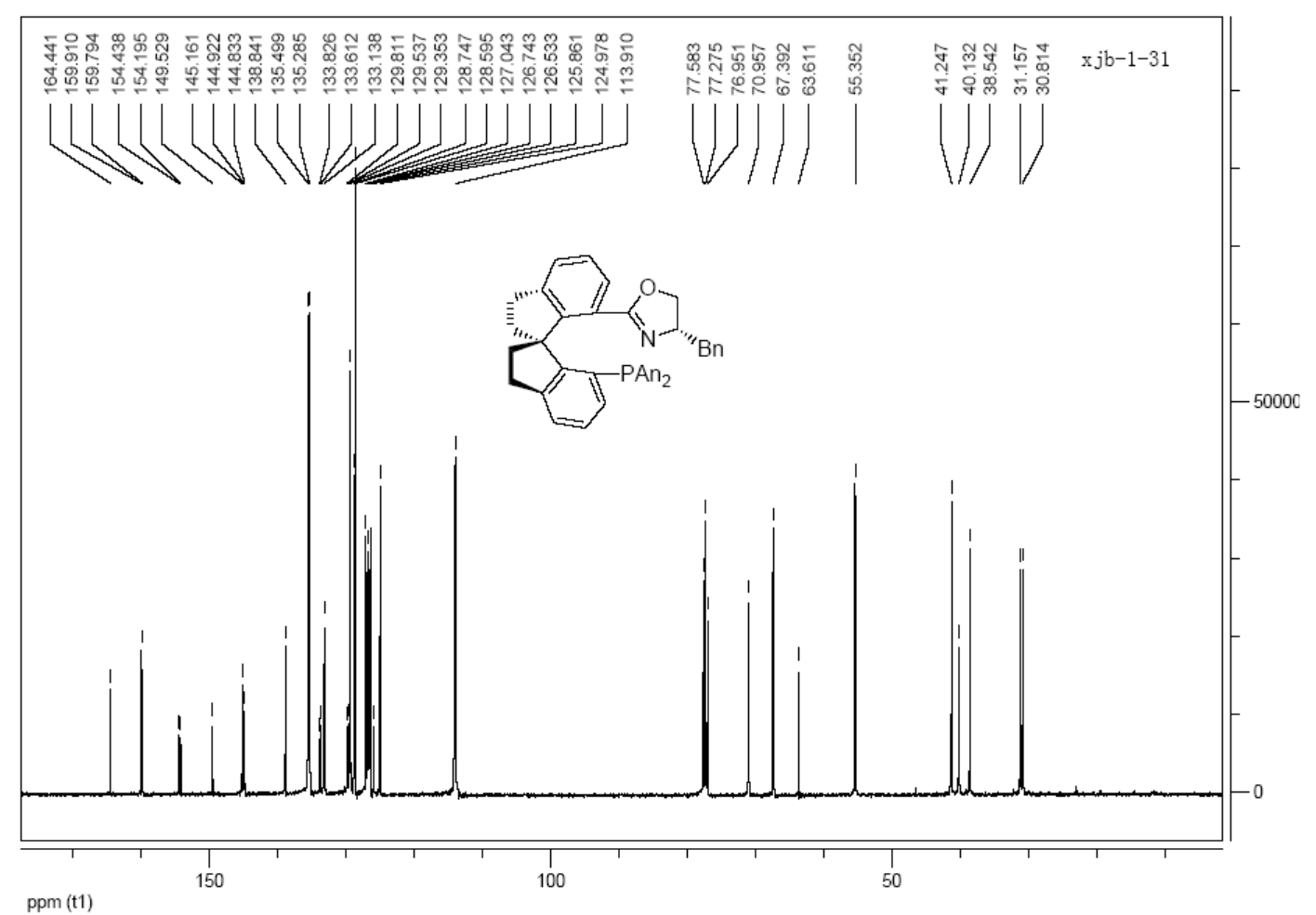

(S,S)-7-[4-(Benzyl)oxazol-2-yl]-7'-di(3,5-dimethylphenyl)phosphinyl-1,1'-spirobiinane ((S,S)-7e): Colorless oil and solidified with standing, 70\% yield. $[\alpha]^{24}-196.8$ (c 0.51 , $\mathrm{CH}_{2} \mathrm{Cl}_{2}$ ); ${ }^{1} \mathrm{H}$ NMR (300 MHz, $\left.\mathrm{CDCl}_{3}\right) \delta 7.60$ (d, $J=7.5 \mathrm{~Hz}, 1 \mathrm{H}, \mathrm{Ar}-\mathrm{H}$ ), 7.36-6.86 (m, 12H, Ar-H), 6.77 (d, $J=7.8 \mathrm{~Hz}, 2 \mathrm{H}, \mathrm{Ar}-\mathrm{H}), 6.60$ (d, $J=7.5 \mathrm{~Hz}, 2 \mathrm{H}, \mathrm{Ar}-\mathrm{H}) ; 4.14-4.04$ (m, 1H, $\mathrm{CH}_{2}$ ), 3.49 (dd, $J=8.4$ and $6.6 \mathrm{~Hz}, 1 \mathrm{H}, \mathrm{CH}_{2}$ ), 3.32 (t, $\left.J=8.8 \mathrm{~Hz}, 1 \mathrm{H}, \mathrm{CH}\right), 3.05-2.91\left(\mathrm{~m}, 3 \mathrm{H}, \mathrm{CH}_{2}\right.$ ), 2.82-2.63 (m, 3H, $\mathrm{CH}_{2}$ ), 2.20 (s, 6H, $\mathrm{CH}_{3}$ ), 2.18 (s, 6H, $\mathrm{CH}_{3}$ ), 2.10-1.86 (m, 4H, $\mathrm{CH}_{2}$ ); ${ }^{31} \mathrm{P}$ NMR (121 MHz, $\left.\mathrm{CDCl}_{3}\right) \delta-20.6$ (s); $\left.{ }^{13} \mathrm{C} \mathrm{NMR} \mathrm{(100} \mathrm{MHz,} \mathrm{CDCl}_{3}\right) \delta$ 164.1, 155.2, 155.0, 149.7, 145.3, 144.7, 138.9, 137.4, 137.2, 133.6, 131.9, 131.6, 130.0, 129.9, 129.3, 128.8, 127.0, 126.6, 126.4, 124.9, 70.8, 67.4, 63.7, 41.2, 40.4, 38.6, 31.2, 30.8, 21.5; MS (EI) m/z 619 (M, 43), 528 (100); HRMS (EI) calcd for $\mathrm{C}_{43} \mathrm{H}_{42} \mathrm{NOP}$ : 619.3004; Found 619.2978. 

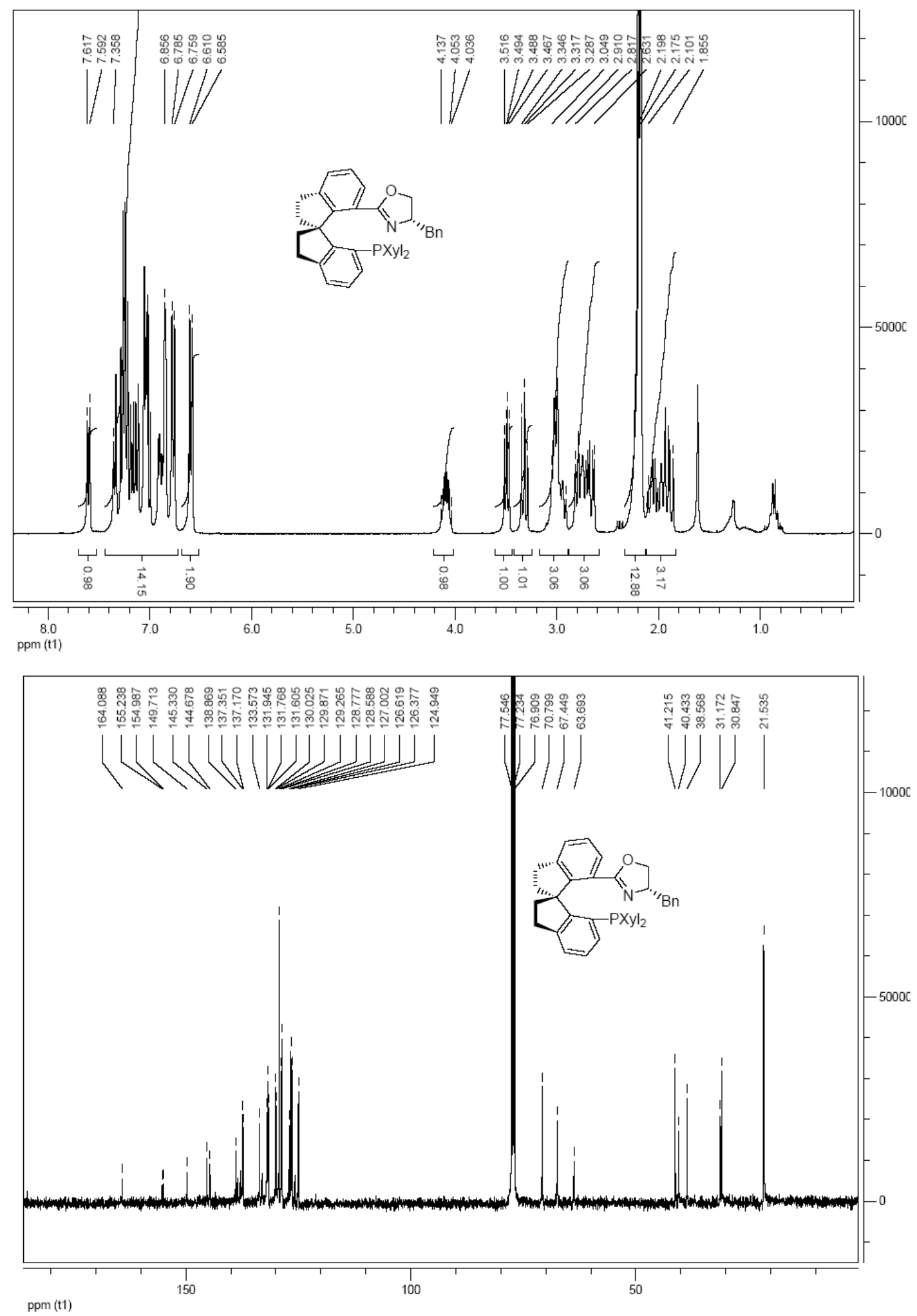

\section{Analytical Data of Iridium Complexes}

(S,S)-1a: An orange solid, 80\% yield. Mp 170-172 ${ }^{\circ} \mathrm{C}$; $[\alpha]^{15}{ }_{\mathrm{D}}+186.7$ (c $\left.0.58, \mathrm{CH}_{2} \mathrm{Cl}_{2}\right) ;{ }^{1} \mathrm{H}$ NMR (300 MHz, $\left.\mathrm{CDCl}_{3}\right) \delta 8.19$ (d, $\left.J=6.9 \mathrm{~Hz}, 1 \mathrm{H}\right), 7.72-7.20$ (m, $\left.26 \mathrm{H}\right), 6.64$ (s, 1H), 4.71 (s, 1H), 4.25-4.20 (m, 1H), 3.88 (s, 2H), 3.55-3.51 (m, 1H), 3.02-2.62 (m, 5H), 2.51-1.82 (m, 7H), 1.62-1.17 (m, 6H), 0.87 (dd, $J=21.6$ and $6.3 \mathrm{~Hz}, 6 \mathrm{H}), 0.49-0.37$ (m, 1H); ${ }^{31} \mathrm{P}$ NMR 
$\left(121 \mathrm{MHz}, \mathrm{CDCl}_{3}\right) \delta 15.3$ (s); ${ }^{13} \mathrm{C}$ NMR (100 MHz, $\left.\mathrm{CDCl}_{3}\right) \delta 173.3,162.7,162.2,161.7$, 161.2, 148.9, 148.8, 148.1, 148.0, 146.6, 144.4, 135.0, 133.5, 133.4, 133.2, 132.7, 132.4, $130.9,130.7,129.7,129.6,129.3,129.0,128.9,128.7,128.6,128.4,128.3,127.8,127.4$, 127.3, 127.1, 126.1, 124.6, 123.4, 121.1, 120.7, 117.1, 75.7, 72.2, 71.7, 70.8, 68.5, 63.0, 41.4, 34.4, 32.2, 31.3, 31.0, 30.4, 30.0, 29.5, 19.2, 14.5, 1.3; MS (ESI) m/z $816\left(\mathrm{M}^{+}, 40\right), 706$ (100); Anal. calcd for $\mathrm{C}_{75} \mathrm{H}_{58} \mathrm{BF}_{24} \mathrm{IrNOP}$ : C 53.64, $\mathrm{H}$ 3.48, N 0.83; Found: C 53.64, H 3.34, N 0.80.

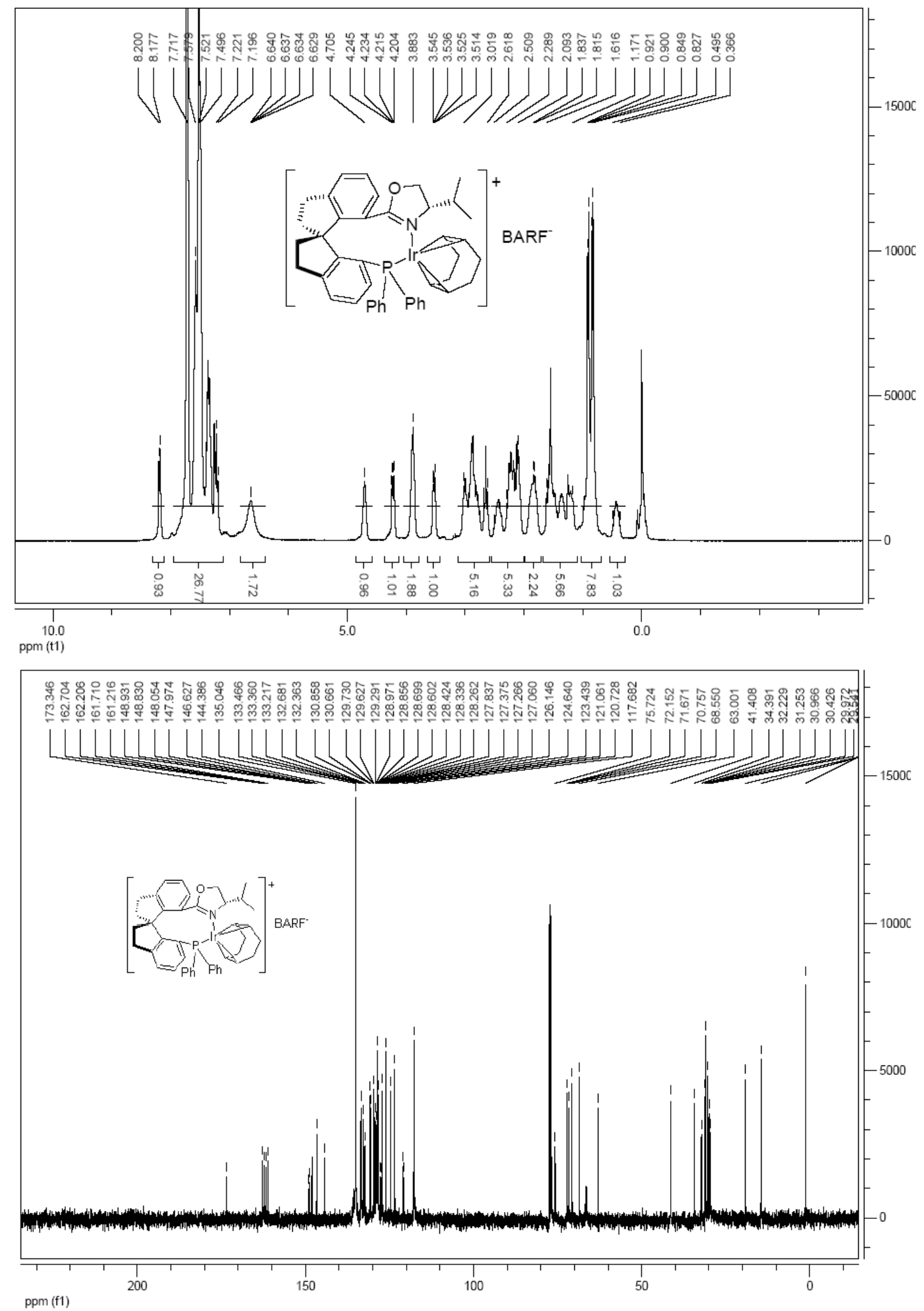




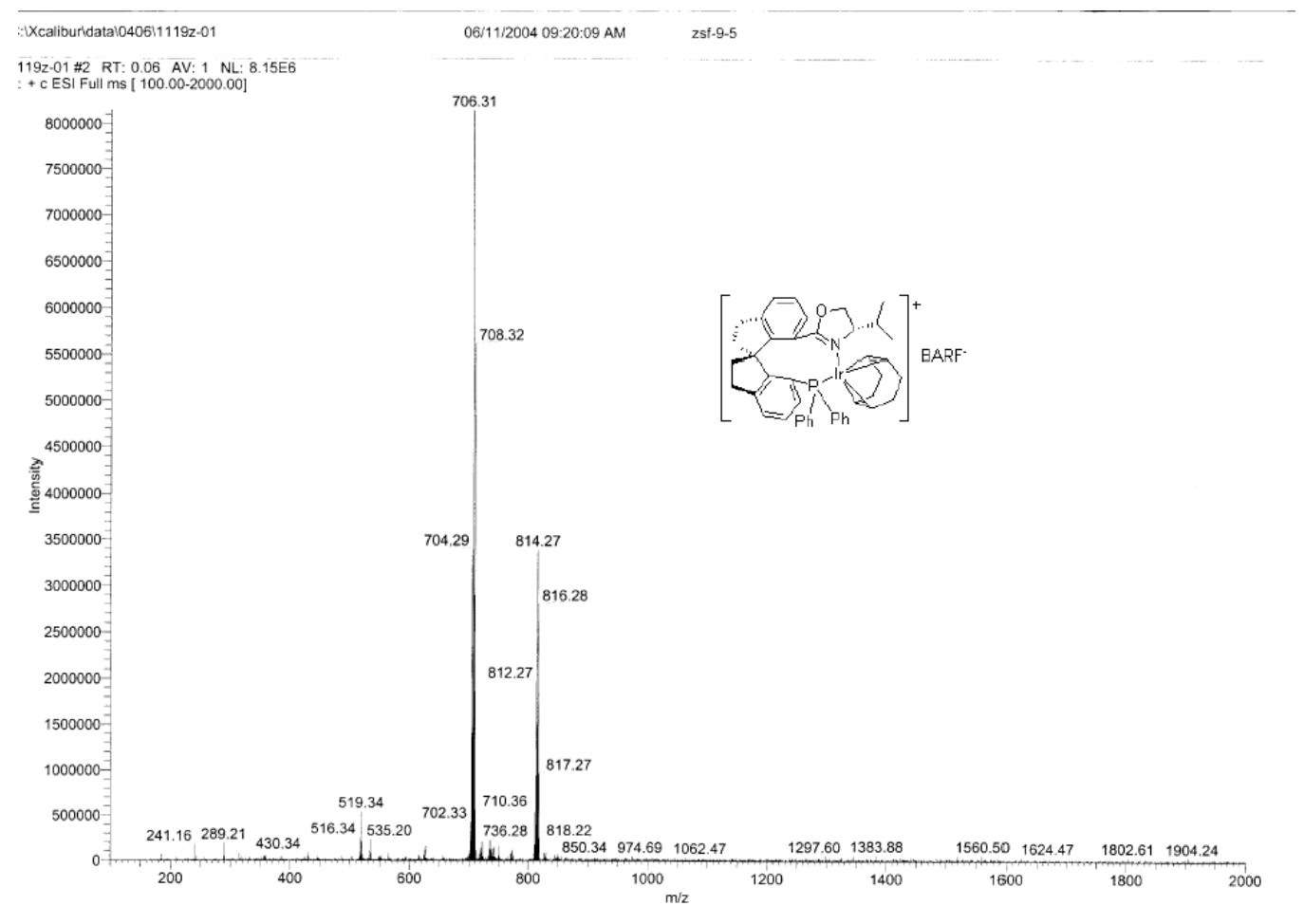

(S,R)-1a: An orange solid, 80\% yield. Mp 177-179 ${ }^{\circ} \mathrm{C}$; $[\alpha]^{15}{ }_{\mathrm{D}}-52.2\left(\mathrm{c} 0.45, \mathrm{CH}_{2} \mathrm{Cl}_{2}\right) ;{ }^{1} \mathrm{H}$ NMR (300 MHz, $\left.\mathrm{CDCl}_{3}\right) \delta 7.72$ (s, 8H), 7.53-7.23 (m, $\left.18 \mathrm{H}\right), 7.08-7.02$ (m, 2H), 4.70-4.63 (m, 1H), 4.56-4.44 (m, 2H), 3.94-3.84 (m, 1H), 3.62-3.55 (m, 1H), 3.40-3.55 (m, 1H), 2.99-2.82 (m, 2H), 2.61-2.49 (m, 1H); 2.37-2.04 (m, 5H), 1.98-1.81 (m, 2H), 1.62 (d, J = 6.6 Hz, 3H), 0.46 (d, $J=6.6 \mathrm{~Hz}, 3 \mathrm{H}) ;{ }^{31} \mathrm{P}$ NMR (121 MHz, $\left.\mathrm{CDCl}_{3}\right) \delta 14.2(\mathrm{~s}) ;{ }^{13} \mathrm{C}$ NMR (100 $\left.\mathrm{MHz}, \mathrm{CDCl}_{3}\right) \delta 173.7,162.7,162.2,161.7,161.2$, 149.7, 149.6, 147.4, 147.2, 144.0, 137.3, 137.2, 135.0, 134.6, 134.5, 132.6, 132.1, 131.1, 129.7, 129.5, 129.3, 129.1, 129.0, 128.7, 128.6, 128.4, 128.3, 127.5, 126.9, 126.5, 126.1, 123.4, 120.7, 117.6, 85.0, 84.8, 83.6, 83.5, 73.4, 72.2, 71.7, 65,6, 63.9, 40.0, 36.1, 33.0, 32.3, 30.6, 30.0, 29.6, 28.6, 24.7, 17.2; MS (ESI) m/z $816\left(\mathrm{M}^{+}, 20\right), 706$ (100); Anal. calcd for $\mathrm{C}_{75} \mathrm{H}_{58} \mathrm{BF}_{24} \mathrm{IrNOP}$ : C 53.64, H 3.48, N 0.83; Found: C 53.64, H 3.34, N 0.80 . 

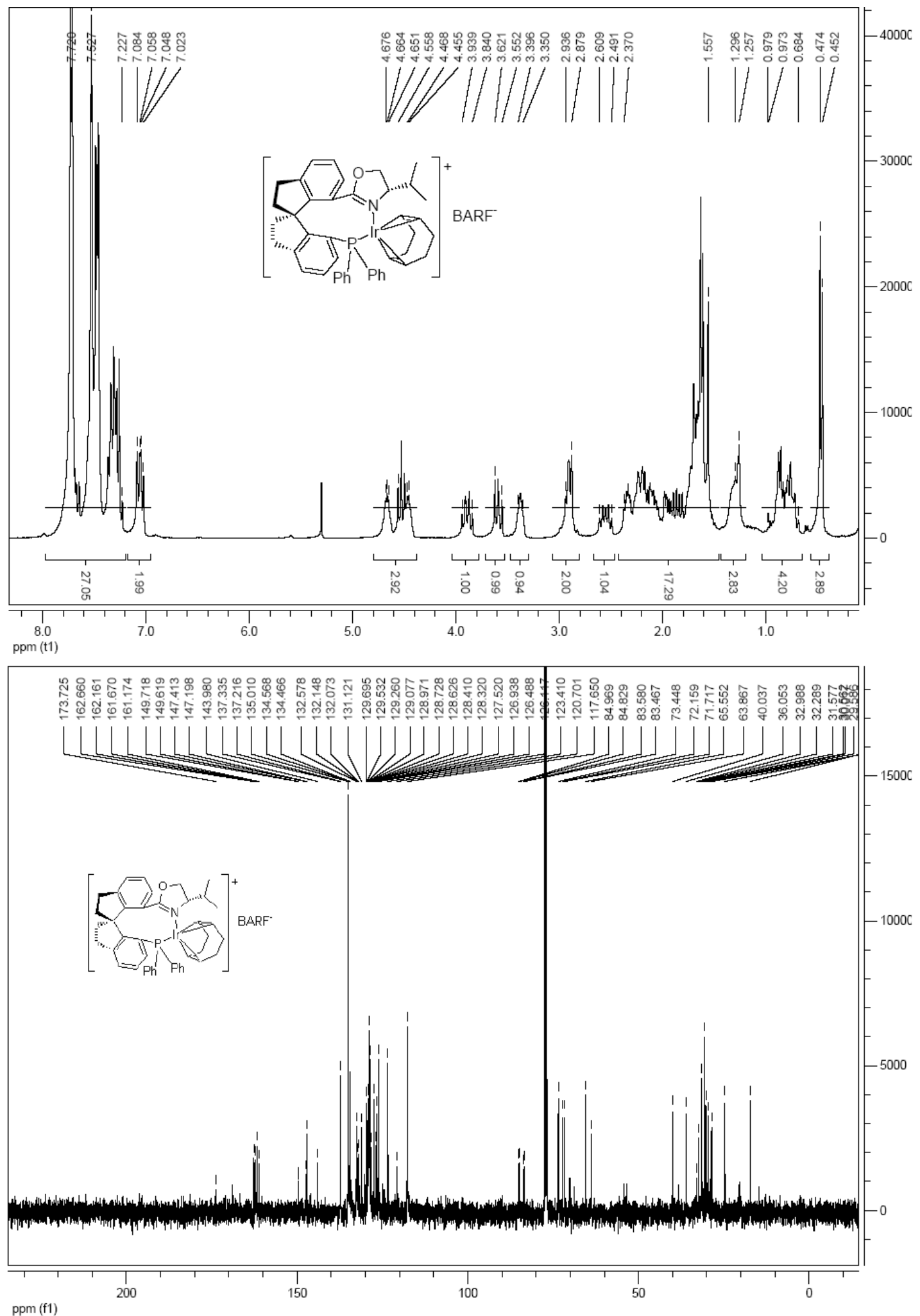


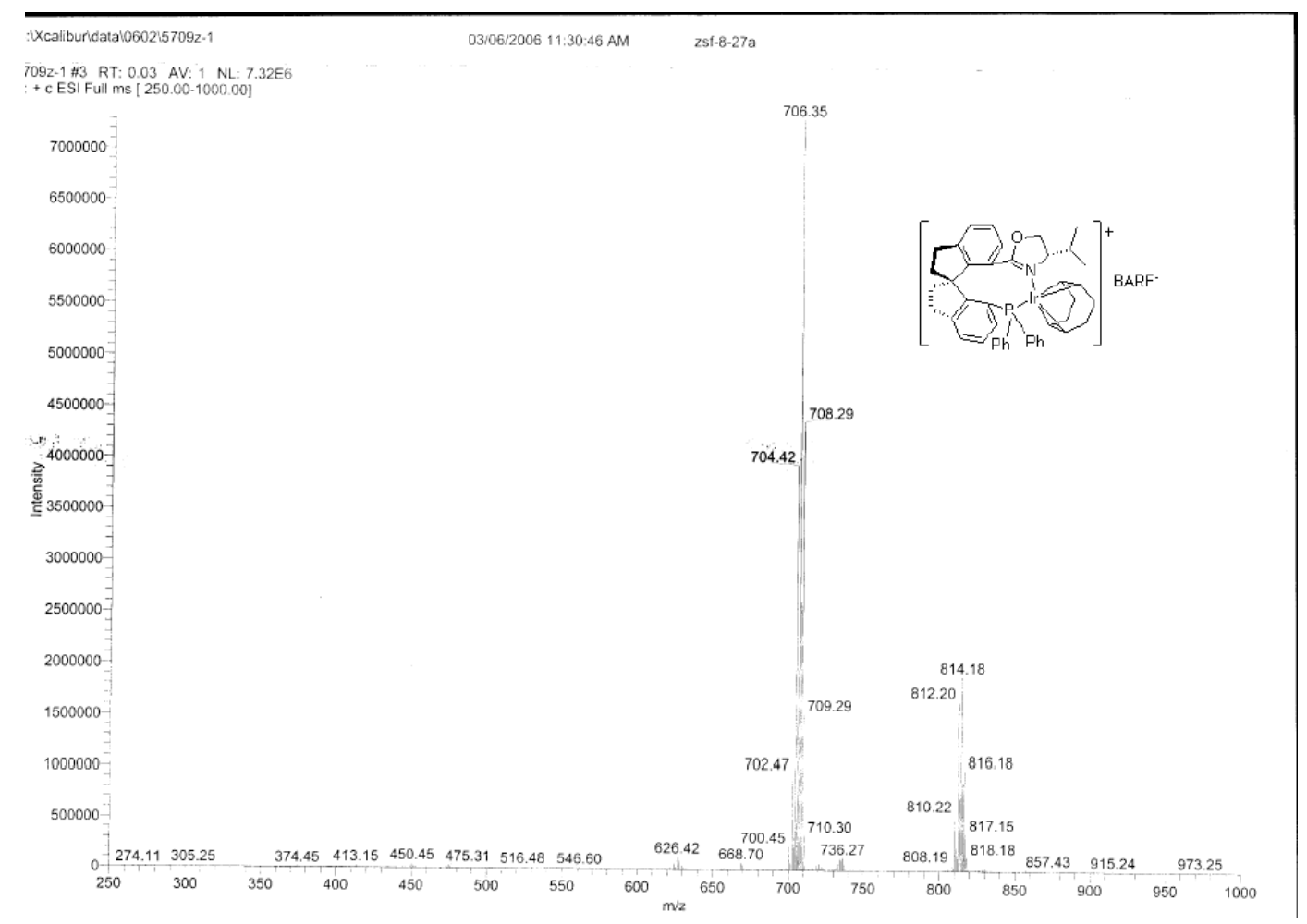

(S,S)-1b: An orange solid, 85\% yield. Mp 166-167 ${ }^{\circ} \mathrm{C}$; $[\alpha]^{15}{ }_{\mathrm{D}}+191.7\left(\mathrm{c} 0.54, \mathrm{CH}_{2} \mathrm{Cl}_{2}\right) ;{ }^{1} \mathrm{H}$ NMR (300 MHz, $\left.\mathrm{CDCl}_{3}\right) \delta 8.06$ (d, $\left.J=7.2 \mathrm{~Hz}, 1 \mathrm{H},\right), 7.77-7.26(\mathrm{~m}, 30 \mathrm{H}), 6.82-6.70$ (m, 2H), 4.46-4.31 (m, 2H), 3.90-3.70 (m, 2H), 3.65-3.50 (m, 1H), 3.40 (t, $J=9.3 \mathrm{~Hz}, 1 \mathrm{H}), 2.96-2.73$ (m, 4H), 2.34-2.01 (m, 5H), 1.71-1.54 (m, 1H), 1.37-1.15 (m, 5H), 0.97-0.76 (m, 2H); ${ }^{31} \mathrm{P}$ NMR (121 MHz, $\left.\mathrm{CDCl}_{3}\right) \delta 14.5(\mathrm{~s}) ;{ }^{13} \mathrm{C} \mathrm{NMR}\left(100 \mathrm{MHz}, \mathrm{CDCl}_{3}\right) \delta 171.8,162.7,162.2$, $161.7,161.2$, 148.6, 148.5, 148.4, 148.3, 146.4, 144.4, 138.0, 135.9, 135.0, 133.8, 133.7, $132.9,132.8,132.4,132.3,130.9,130.7,130.3,130.1,129.7,129.6,129.3,129.0,128.8$, 128.7, 128.6, 128.5, 128.4, 128.0, 127.6, 127.3, 126.9, 126.1, 125.9, 123.4, 121.3, 120.7, 117.7, 79.7, 78.6, 72.5, 72.3, 70.5, 69.8, 69.1, 63.3, 41.6, 34.3, 31.7, 30.9, 30.4, 29.8, 29.1; MS (ESI) m/z 850 (M+68), 740 (100); Anal. calcd for $\mathrm{C}_{78} \mathrm{H}_{56} \mathrm{BF}_{24} \mathrm{IrNOP}$ : C 54.68, H 3.29, $\mathrm{N}$ 0.82; Found: C 54.70, H 3.10, N 0.85. 

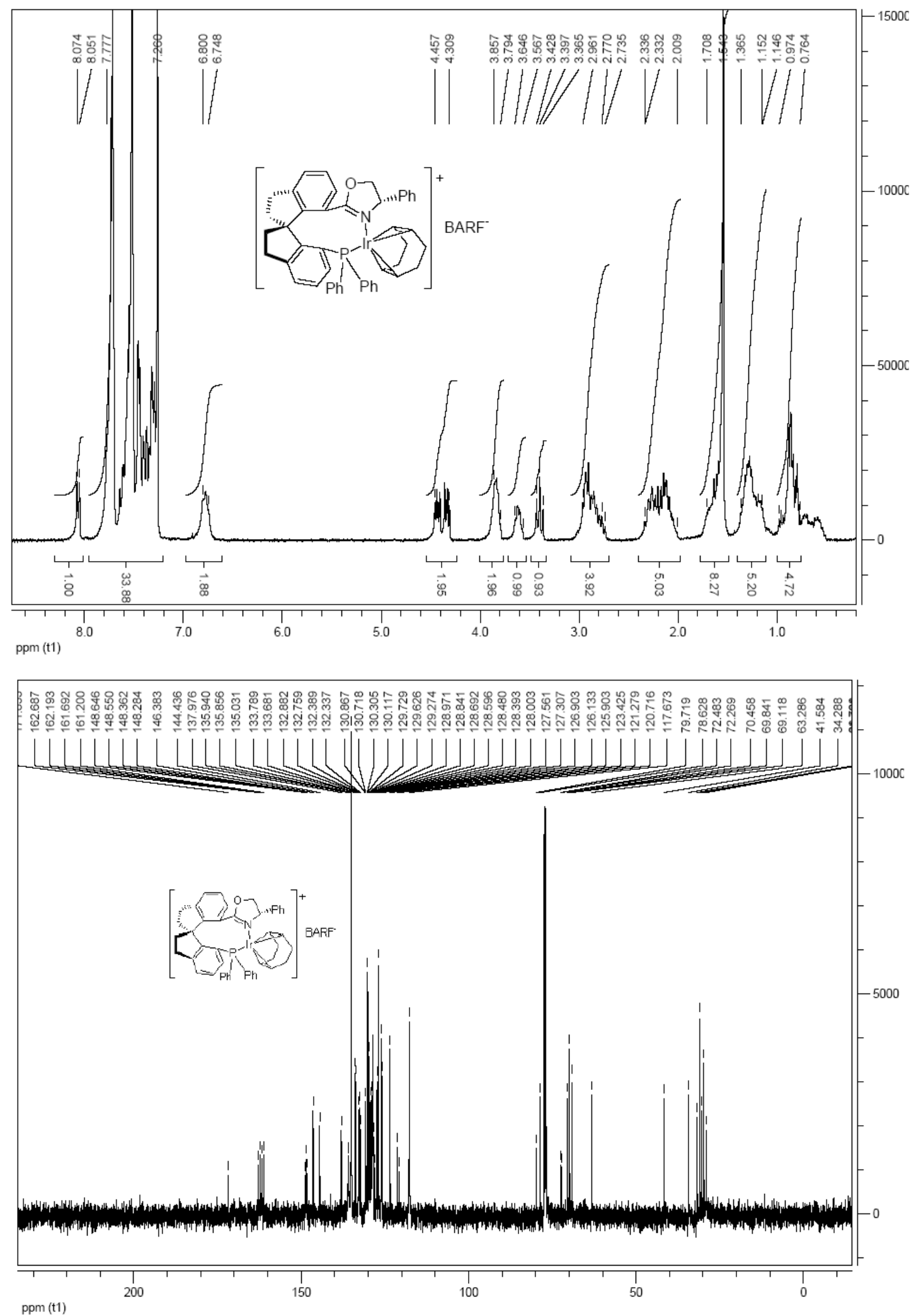


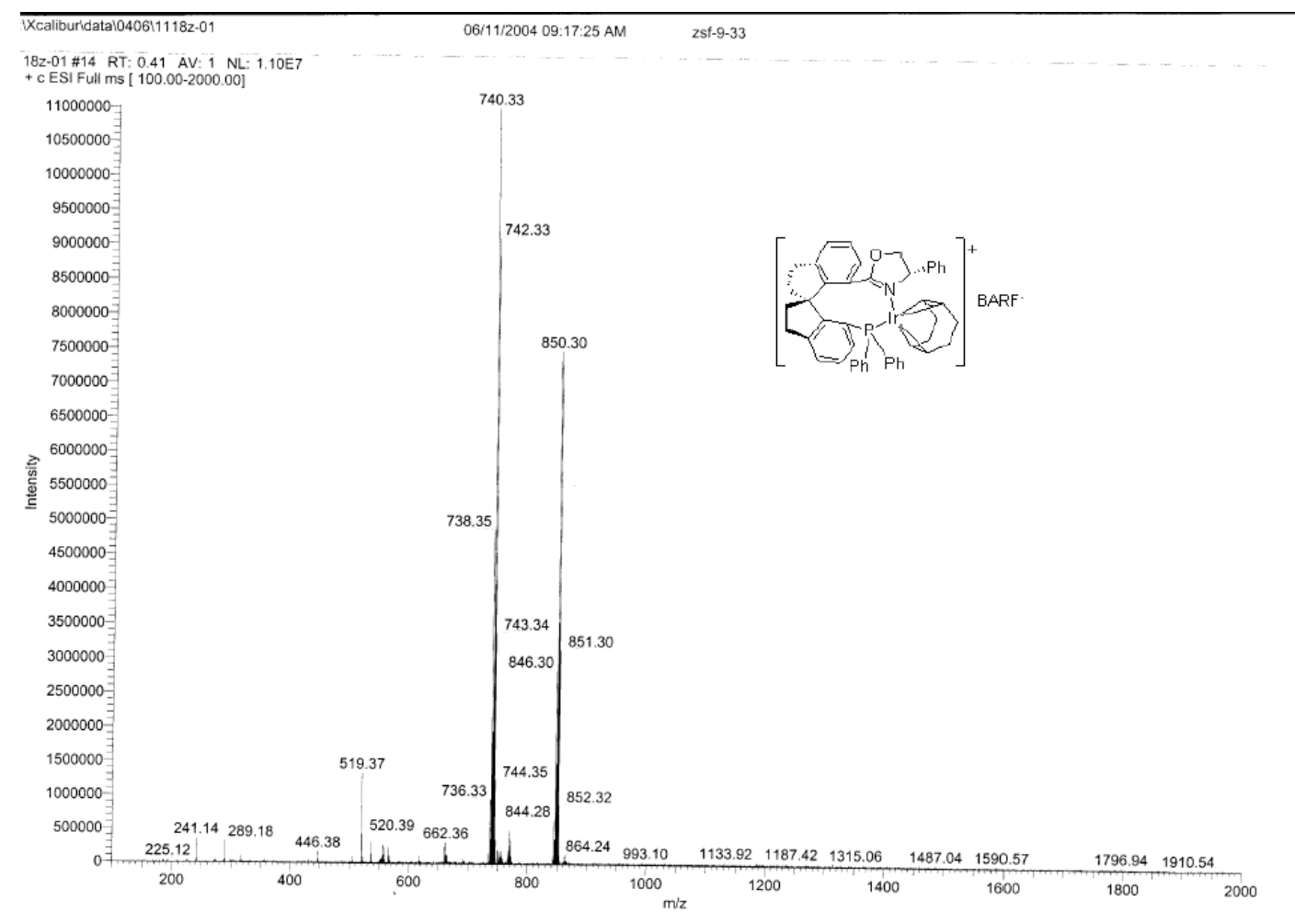

(S,S)-1c: An orange-red solid, 90\% yield. Mp 178-179 ${ }^{\circ} \mathrm{C}$; $[\alpha]^{15}{ }_{\mathrm{D}}+168.6\left(\mathrm{c} 0.5, \mathrm{CH}_{2} \mathrm{Cl}_{2}\right) ;{ }^{1} \mathrm{H}$ NMR (300 MHz, $\left.\mathrm{CDCl}_{3}\right) \delta 8.11$ (d, $\left.J=6.9 \mathrm{~Hz}, 1 \mathrm{H}\right), 7.73-7.51(\mathrm{~m}, 16 \mathrm{H}), 7.39-7.07$ (m, 8H), 6.71 (s, 2H), 4.91-4.86 (m, 1H), 4.20-4.10 (m, 2H), 3.91-3.85 (m, 1H), 3.79-3.69 (m, 1H), 3.17-3.64 (m, 6H), 2.63-2.40 (m, 1H), 2.32-2.04 (m, 4H), 1.98-1.86 (m, 1H), 1.60-1.26 (m, $4 \mathrm{H}), 0.90-0.76$ (m, 4H). ${ }^{31} \mathrm{P}$ NMR (121 MHz, $\left.\mathrm{CDCl}_{3}\right) \delta 14.9(\mathrm{~s}) ;{ }^{13} \mathrm{C}$ NMR $\left(100 \mathrm{MHz}, \mathrm{CDCl}_{3}\right)$ $\delta$ 173.1, 162.7, 162.2, 161.7, 161.2, 148.7, 148.2, 146.6, 144.5, 135.8, 135.7, 135.1, 134.4, 133.6, 133.5, 132.9, 132.8, 132.4, 132.3, 130.8, 130.7, 129.8, 129.6, 128.9, 128.7, 128.5, 128.4, 128.1, 127.6, 127.3, 127.2, 126.8, 126.2, 125.3, 123.5, 121.1, 120.7, 117.7, 76.6, 75.2, 71.6, 69.2, 67.1, 63.2, 41.5, 41.0, 34.5, 32.1, 30.9, 30.4, 30.2, 29.4; MS (ESI) m/z $864\left(\mathrm{M}^{+}\right.$, 68), 754 (100); Anal. calcd for $\mathrm{C}_{79} \mathrm{H}_{58} \mathrm{BF}_{24} \mathrm{IrNOP}$ : C 54.93, H 3.38, N 0.81; Found: C 54.95, H 3.19, N 0.87 . 

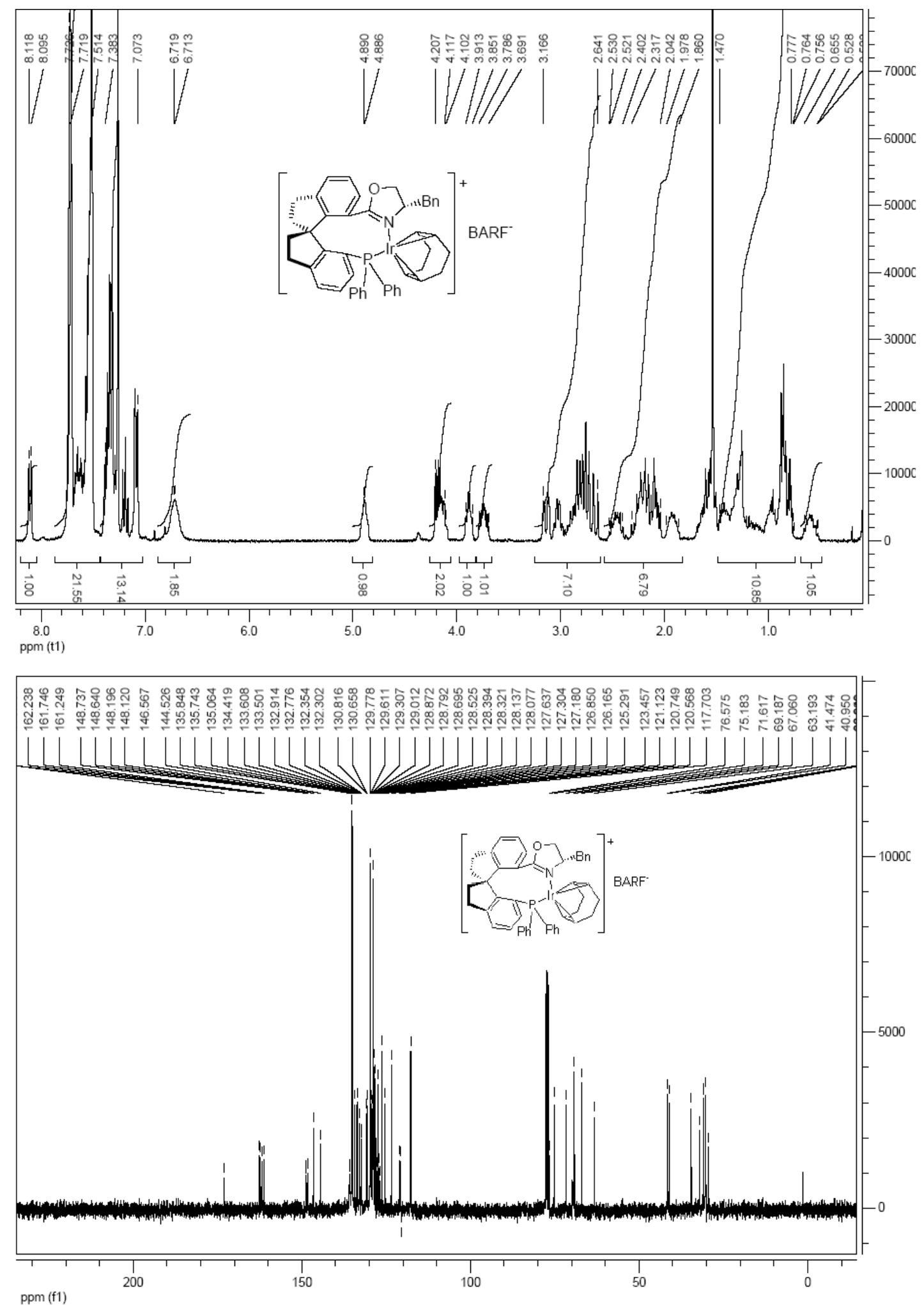


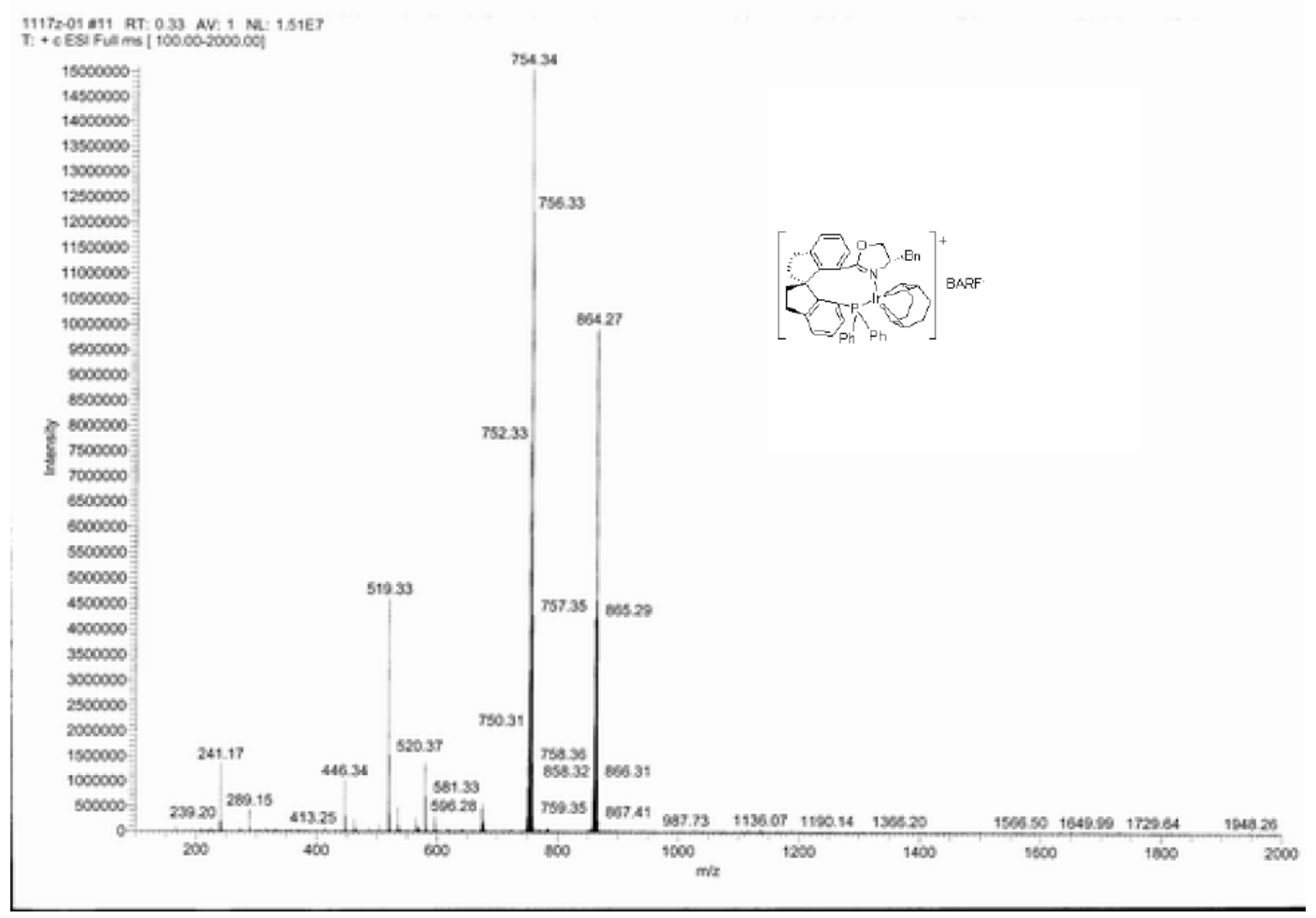

(S,S)-1d: An orange solid, 95\% yield. Mp 83-84 ${ }^{\circ} \mathrm{C}$; $[\alpha]^{15}{ }_{\mathrm{D}}+136.6\left(\mathrm{c} 0.5, \mathrm{CH}_{2} \mathrm{Cl}_{2}\right) ;{ }^{1} \mathrm{H}$ NMR $\left(300 \mathrm{MHz}, \mathrm{CDCl}_{3}\right) \delta 8.05-8.66(\mathrm{~m}, 31 \mathrm{H}), 4.87(\mathrm{~s}, 1 \mathrm{H}) ; 4.17-4.12(\mathrm{~m}, 1 \mathrm{H}), 3.84$ (d, $J=11.7$ $\mathrm{Hz}, 6 \mathrm{H}), 3.69-3.65(\mathrm{~m}, 1 \mathrm{H}), 3.20-2.63(\mathrm{~m}, 6 \mathrm{H}), 2.49-2.37(\mathrm{~m}, 1 \mathrm{H}), 2.33-2.02(\mathrm{~m}, 3 \mathrm{H})$, 1.92-1.59 (m, 3H), 1.40-1.16 (m, 3H), 1.14-0.79 (m, 4H), 0.31-0.20 (m, 1H); ${ }^{31}$ P NMR (121 $\left.\mathrm{MHz}, \mathrm{CDCl}_{3}\right) \delta 12.1(\mathrm{~s}) ;{ }^{13} \mathrm{C} \mathrm{NMR}\left(75 \mathrm{MHz}, \mathrm{CDCl}_{3}\right) \delta 172.5,172.4,162.8,162.7,162.0$, 161.3, 160.7, 148.3, 148.2, 147.9, 147.8, 146.1, 144.7, 137.3, 137.1, 135.1, 134.8, 134.2, $131.9,130.2$, 130.0, 129.5, 129.4, 129.3, 128.6, 128.1, 127.9, 127.7, 127.0, 126.4, 125.3, 123.5, 122.8, 121.0, 119.1, 118.5, 117.5, 117.4, 115.1, 114.9, 114.2, 114.0, 74.8, 70.9, 70.6, 70.4, 69.0, 66.9, 63.0, 55.5, 55.4, 41.4, 40.7, 34.5, 31.7, 30.9, 30.6, 30.5, 29.9, 29.2; MS (ESI) m/z $924\left(\mathrm{M}^{+}, 40\right), 814$ (100); Anal. calcd for $\mathrm{C}_{81} \mathrm{H}_{62} \mathrm{BF}_{24} \mathrm{IrNO}_{3} \mathrm{P}$ : C 54.43, H 3.50, N 0.78; Found: C 54.43, H 3.46, N 1.00 . 


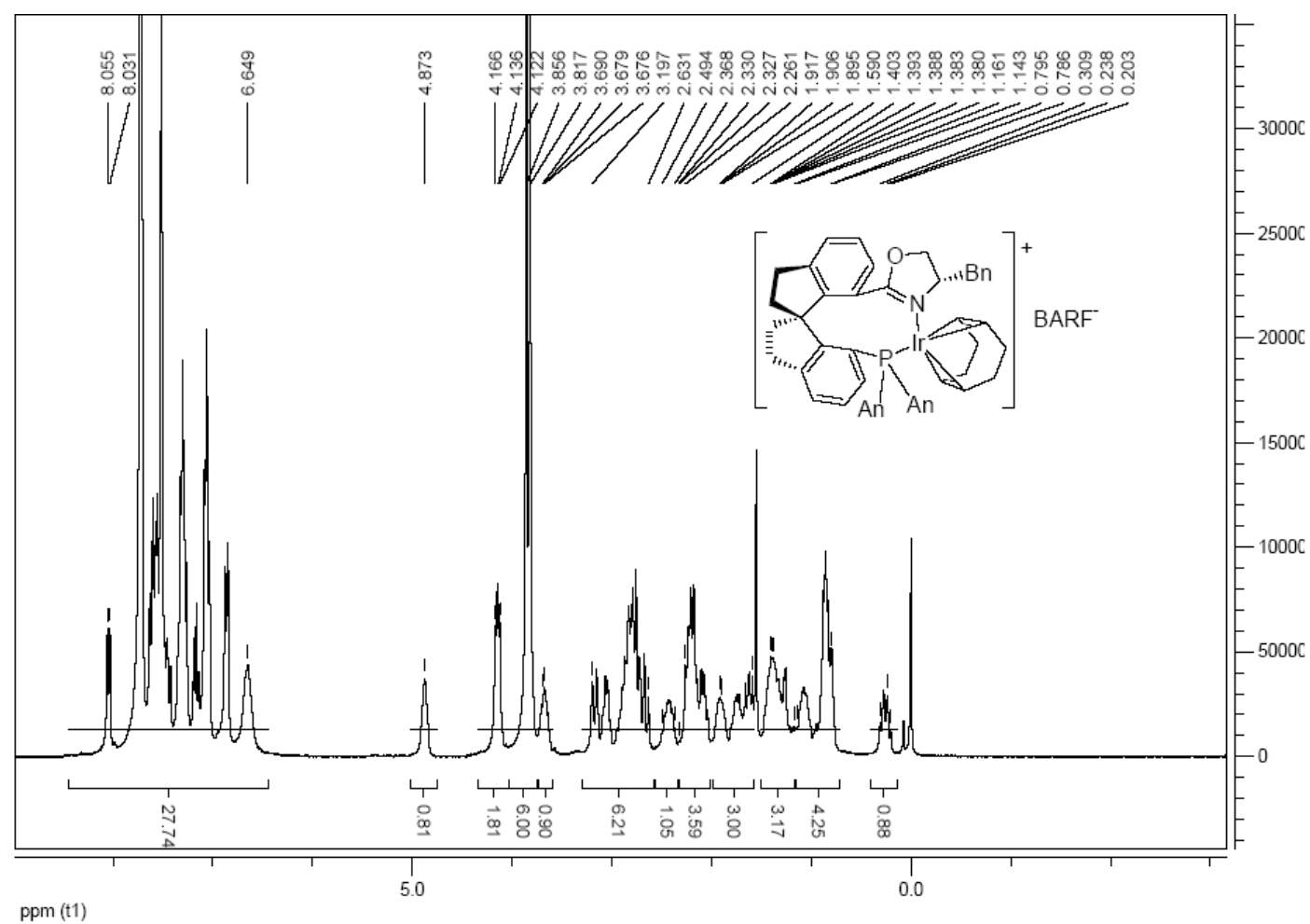

ppm (t1)

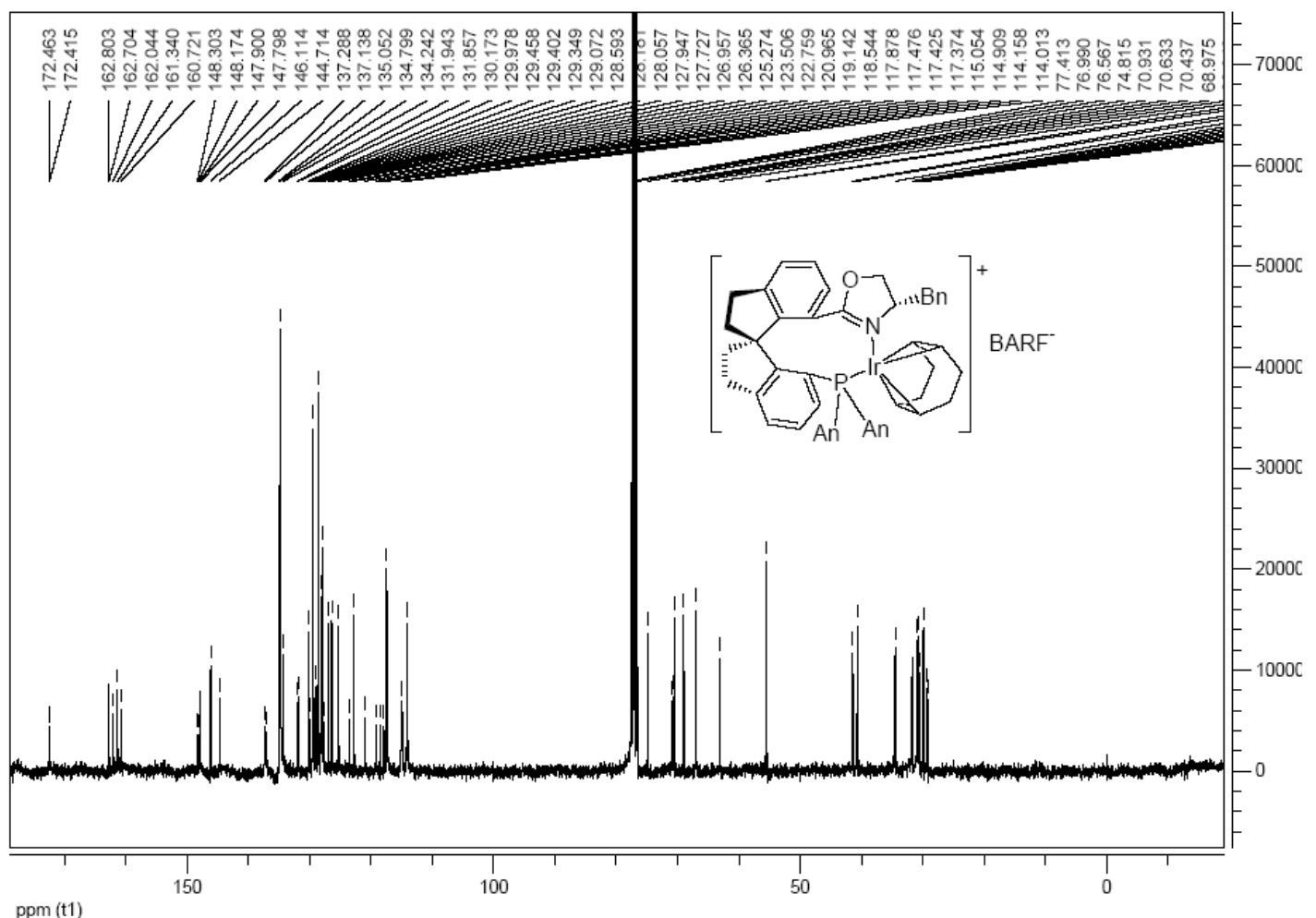




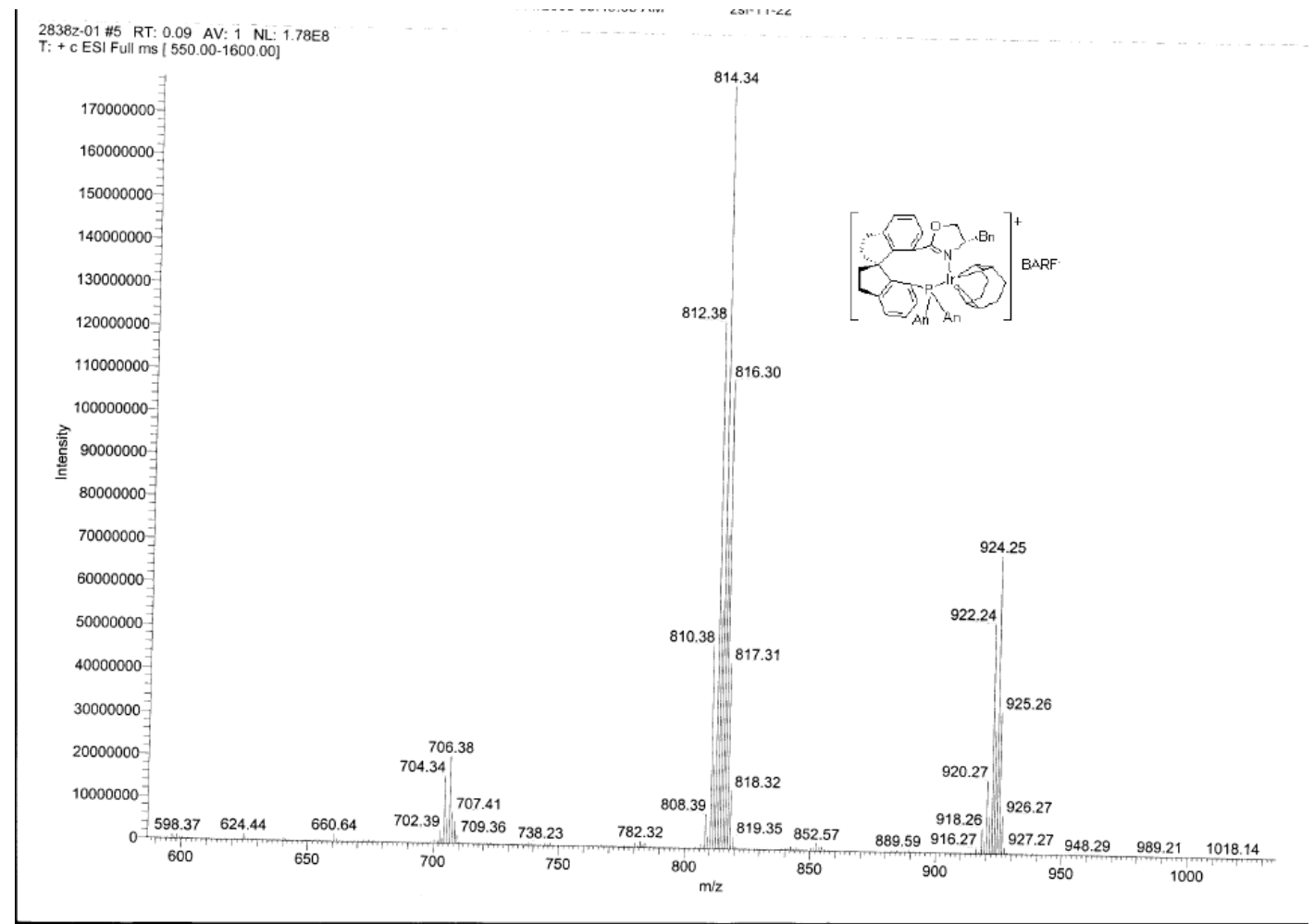

(S,S)-1e: An orange solid, 92\% yield. Mp 177-179 ${ }^{\circ} \mathrm{C}$; $[\alpha]^{15}{ }_{\mathrm{D}}+174.6\left(\mathrm{c} 0.5, \mathrm{CH}_{2} \mathrm{Cl}_{2}\right)$; ${ }^{1} \mathrm{H}$ NMR (300 MHz, $\left.\mathrm{CDCl}_{3}\right) \delta 8.07(\mathrm{~d}, J=7.2 \mathrm{~Hz}, 1 \mathrm{H}), 7.72(\mathrm{~s}, 8 \mathrm{H}), 7.59-7.51(\mathrm{~m}, 7 \mathrm{H})$, 7.34-7.06 (m, 13H), 4.80 (s, 1H), 4.18-4.12 (m, 2H), 3.80-3.67 (m, 2H), 3.20-3.16 (m, 1H), 3.01-2.65 (m, 6H), 2.52-2.03 (m, 15H), 1.63-1.52 (m, 1H), 1.39-1.25 (m, 4H), 1.04-0.76 (m, 4H), 0.67-0.57 (m, 1H); ${ }^{31} \mathrm{P}$ NMR (121 MHz, $\left.\mathrm{CDCl}_{3}\right) \delta 14.8(\mathrm{~s}) ;{ }^{13} \mathrm{C}$ NMR (100 MHz, CDCl $\left.{ }_{3}\right)$ $\delta$ 172.8, 162.8, 162.3, 161.8, 161.3, 148.5, 148.4, 148.0, 146.5, 144.8, 139.5, 138.4, 138.3, $135.1,134.7,134.3,132.5,132.3,131.8,131.6,131.5,130.0,129.6,129.4,129.1,128.9$, 128.8, 128.4, 128.1, 127.6, 127.3, 127.1, 126.8, 126.2, 125.4, 123.5, 121.2, 120.8, 117.7, 76.0, 75.2, 71.1, 70.3, 70.2, 70.1, 69.7, 67.4, 63.3, 41.5, 41.1, 34.2, 31.7, 30.8, 30.6, 30.0, 29.8, 21.8, 21.4; MS (ESI) m/z $920\left(\mathrm{M}^{+}, 10\right), 810$ (100); Anal. calcd for $\mathrm{C}_{83} \mathrm{H}_{66} \mathrm{BF}_{24} \mathrm{IrNOP}$ : C 55.90, H 3.73, N 0.79; Found: C 56.14, H 3.56, N 0.98. 


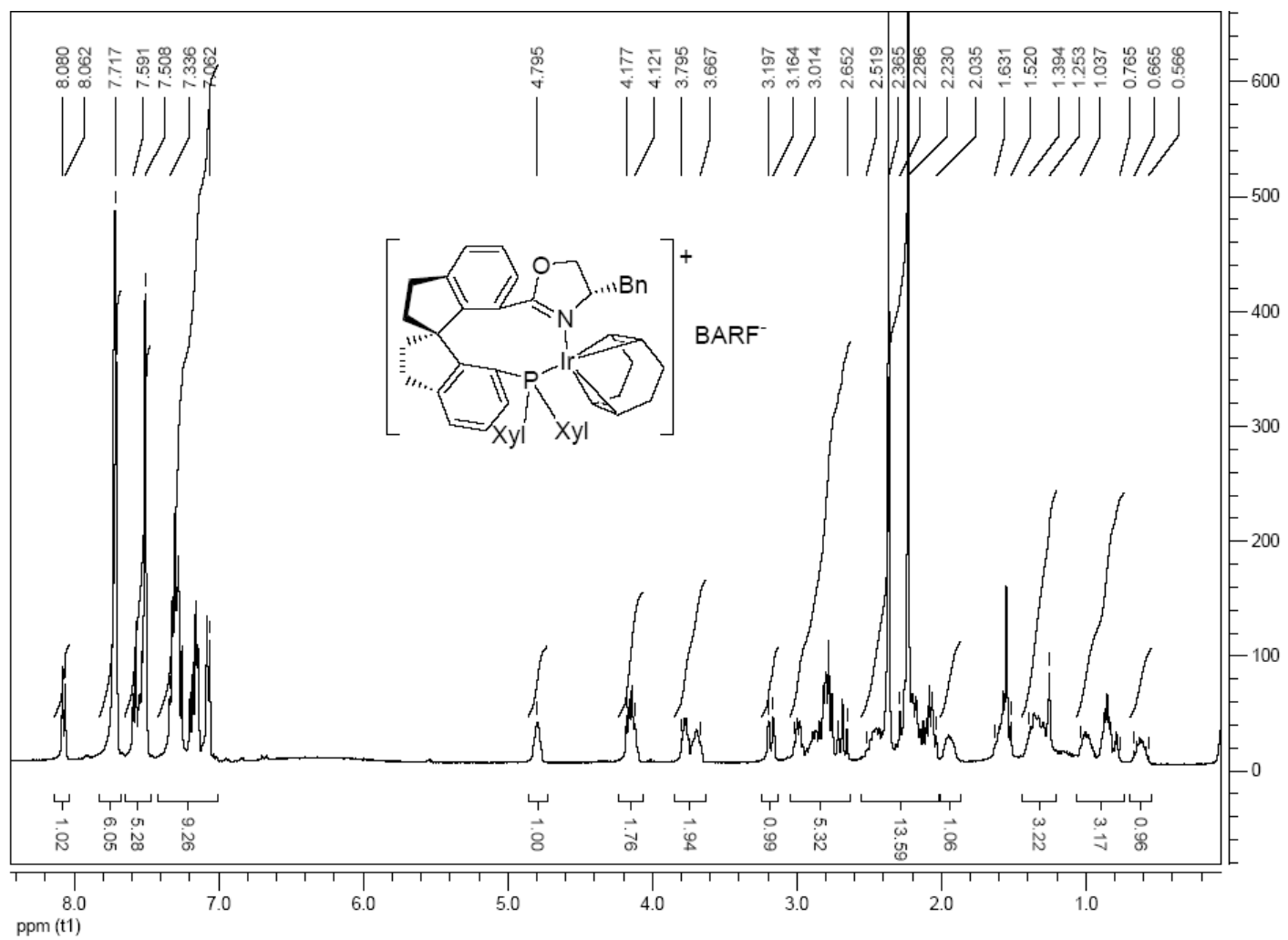

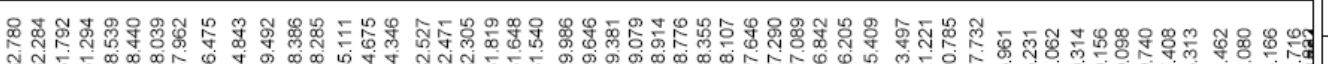

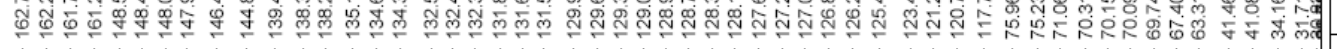

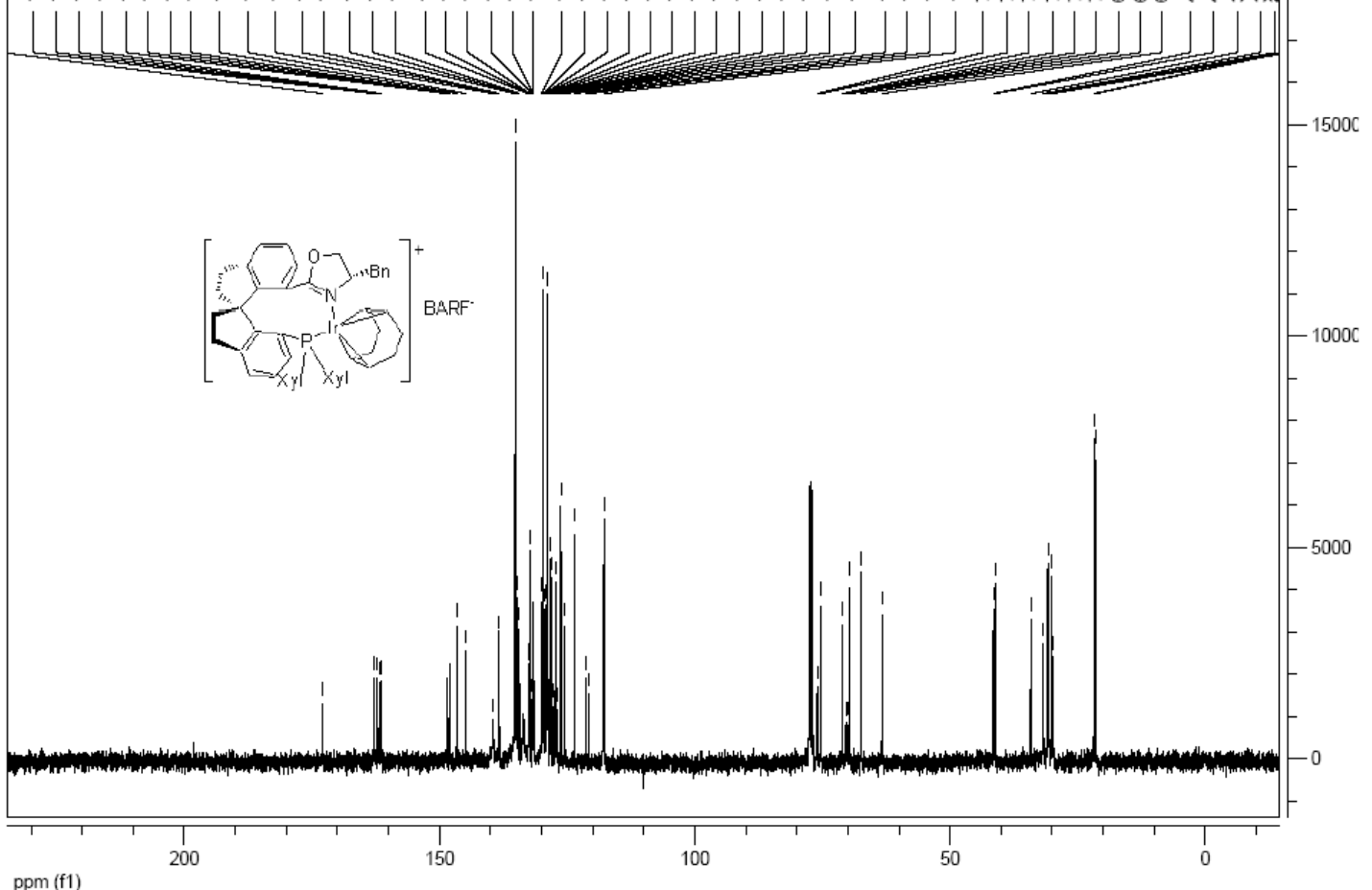




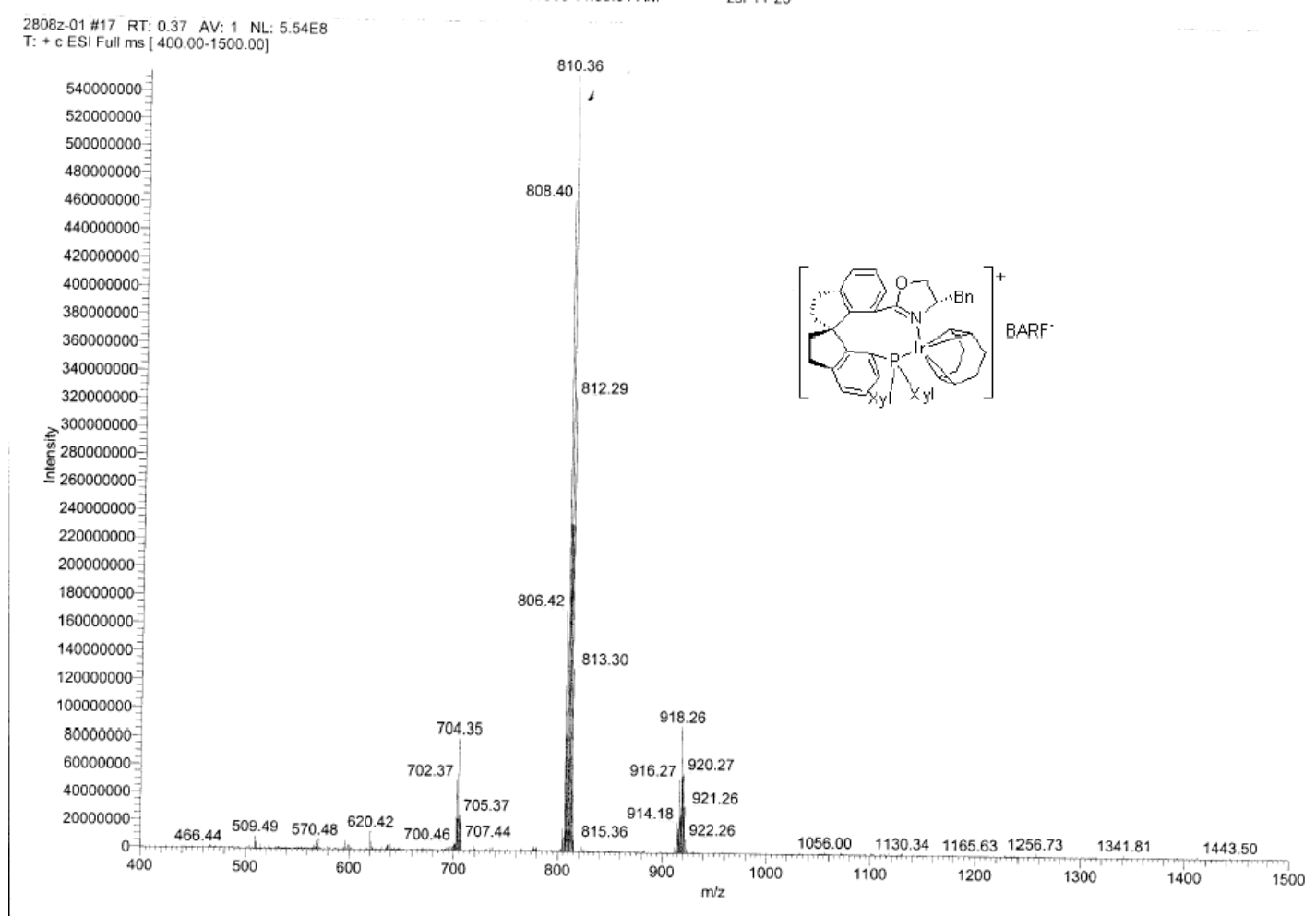

\section{X-Ray Analyses of Structures of Complexes $(S, S)-1 a$ and $(S, R)-1 a$}

The complexes were dissolved in ethanol and water was added carefully. The fine orange-red crystals grow slowly on the interface, which are suitable for the X-ray diffraction analyses.

Table 1 Crystal data and structure refinement for $(S, S)-\mathbf{1 a}$.

Empirical formula

Formula weight

Temperature

Wavelength

Crystal system, space group

Unit cell dimensions

$$
\mathrm{C}_{75} \mathrm{H}_{58} \mathrm{~B} \mathrm{~F}_{24} \text { Ir N O P }
$$

1679.20

293(2) K

$0.71073 \mathrm{~A}$
Orthorhombic, P2(1)2(1)2(1)

$$
\begin{aligned}
& a=13.888(4) A \\
& b=20.666(6) A \\
& c=25.416(8) A \\
& \text { alpha }=90 \text { deg. }
\end{aligned}
$$




$$
\begin{gathered}
\text { beta }=90 \mathrm{deg} . \\
\text { gamma }=90 \mathrm{deg} .
\end{gathered}
$$

Volume

Z, Calculated density

Absorption coefficient

F (000)

Crystal size

Theta range for data collection

Limiting indices

Reflections collected / unique

Completeness to theta $=28.00$

Absorption correction

Max. and min. transmission

Refinement method

Data / restraints / parameters

Goodness-of-fit on $\mathrm{F}^{\wedge} 2$

Final R indices [I>2sigma(I)]

$\mathrm{R}$ indices (all data)

Absolute structure parameter

Largest diff. peak and hole
7295(4) $A \wedge 3$

3, $1.555 \mathrm{Mg} / \mathrm{m}^{\wedge} 3$

$1.991 \mathrm{~mm}^{\wedge}-1$

2508

$0.22 \times 0.18 \times 0.16 \mathrm{~mm}$

1.67 to $28.00 \mathrm{deg}$.

$-17<=\mathrm{h}<=14$

$-15<=\mathrm{k}<=16$

$-48<=\mathrm{l}<=45$

$34642 / 16043[\mathrm{R}(\mathrm{int})=0.0370]$

$99.8 \%$

Semi-empirical from equivalents

1.000000 and 0.917897

Full-matrix least-squares on $\mathrm{F}^{\wedge} 2$

16043 / 457 / 1163

1.101

$\mathrm{R} 1=0.0529, \mathrm{wR} 2=0.1064$

$\mathrm{R} 1=0.0696, \mathrm{wR} 2=0.1119$

$0.003(5)$

0.673 and -1.933 e. $A^{\wedge}-3$ 
Table 2. Crystal data and structure refinement for $(S, R)-\mathbf{1 a}$.

Empirical formula

Formula weight

Temperature

Wavelength

Crystal system, space group

Unit cell dimensions

Volume

Z, Calculated density

Absorption coefficient

F (000)

Crystal size

Theta range for data collection

Limiting indices

Reflections collected / unique

Completeness to theta $=28.00$
$\mathrm{C}_{75} \mathrm{H}_{58} \mathrm{~B} \mathrm{~F}_{24}$ Ir N O P

1679.20

293(2) K

$0.71073 \mathrm{~A}$

Trigonal, P3(1)

$\mathrm{a}=13.046(2) \mathrm{A}$

$b=13.046(2) A$

$\mathrm{c}=36.506(12) \mathrm{A}$

alpha $=90$ deg

beta $=90$ deg.

gamma $=120 \mathrm{deg}$.

5381(2) $A^{\wedge} 3$

3, $1.555 \mathrm{Mg} / \mathrm{m}^{\wedge} 3$

$1.991 \mathrm{~mm}^{\wedge}-1$

2508

$0.22 \times 0.18 \times 0.16 \mathrm{~mm}$

1.67 to $28.00 \mathrm{deg}$.

$-17<=\mathrm{h}<=14$

$-15<=\mathrm{k}<=16$

$-48<=\mathrm{l}<=45$

$99.8 \%$ 
Absorption correction

Max. and min. transmission

Refinement method

Data / restraints / parameters

Goodness-of-fit on $\mathrm{F}^{\wedge} 2$

Final R indices [I $>2 \operatorname{sigma}(\mathrm{I})]$

$\mathrm{R}$ indices (all data)

Absolute structure parameter

Largest diff. peak and hole
Semi-empirical from equivalents

1.000000 and 0.917897

Full-matrix least-squares on $\mathrm{F}^{\wedge} 2$

16043 / 457 / 1163

1.101

$\mathrm{R} 1=0.0529, \mathrm{wR} 2=0.1064$

$\mathrm{R} 1=0.0696, \mathrm{wR} 2=0.1119$

$0.003(5)$

0.673 and -1.933 e. $A^{\wedge}-3$

\section{Preparation of Imine Substrates}

General procedure: All of the imines were prepared from corresponding aniline and acetophenone according to literature methods ${ }^{3}$ with modification. The mixture of $\mathrm{NaHCO}_{3}$ (17 g, $0.2 \mathrm{~mol})$, aniline (50 mmol), acetophenone (50 mmol), $4 \AA$ molecular sieves and toluene $(40 \mathrm{~mL})$ in a two-necked round bottom bottle was heated at reflux under nitrogen atmosphere. The reaction was monitored with GC for maximum conversion. The reaction mixture was filtered through Celite, washed with $\mathrm{CH}_{2} \mathrm{Cl}_{2}$. The filtrate was collected, and the solvents were evaporated in vacuum. The product was distilled out and purified by recystallization with ethanol. The substrates $\mathbf{8 a},{ }^{3} \mathbf{8 b},{ }^{3} \mathbf{8 i},{ }^{4} \mathbf{8 k},{ }^{4}$ and $\mathbf{8 m}{ }^{4}$ were known compounds.

N-Phenyl-(1-(4-methylphenyl)ethylidene)amine (8c): Pale yellow solid, 52\% yield. Mp 57-59 ${ }^{\circ} \mathrm{C} ;{ }^{1} \mathrm{H}$ NMR (300 MHz, $\mathrm{CDCl}_{3}$ ) $\delta 7.92$ (d, $\left.J=8.1 \mathrm{~Hz}, 2 \mathrm{H}, \mathrm{Ar}-\mathrm{H}\right), 7.41-7.28$ (m, 4H, Ar-H), 7.15-7.09 (m, 1H, Ar-H), 6.83 (d, $J=8.4$ Hz, 2H, Ar-H), 2.45 (s, 3H, $\mathrm{CH}_{3}$ ), 2.25 (s, $\left.3 \mathrm{H}, \mathrm{CH}_{3}\right) ;{ }^{13} \mathrm{C}$ NMR $\left(75 \mathrm{MHz}, \mathrm{CDCl}_{3}\right) \delta$ 165.2, 152.0, 140.7, 136.9, 129.1, 129.0, 127.2, 123.1, 119.5, 21.4, 17.3; HRMS (EI) calcd for $\mathrm{C}_{15} \mathrm{H}_{15} \mathrm{~N}$ : 209.1204; Found 209.1199. 

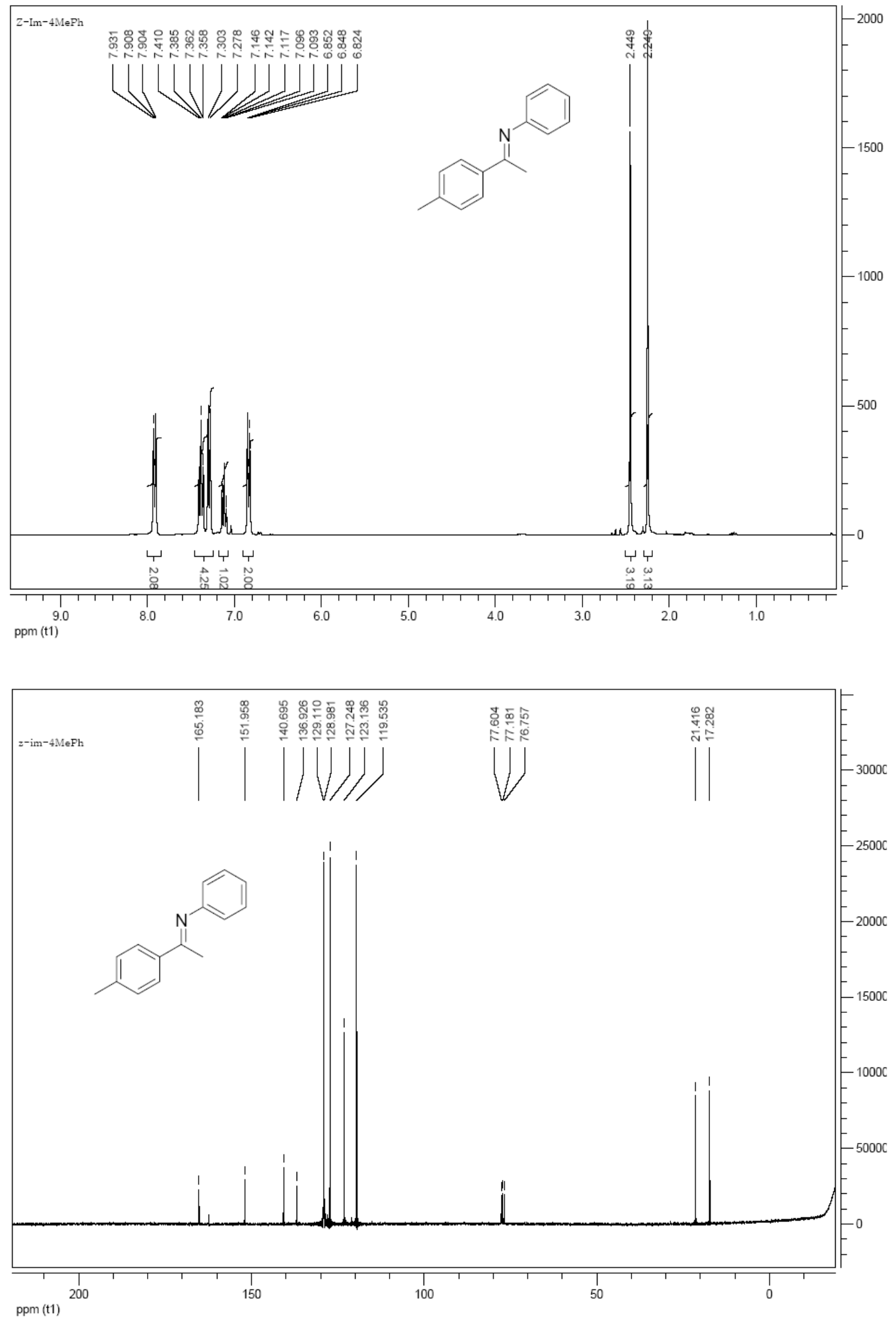

N-Phenyl-(1-(4-chlorophenyl)ethylidene)amine (8d): Pale yellow solid, 70\% yield. Mp 93-95 ${ }^{\circ} \mathrm{C}$; ${ }^{1} \mathrm{H}$ NMR (300 MHz, $\left.\mathrm{CDCl}_{3}\right) \delta 7.90$ (d, $\left.J=8.4 \mathrm{~Hz}, 2 \mathrm{H}, \mathrm{Ar}-\mathrm{H}\right), 7.40-7.31$ (m, 4H, Ar-H), 7.08 (t, $J=7.5 \mathrm{~Hz}, 1 \mathrm{H}, \mathrm{Ar}-\mathrm{H}), 6.78$ (d, $J=8.4 \mathrm{~Hz}, 2 \mathrm{H}, \mathrm{Ar}-\mathrm{H}), 2.19$ (s, 3H, CH3); ${ }^{13} \mathrm{C}$ NMR $\left(75 \mathrm{MHz}, \mathrm{CDCl}_{3}\right) \delta 164.2,151.4,137.9,136.6,129.0,128.6,123.4,119.4,17.2$; HRMS (EI) calcd for $\mathrm{C}_{14} \mathrm{H}_{12} \mathrm{NCl}$ : 229.0658; Found 229.0653. 


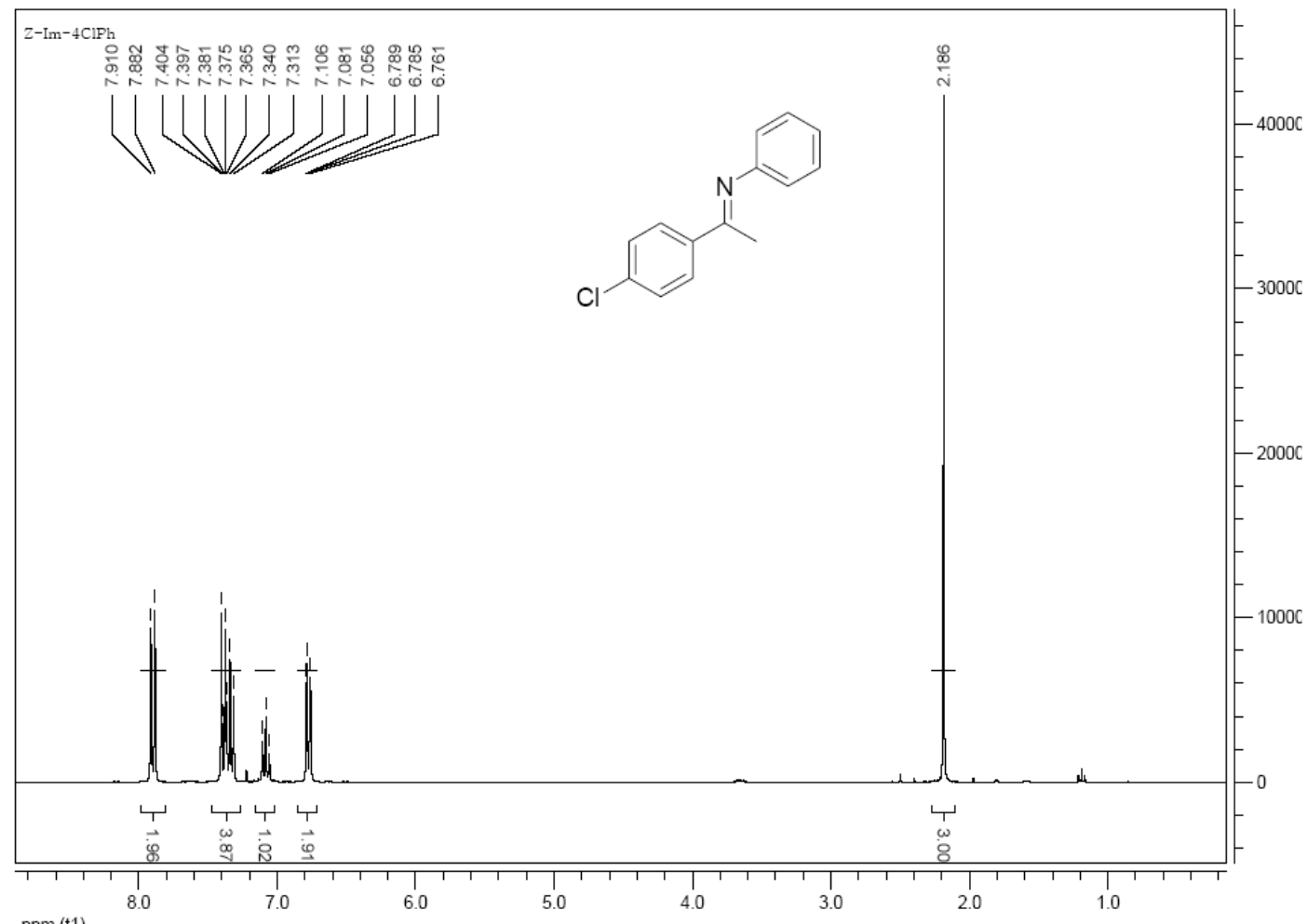

$\mathbf{N - P h e n y l - ( 1 - ( 4 - b r o m o p h e n y l ) e t h y l i d e n e ) a m i n e ~ ( 8 e ) : ~ Y e l l o w ~ s o l i d , ~ 4 5 \% ~ y i e l d . ~ M p ~ 8 1 - 8 2 ~}$ ${ }^{\circ} \mathrm{C}$; ${ }^{1} \mathrm{H}$ NMR (300 MHz, $\mathrm{CDCl}_{3}$ ) $\delta 7.81$ (d, $J=8.7 \mathrm{~Hz}, 2 \mathrm{H}, \mathrm{Ar}-\mathrm{H}$ ), 7.53 (d, $J=8.7 \mathrm{~Hz}, 2 \mathrm{H}$, Ar-H), 7.33 (t, $J=7.8 \mathrm{~Hz}, 2 \mathrm{H}, \mathrm{Ar}-\mathrm{H}), 7.07$ (t, $J=7.5 \mathrm{~Hz}, 1 \mathrm{H}, \mathrm{Ar}-\mathrm{H}$ ), 6.77 (d, $J=7.5 \mathrm{~Hz}, 2 \mathrm{H}$, Ar-H), 2.17 (s, 3H, CH3); ${ }^{13} \mathrm{C}$ NMR (75 MHz, $\left.\mathrm{CDCl}_{3}\right) \delta$ 164.3, 151.4, 138.4, 131.6, 129.1, 129.0, 128.9, 128.8, 125.1, 123.5, 119.4, 17.2; MS (ESI) m/z 274 (M, 100); Anal. calcd for $\mathrm{C}_{14} \mathrm{H}_{12} \mathrm{BrN}$ : C 61.33, H 4.41, N 5.11; Found: C 61.10, H 4.31, N 4.95. 

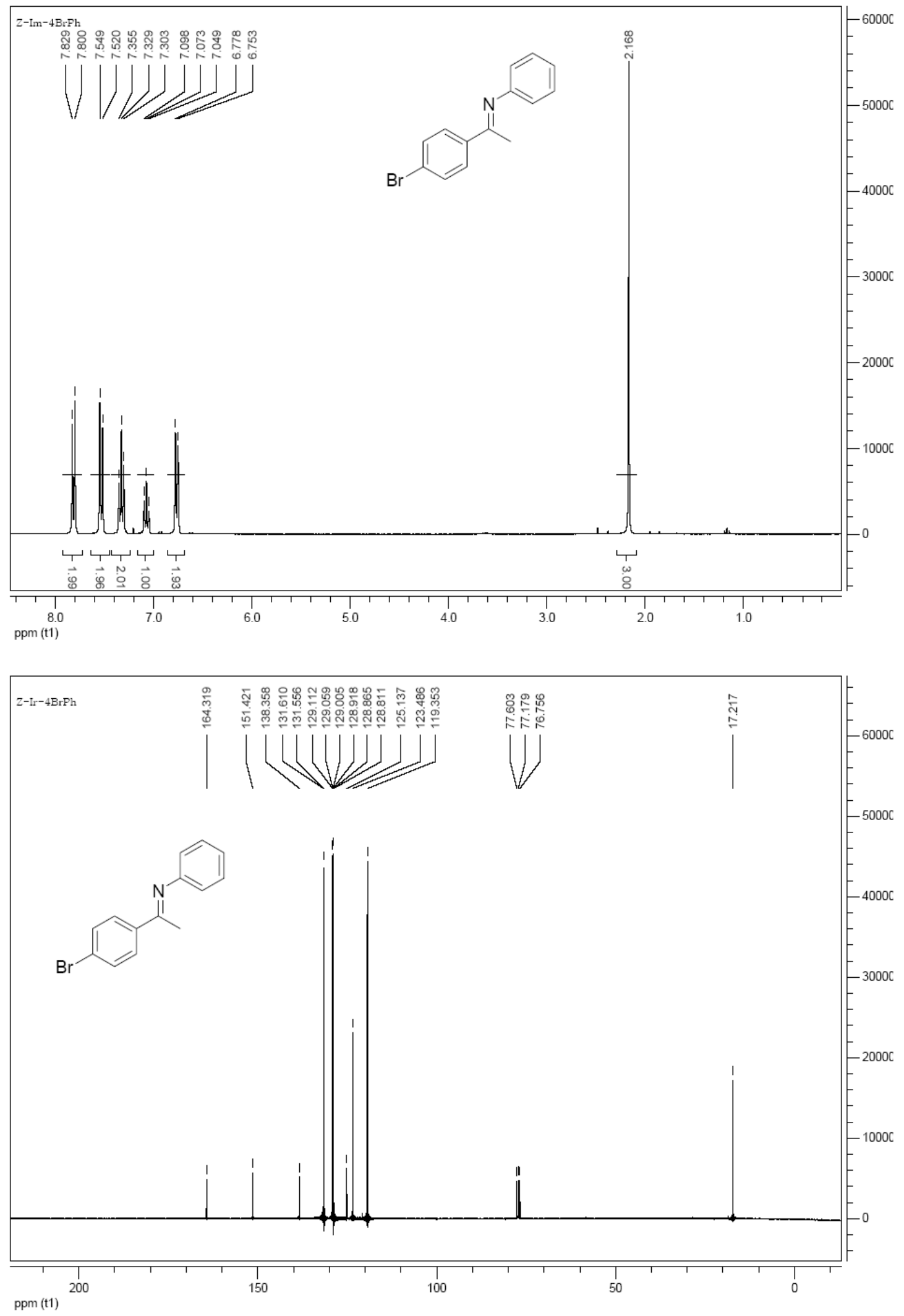

$\mathbf{N}$-Phenyl-(1-(3-chlorophenyl)ethylidene)amine (8f): Pale yellow solid, 65\% yield. Mp 50-51 ${ }^{\circ} \mathrm{C} ;{ }^{1} \mathrm{H}$ NMR (300 MHz, $\left.\mathrm{CDCl}_{3}\right) \delta$ 7.99-7.97 (m, 1H, Ar-H), 7.82-7.79 (m, 1H, Ar-H), 7.42-7.31 (m, 4H, Ar-H), 7.08 (t, $J=7.5 \mathrm{~Hz}, 1 \mathrm{H}, \mathrm{Ar}-\mathrm{H}), 6.78$ (d, $J=8.4 \mathrm{~Hz}, 2 \mathrm{H}$, Ar-H), 2.18 (s, 3H, $\left.\mathrm{CH}_{3}\right) ;{ }^{13} \mathrm{C}$ NMR $\left(75 \mathrm{MHz}, \mathrm{CDCl}_{3}\right) \delta$ 164.2, 151.3, 141.3, 134.6, 130.4, 129.6, 129.1, 129.0, 127.4, 125.4, 123.6, 119.3, 119.2, 17.3; MS (ESI) m/z 230 (M+1, 100); Anal. calcd for 
$\mathrm{C}_{14} \mathrm{H}_{12} \mathrm{ClN}$ : C 73.20, H 5.27, N 6.10; Found: C 73.20, H 5.28, N 6.13.

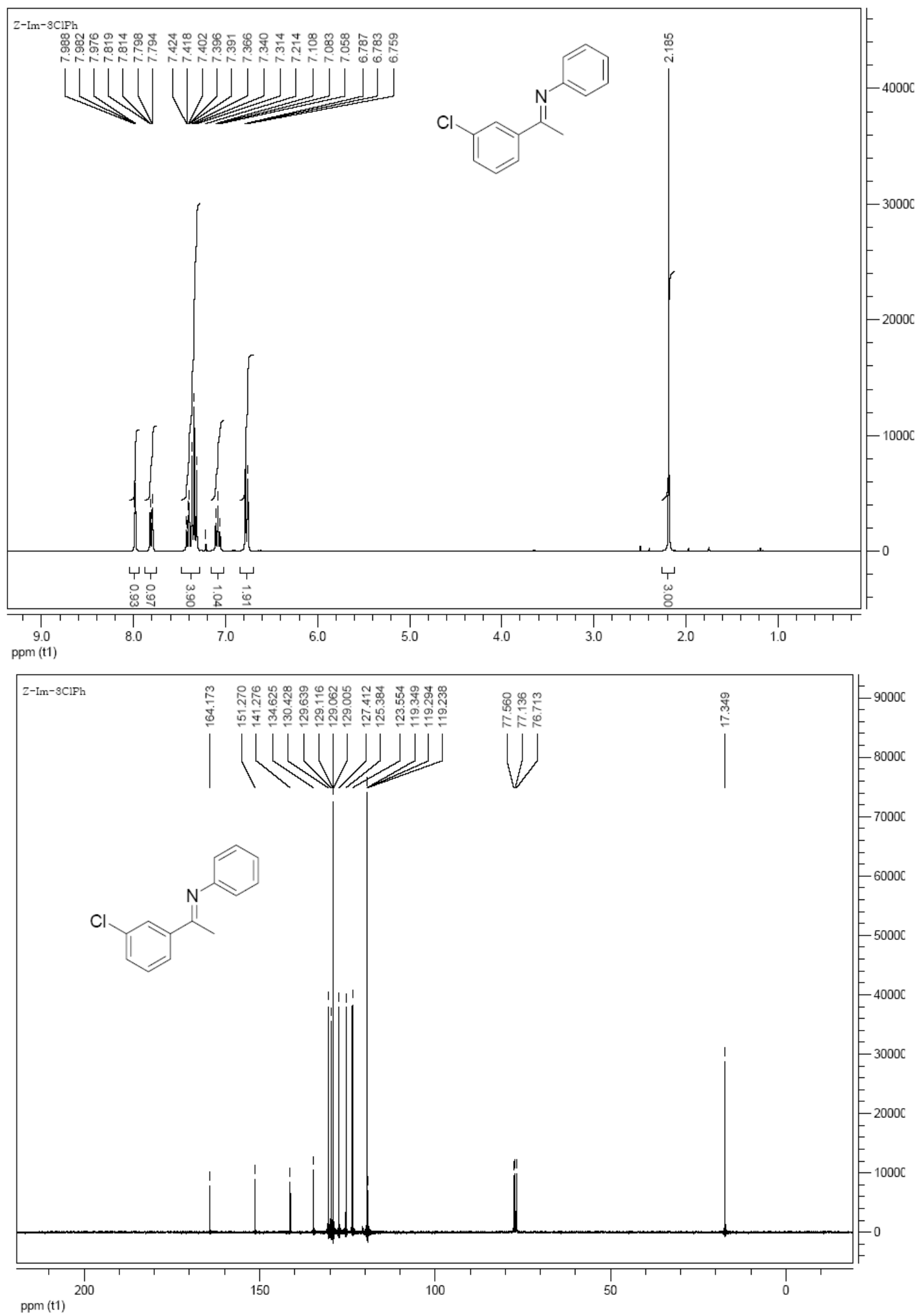

$\mathrm{N}$-Phenyl-(1-(3-bromophenyl)ethylidene)amine (8g): White solid, $67 \%$ yield. Mp $61-62{ }^{\circ} \mathrm{C}$; ${ }^{1} \mathrm{H}$ NMR (300 MHz, CDCl 3 ) $\delta 8.14$ (t, $\left.J=1.8 \mathrm{~Hz}, 1 \mathrm{H}, \mathrm{Ar}-\mathrm{H}\right), 7.85$ (d, $\left.J=7.8 \mathrm{~Hz}, 1 \mathrm{H}, \mathrm{Ar}-\mathrm{H}\right)$, 7.57 (d, $J=7.8 \mathrm{~Hz}, 1 \mathrm{H}$, Ar-H), 7.37-7.22 (m, 3H, Ar-H), 7.09 (t, $J=7.2 \mathrm{~Hz}, 1 \mathrm{H}$, Ar-H), 6.77 (d, $J=7.2 \mathrm{~Hz}, 2 \mathrm{H}, \mathrm{Ar}-\mathrm{H}), 2.19$ (s, 3H, $\left.\mathrm{CH}_{3}\right) ;{ }^{13} \mathrm{C} \mathrm{NMR}\left(75 \mathrm{MHz}, \mathrm{CDCl}_{3}\right.$ ) $\delta 164.1,151.2$, 141.5, 133.4, 130.3, 130.0, 129.1, 129.0, 125.8, 123.6; 122.8, 119.3, 119.2, 17.3; HRMS (EI) 
calcd for $\mathrm{C}_{14} \mathrm{H}_{12} \mathrm{NBr}$ : 273.0153; Found 273.0154.

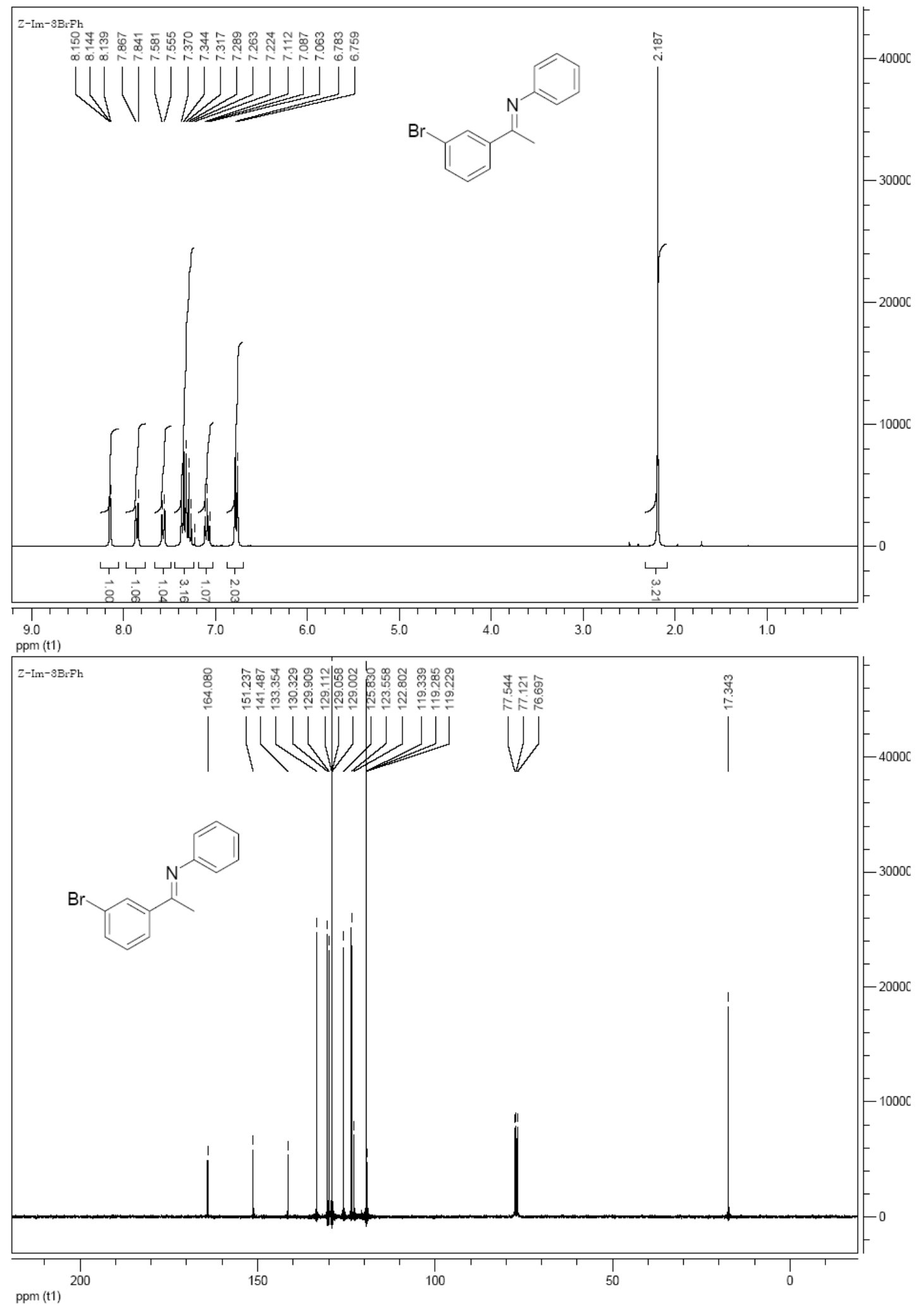

$\mathbf{N}$-Phenyl-(1-(3,4-dimethylphenyl)ethylidene)amine (8h): White solid, $78 \%$ yield. Mp 73-74 ${ }^{\circ} \mathrm{C} ;{ }^{1} \mathrm{H}$ NMR $\left(300 \mathrm{MHz}, \mathrm{CDCl}_{3}\right) \delta 7.79(\mathrm{~s}, 1 \mathrm{H}, \mathrm{Ar}-\mathrm{H}), 7.65$ (dd, $J=1.5$ and $7.8 \mathrm{~Hz}$, 1H, Ar-H), 7.32 (t, $J=8.1 \mathrm{~Hz}, 2 \mathrm{H}, \mathrm{Ar}-\mathrm{H}$ ), 7.18 (d, $J=8.1 \mathrm{~Hz}, 1 \mathrm{H}, \mathrm{Ar}-\mathrm{H}), 7.05$ (t, $J=7.5 \mathrm{~Hz}$, 1H, Ar-H), 6.78 (d, J = 7.5 Hz, 2H, Ar-H), 2.31 (s, 3H, $\mathrm{CH}_{3}$ ), 2.29 (s, 3H, $\mathrm{CH}_{3}$ ), 2.18 (s, 3H, $\left.\mathrm{CH}_{3}\right) ;{ }^{13} \mathrm{C}$ NMR $\left(75 \mathrm{MHz}, \mathrm{CDCl}_{3}\right) \delta 165.5,152.0,139.4,137.3,136.6,129.7,129.0,128.3$, 
125.0, 123.1, 119.6, 19.9, 19.8, 17.4; MS (ESI) m/z $224(\mathrm{M}+1,100)$; Anal. calcd for $\mathrm{C}_{16} \mathrm{H}_{17} \mathrm{~N}$ : C 86.05, H 7.67, N 6.27; Found: C 86.03, H 7.51, N 6.22.

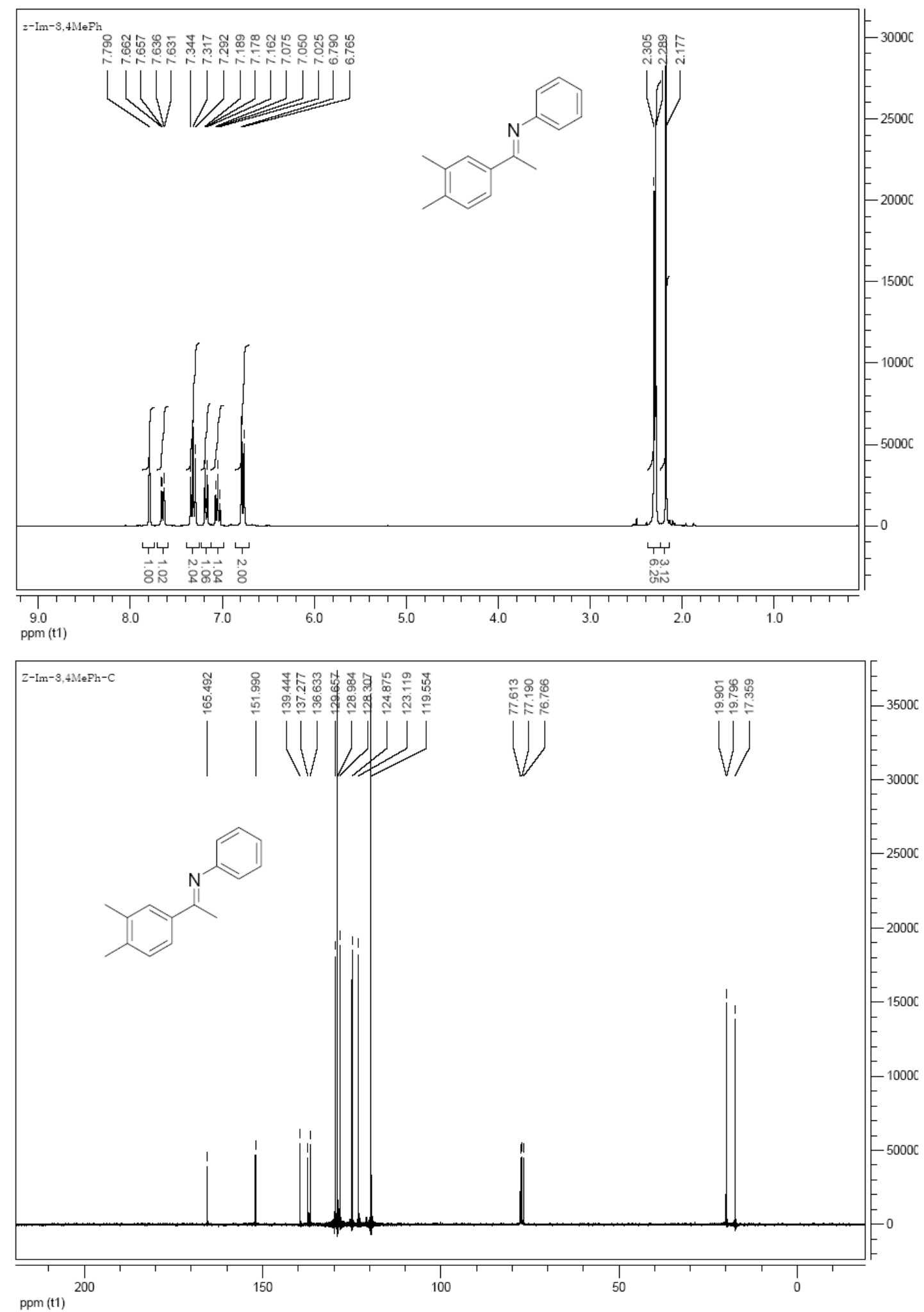

$\mathbf{N}$-(4-Chlorophenyl)-(1-phenylethylidene)amine (8j): Pale yellow solid, 70\% yield. Mp 78-79 ${ }^{\circ} \mathrm{C} ;{ }^{1} \mathrm{H}$ NMR (300 MHz, $\left.\mathrm{CDCl}_{3}\right) \delta$ 7.97-7.93 (m, 2H, Ar-H), 7.47-7.40 (m, 3H, Ar-H), 7.31-7.28 (m, 2H, Ar-H), 6.75-6.70 (m, 2H, Ar-H), 2.21 (s, 3H, $\left.\mathrm{CH}_{3}\right) ;{ }^{13} \mathrm{C}$ NMR (75 MHz, $\left.\mathrm{CDCl}_{3}\right) \delta 166.2,150.2,139.2,130.8,129.1,129.0,128.5,128.4,127.3,127.2,120.9,120.8$, 
17.4; HRMS (EI) calcd for $\mathrm{C}_{14} \mathrm{H}_{12} \mathrm{NCl}$ : 229.0658; Found 229.0658.

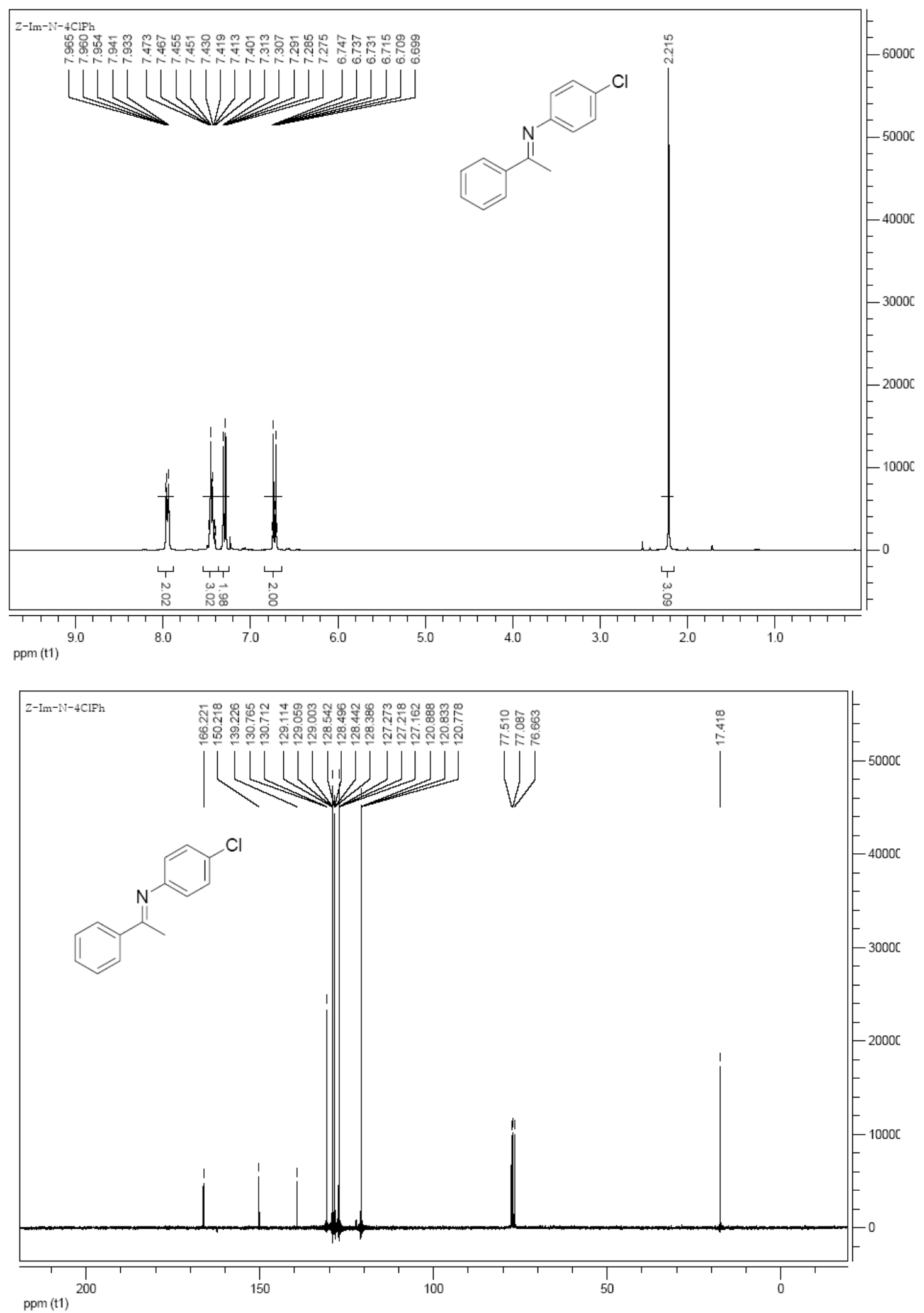

$\mathbf{N}$-(3-Methylphenyl)-(1-phenylethylidene)amine (81): Yellow solid, 65\% yield. Mp 27-28 ${ }^{0} \mathrm{C}$; ${ }^{1} \mathrm{H}$ NMR (300 MHz, $\mathrm{CDCl}_{3}$ ) $\delta$ 7.97-7.94 (m, 2H, Ar-H), 7.43-7.39 (m, 3H, Ar-H), 7.22 (t, $\mathrm{J}=7.5 \mathrm{~Hz}, 1 \mathrm{H}, \mathrm{Ar}-\mathrm{H}), 6.88$ (d, J = 7.5 Hz, 1H, Ar-H), 6.61-6.57 (m, 2H, Ar-H), 2.34 (s, 3H, $\mathrm{CH}_{3}$ ), 2.21 (s, 3H, $\mathrm{CH}_{3}$ ); ${ }^{13} \mathrm{C}$ NMR $\left(75 \mathrm{MHz}, \mathrm{CDCl}_{3}\right.$ ) $\delta 165.2,151.8,139.6,138.8,130.4$, 
128.9, 128.4, 127.3, 127.2, 124.0, 120.0, 116.4, 21.5, 17.4; MS (ESI) m/z 210 (M+1, 100); Anal. calcd for $\mathrm{C}_{15} \mathrm{H}_{15} \mathrm{~N}$ : C 86.08, H 7.22, N 6.69; Found: C 85.89, H 7.28, N 6.73.

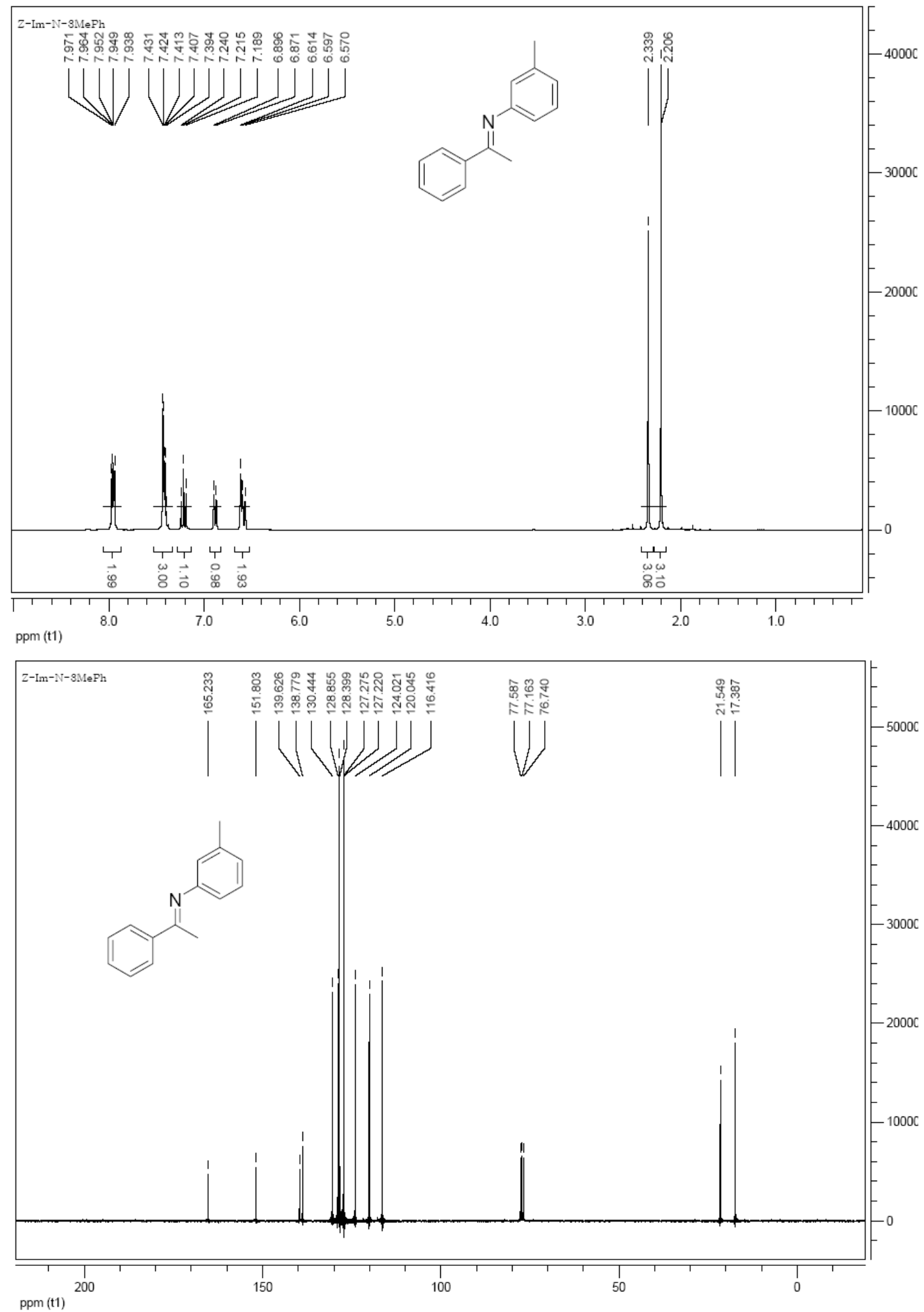



Products

(R)- $\mathbf{N}$-Phenyl-1-phenylethylamine (9a): ${ }^{5}$ Colorless oil. $[\alpha]^{18}{ }_{\mathrm{D}}-37\left(c 0.91, \mathrm{CH}_{2} \mathrm{Cl}_{2}\right)$; $[\alpha]^{18}$ -14.7 (c 0.505, MeOH); ${ }^{1} \mathrm{H}$ NMR (300 MHz, CDCl 3 ) $\delta$ 7.39-7.06 (m, 7H, Ar-H), 6.64 (t, $J=$ $7.2 \mathrm{~Hz}, 1 \mathrm{H}, \mathrm{Ar}-\mathrm{H}$ ), 6.50 (d, $J=7.5 \mathrm{~Hz}, 2 \mathrm{H}, \mathrm{Ar}-\mathrm{H}$ ), 4.48 (dd, $J=6.6$ and $13.5 \mathrm{~Hz}, 1 \mathrm{H}, \mathrm{CH}$ ), 4.03 (bs, $1 \mathrm{H}, \mathrm{NH}$ ), 1.51 (d, $J=6.9 \mathrm{~Hz}, 3 \mathrm{H}, \mathrm{CH}_{3}$ ). Conversion was determined by GC using HP-5 column (programmed at $5{ }^{\circ} \mathrm{C} / \mathrm{min}$ from $100{ }^{\circ} \mathrm{C}$ to $220{ }^{\circ} \mathrm{C}$ ), $t_{\mathrm{R}}=14.77$ (product) and 15.22 (substrate) min. Enantiomer ratio was determined by HPLC using a Chiralcel OD-H column (flow rate $1.0 \mathrm{~mL} / \mathrm{min}, n-\mathrm{Hex} / \mathrm{iPrOH} 98: 2, \lambda=254 \mathrm{~nm}$ ), $t_{\mathrm{R}}=14.91 \mathrm{~min}\left(\right.$ minor), $t_{\mathrm{R}}=$ $19.10 \mathrm{~min}$ (major); 93\% ee.

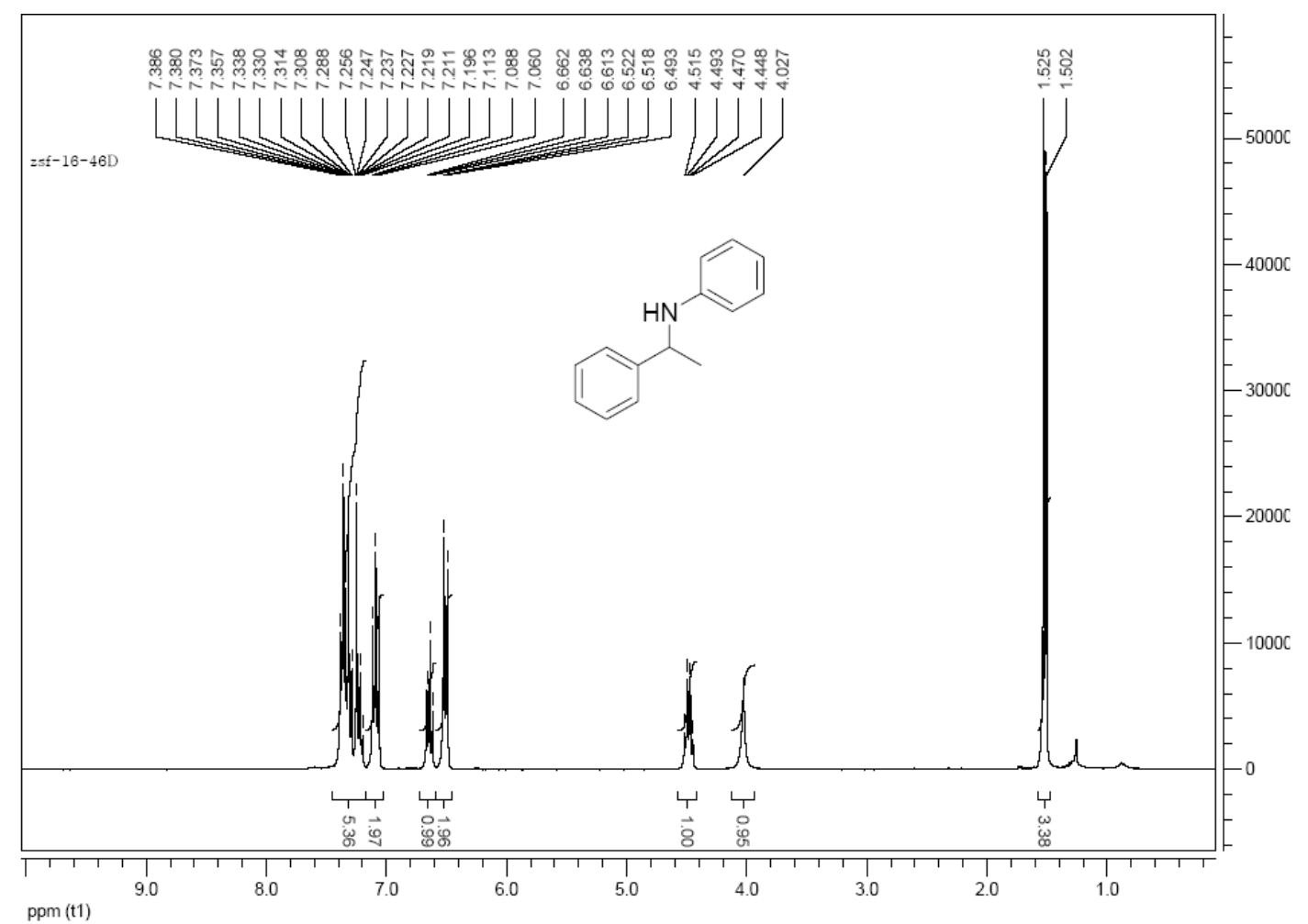



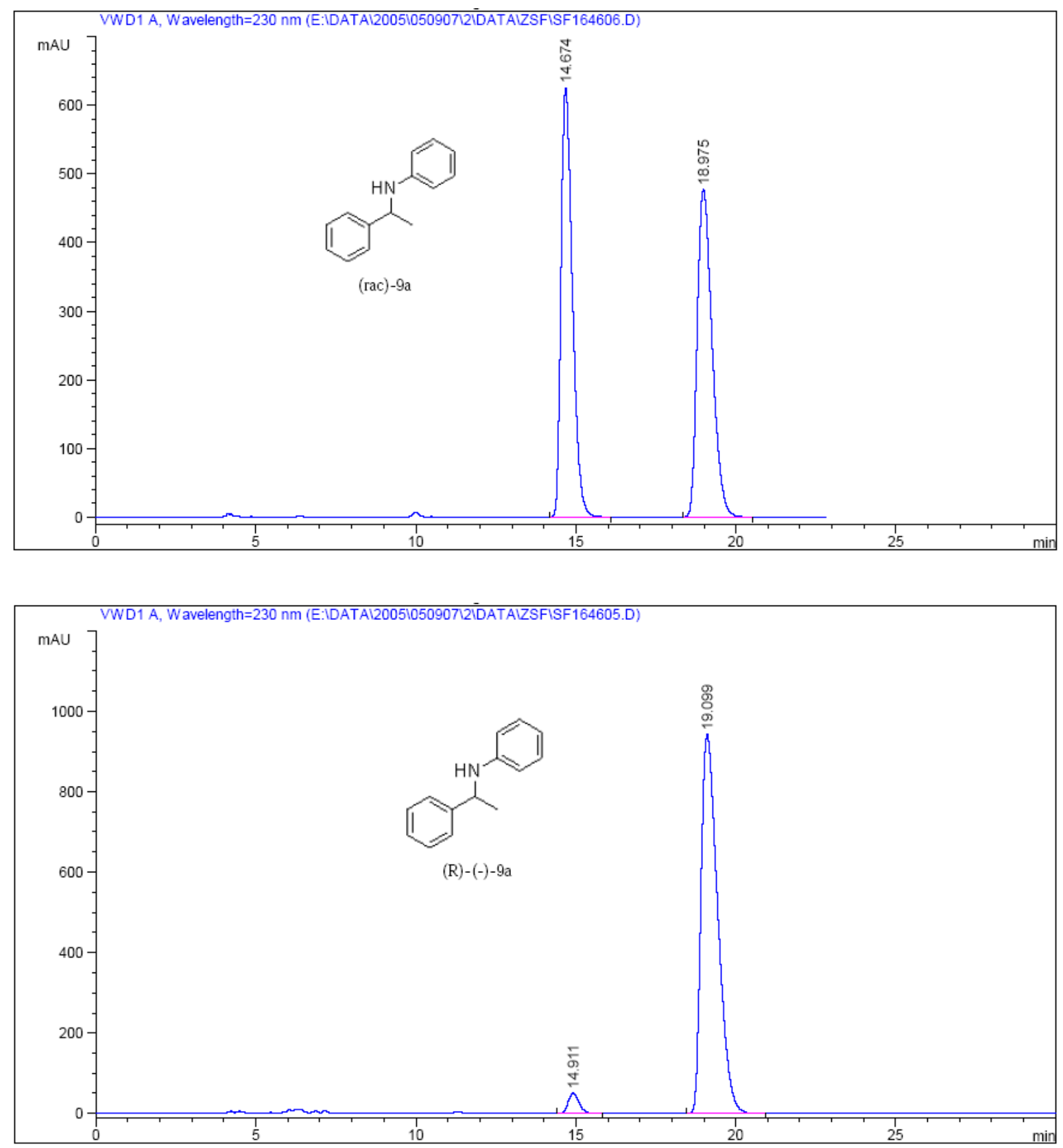

(-)- $N$-Phenyl-1-(4-methoxyphenyl)ethylamine (9b): ${ }^{3}$ White solid. Mp $76-79{ }^{\circ} \mathrm{C} ;[\alpha]^{18}-17$ (c 1.09, $\mathrm{CH}_{2} \mathrm{Cl}_{2}$ ); ${ }^{1} \mathrm{H}$ NMR (300 MHz, $\left.\mathrm{CDCl}_{3}\right) \delta$ 7.30-7.25 (m, 2H, Ar-H), 7.12-7.06 (m, 2H, Ar-H), 6.88-6.66 (m, 2H, Ar-H), 6.64 (t, $J=7.2 \mathrm{~Hz}, 1 \mathrm{H}, \mathrm{Ar}-\mathrm{H}), 6.50$ (d, $J=7.8 \mathrm{~Hz}, 2 \mathrm{H}$, Ar-H), 4.44 (dd, $J=6.6$ and 13.5 Hz, 1H, CH), 3.98 (bs, 1H, NH), 3.78 (s, 3H, $\mathrm{CH}_{3}$ ), 1.49 (s, $J=6.6 \mathrm{~Hz}, 3 \mathrm{H}, \mathrm{CH}_{3}$ ). Conversion was determined by GC using HP-5 column (programmed at $5{ }^{\circ} \mathrm{C} / \mathrm{min}$ from $100{ }^{\circ} \mathrm{C}$ to $220{ }^{\circ} \mathrm{C}$ ), $t_{\mathrm{R}}=19.19$ (product) and 20.17 (substrate) min. Enantiomer ratio was determined by HPLC using a Chiralcel OD-H column (flow rate 1.0 $\mathrm{mL} / \mathrm{min}, n-\mathrm{Hex} / \mathrm{iPrOH} 98: 2, \lambda=254 \mathrm{~nm}$ ), $t_{\mathrm{R}}=19.38 \mathrm{~min}$ (minor), $t_{\mathrm{R}}=22.16 \mathrm{~min}$ (major); 94\% ee. 

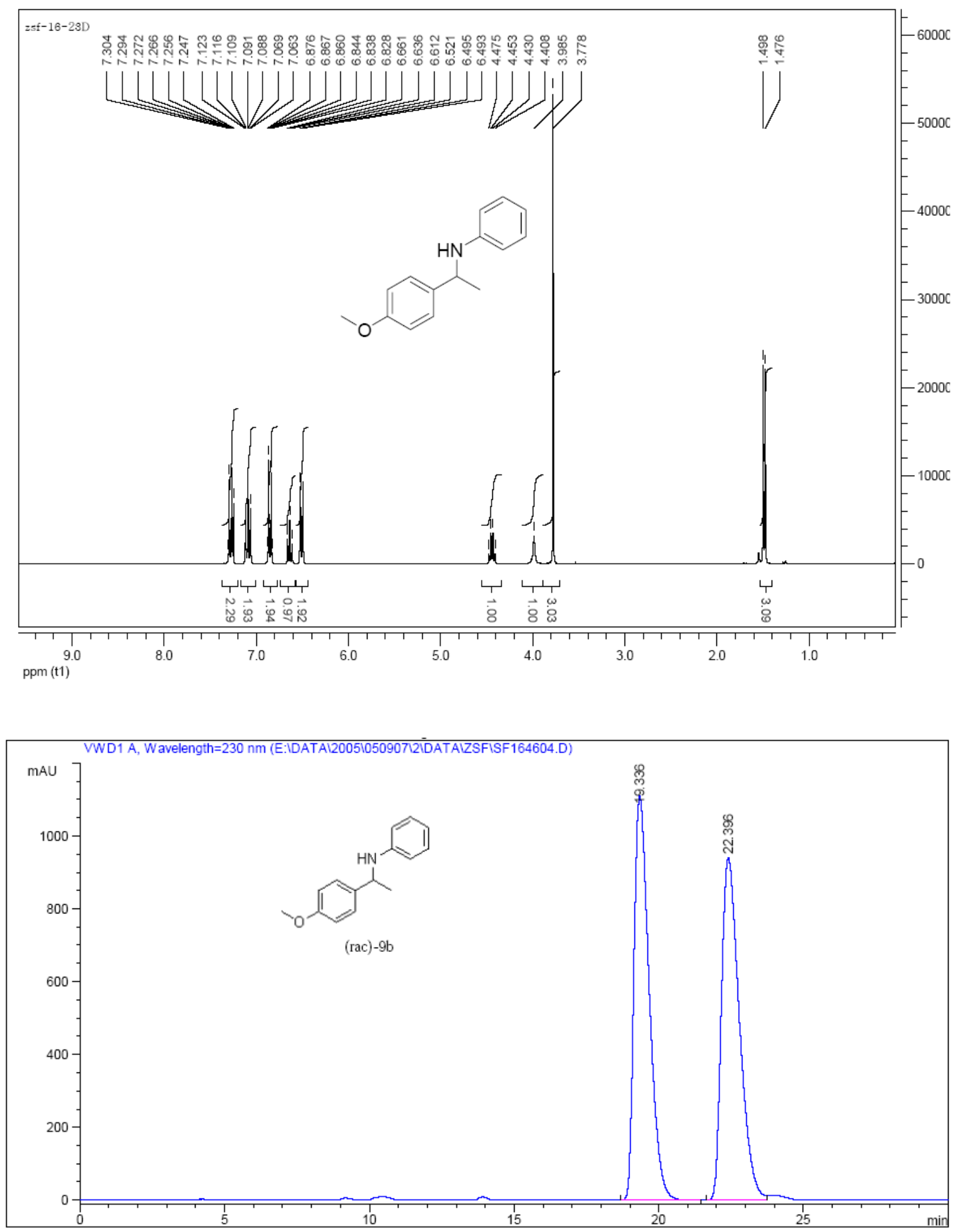


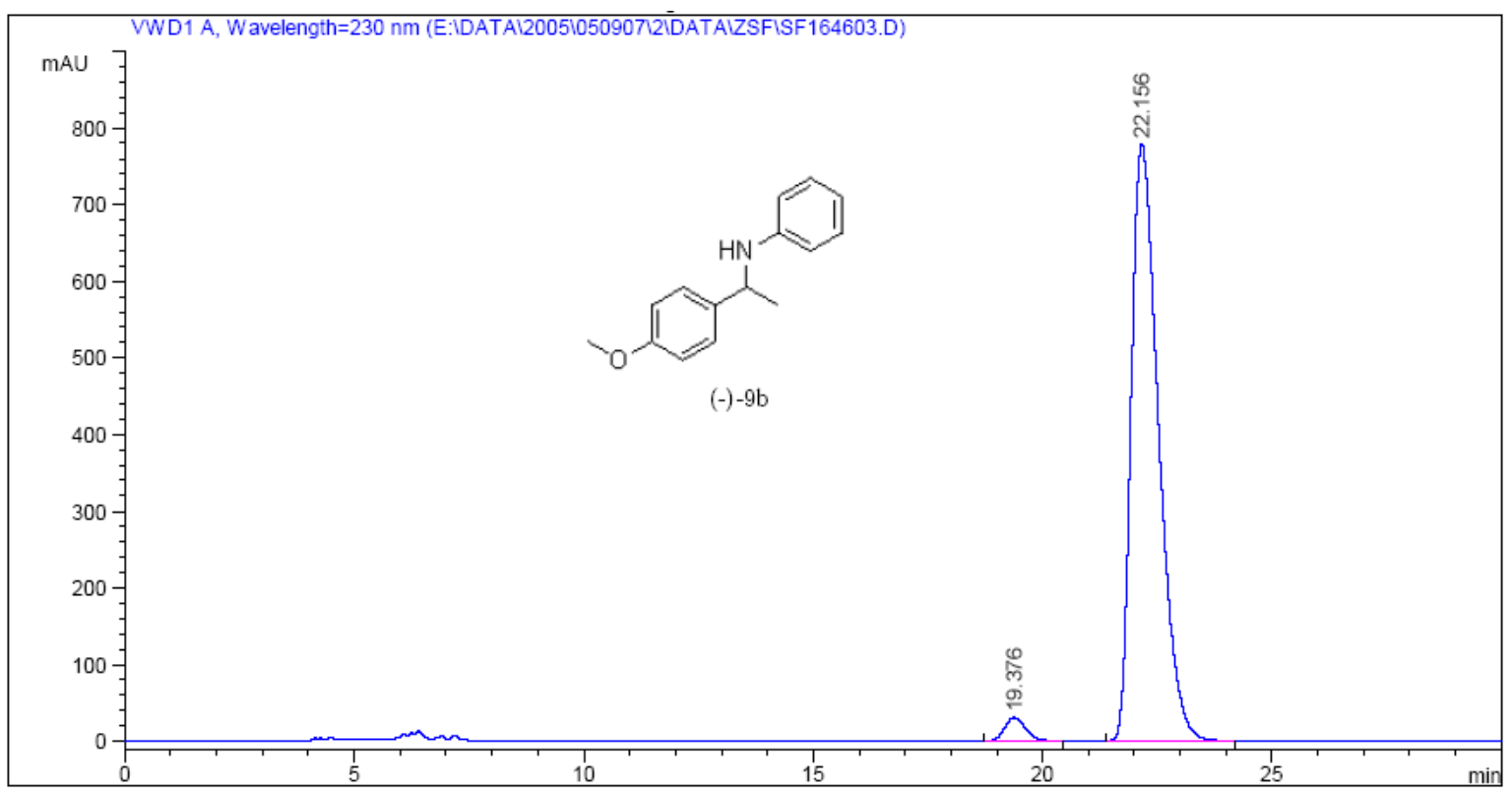

(-)- $\mathrm{N}$-Phenyl-1-(4-methylphenyl)ethylamine (9c): ${ }^{6}$ Colorless oil. $[\alpha]^{18}{ }_{\mathrm{D}}-18(c)$ $\mathrm{CH}_{2} \mathrm{Cl}_{2}$ ); ${ }^{1} \mathrm{H}$ NMR $\left(300 \mathrm{MHz}, \mathrm{CDCl}_{3}\right) \delta 7.25$ (d, $\left.J=7.5 \mathrm{~Hz}, 2 \mathrm{H}, \mathrm{Ar}-\mathrm{H}\right), 7.13-7.06(\mathrm{~m}, 4 \mathrm{H}$, Ar-H), 6.63 (t, $J=7.2 \mathrm{~Hz}, 1 \mathrm{H}, \mathrm{Ar}-\mathrm{H}), 6.51(\mathrm{~d}, J=7.8 \mathrm{~Hz}, 2 \mathrm{H}, \mathrm{Ar}-\mathrm{H}), 4.45$ (dd, $J=6.9$ and $13.5 \mathrm{~Hz}, 1 \mathrm{H}, \mathrm{CH}$ ), 4.00 (bs, $1 \mathrm{H}, \mathrm{NH}$ ), 2.32 (s, 3H, $\left.\mathrm{CH}_{3}\right), 1.49$ (d, J = $6.9 \mathrm{~Hz}, 3 \mathrm{H}, \mathrm{CH}_{3}$ ). Conversion was determined by GC using HP-5 column (programmed at $5{ }^{\circ} \mathrm{C} / \mathrm{min}$ from 100 ${ }^{\circ} \mathrm{C}$ to $220{ }^{\circ} \mathrm{C}$ ), $t_{\mathrm{R}}=16.14$ (product) and 16.93 (substrate) min. Enantiomer ratio was determined by HPLC using a Chiralcel OD-H column (flow rate $1.0 \mathrm{~mL} / \mathrm{min}, n-\mathrm{Hex} / \mathrm{iPrOH}$ 98:2, $\lambda=254 \mathrm{~nm}$ ), $t_{\mathrm{R}}=12.13 \mathrm{~min}$ (minor), $t_{\mathrm{R}}=14.54 \mathrm{~min}$ (major); $94 \%$ ee.

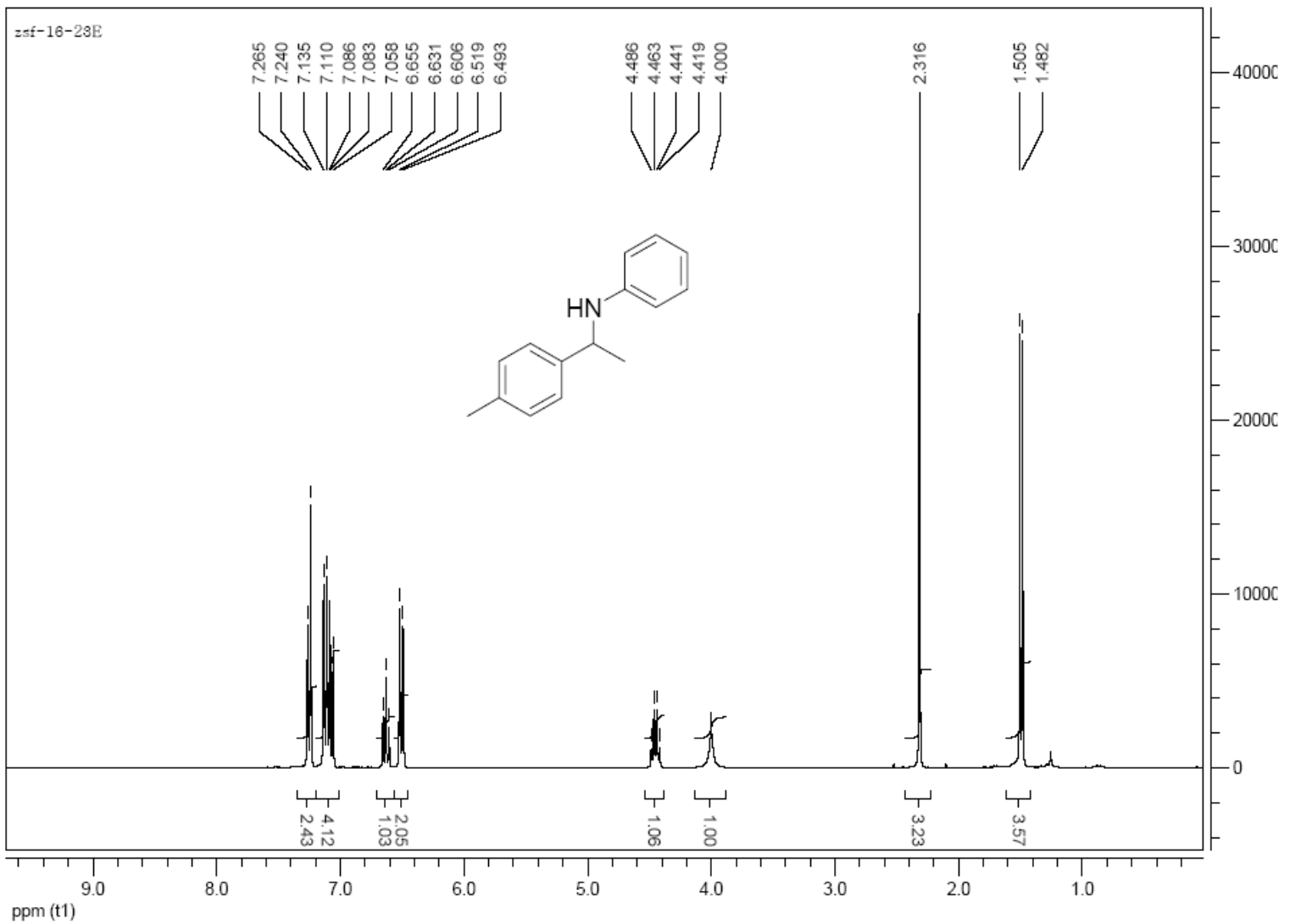



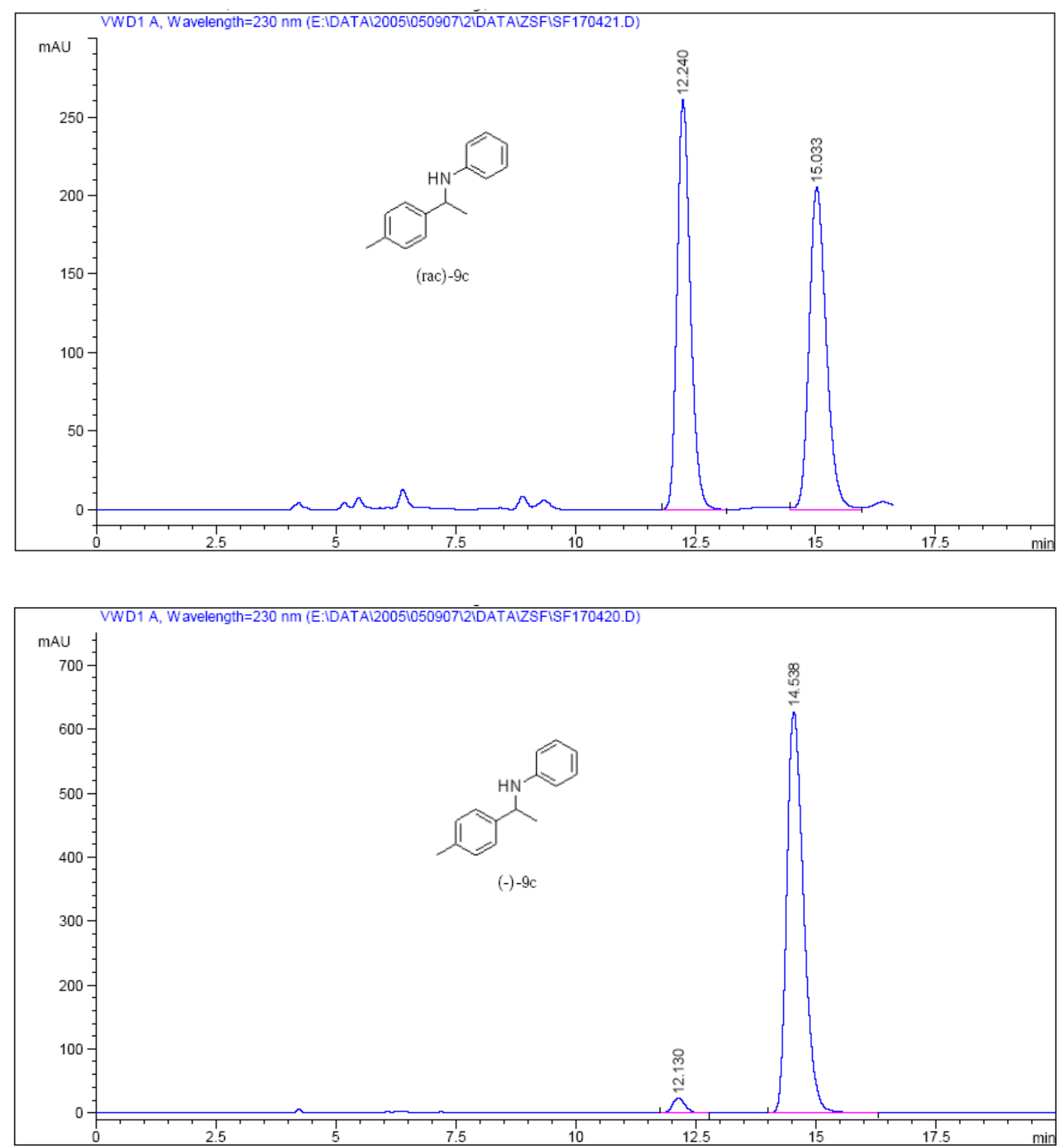

(-)-N-Phenyl-1-(4-chlorophenyl)ethylamine (9d): ${ }^{6}$ White solid. Mp $67-69{ }^{\circ} \mathrm{C}$; $[\alpha]^{18}{ }_{\mathrm{D}}-17(\mathrm{C}$ 1.7, $\mathrm{CH}_{2} \mathrm{Cl}_{2}$ ); ${ }^{1} \mathrm{H}$ NMR (300 MHz, $\mathrm{CDCl}_{3}$ ) $\delta 7.32-7.24(\mathrm{~m}, 4 \mathrm{H}, \mathrm{Ar}-\mathrm{H}), 7.11-7.06(\mathrm{~m}, 2 \mathrm{H}$, Ar-H), 6.65 (t, $J=7.2 \mathrm{~Hz}, 1 \mathrm{H}, \mathrm{Ar}-\mathrm{H}), 6.47$ (d, $J=7.8 \mathrm{~Hz}, 2 \mathrm{H}, \mathrm{Ar}-\mathrm{H}), 4.44$ (dd, $J=6.6$ and $13.2 \mathrm{~Hz}, 1 \mathrm{H}, \mathrm{CH}$ ), 4.00 (bs, $1 \mathrm{H}, \mathrm{NH}), 1.48$ (d, $J=6.9 \mathrm{~Hz}, 3 \mathrm{H}, \mathrm{CH}_{3}$ ). Conversion was determined by GC using HP-5 column (programmed at $5{ }^{\circ} \mathrm{C} / \mathrm{min}$ from $100{ }^{\circ} \mathrm{C}$ to $220{ }^{\circ} \mathrm{C}$ ), $t_{\mathrm{R}}=$ 18.17 (product) and 18.43 (substrate) min. Enantiomer ratio was determined by HPLC using a Chiralcel OD-H column (flow rate $1.0 \mathrm{~mL} / \mathrm{min}, n-\mathrm{Hex} / \mathrm{iPrOH} 98: 2, \lambda=254 \mathrm{~nm}$ ), $t_{\mathrm{R}}=21.69$ $\min (\operatorname{minor}), t_{\mathrm{R}}=27.49 \min$ (major); $90 \%$ ee. 


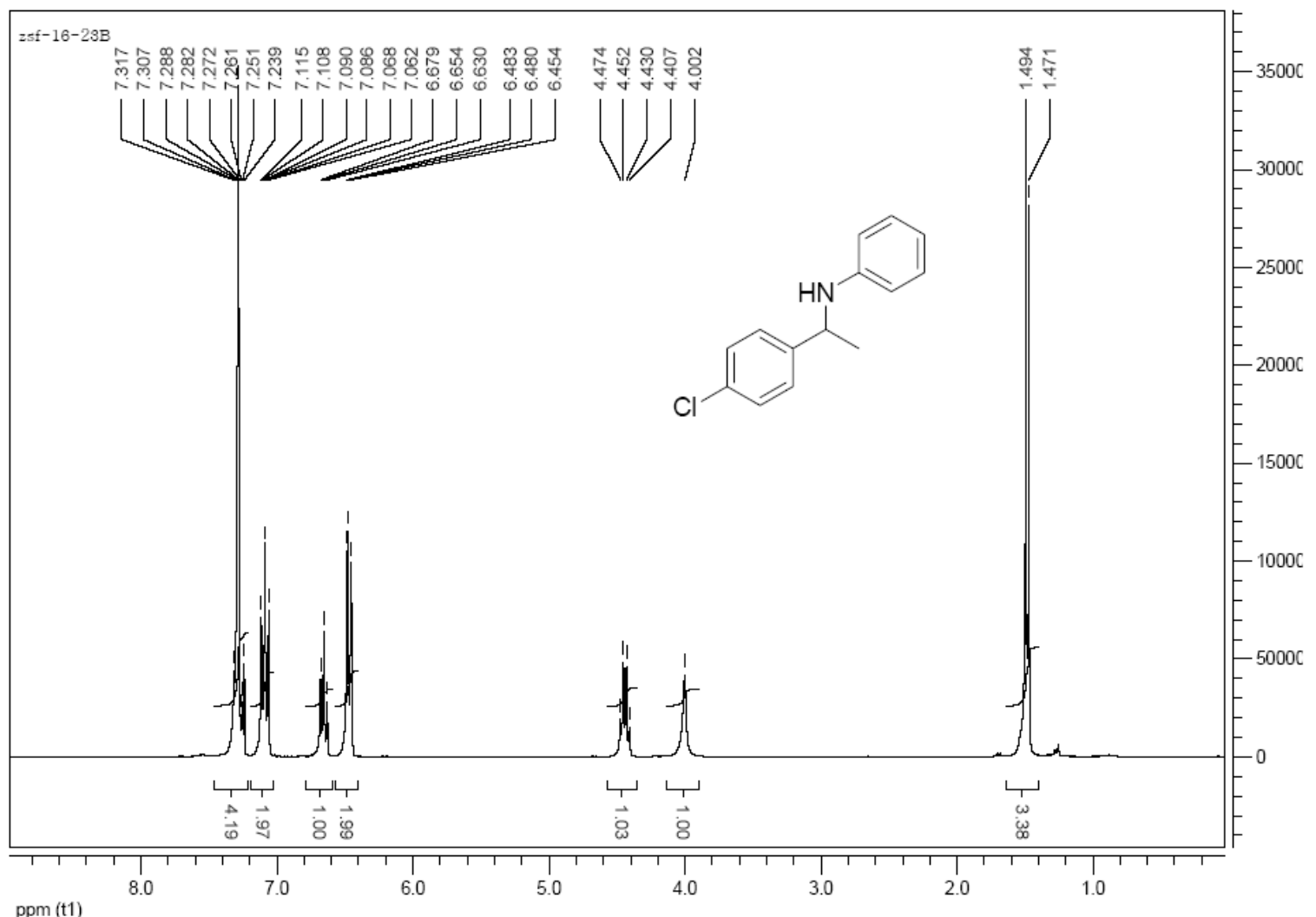

ppm (t1)

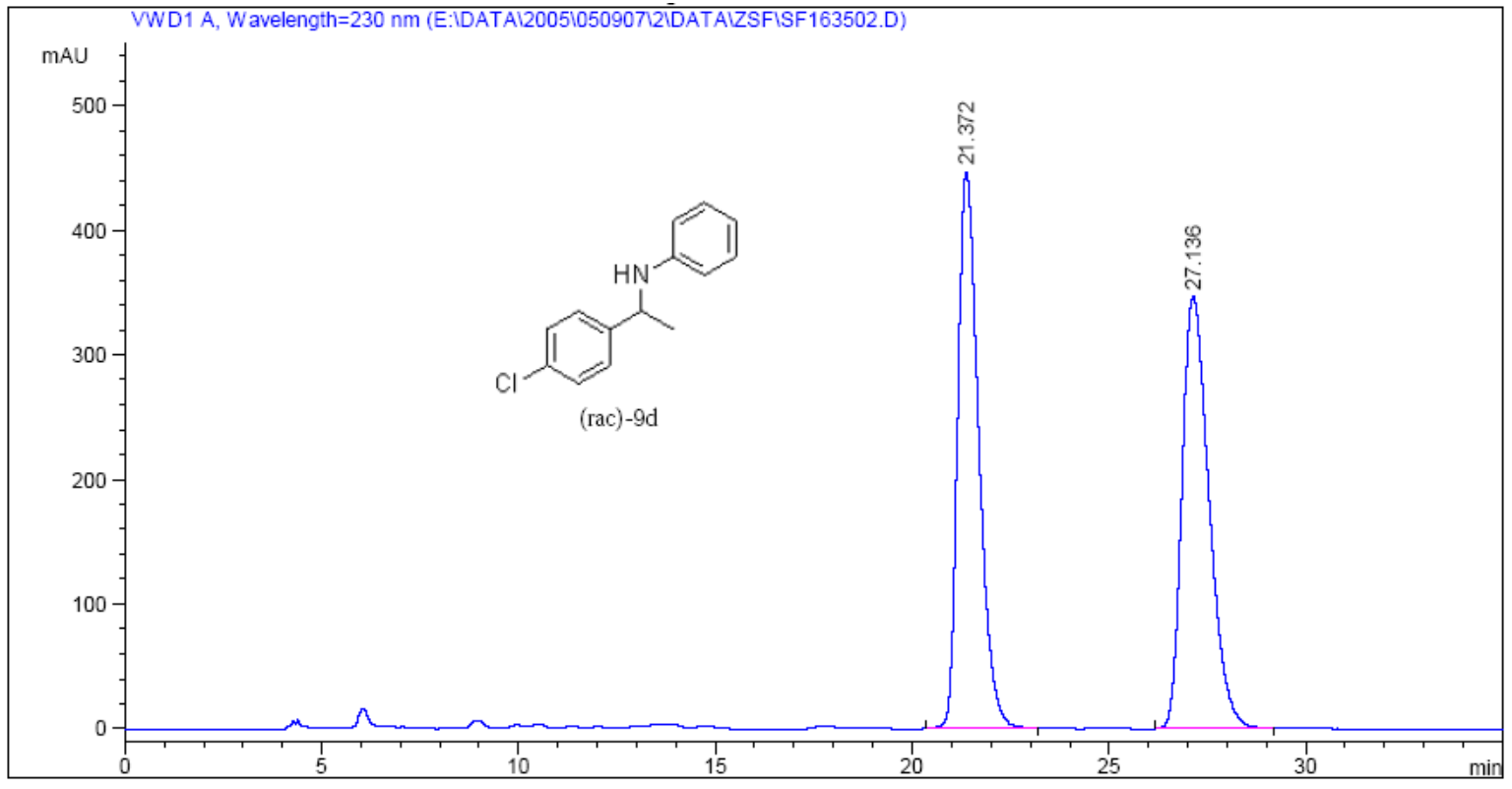




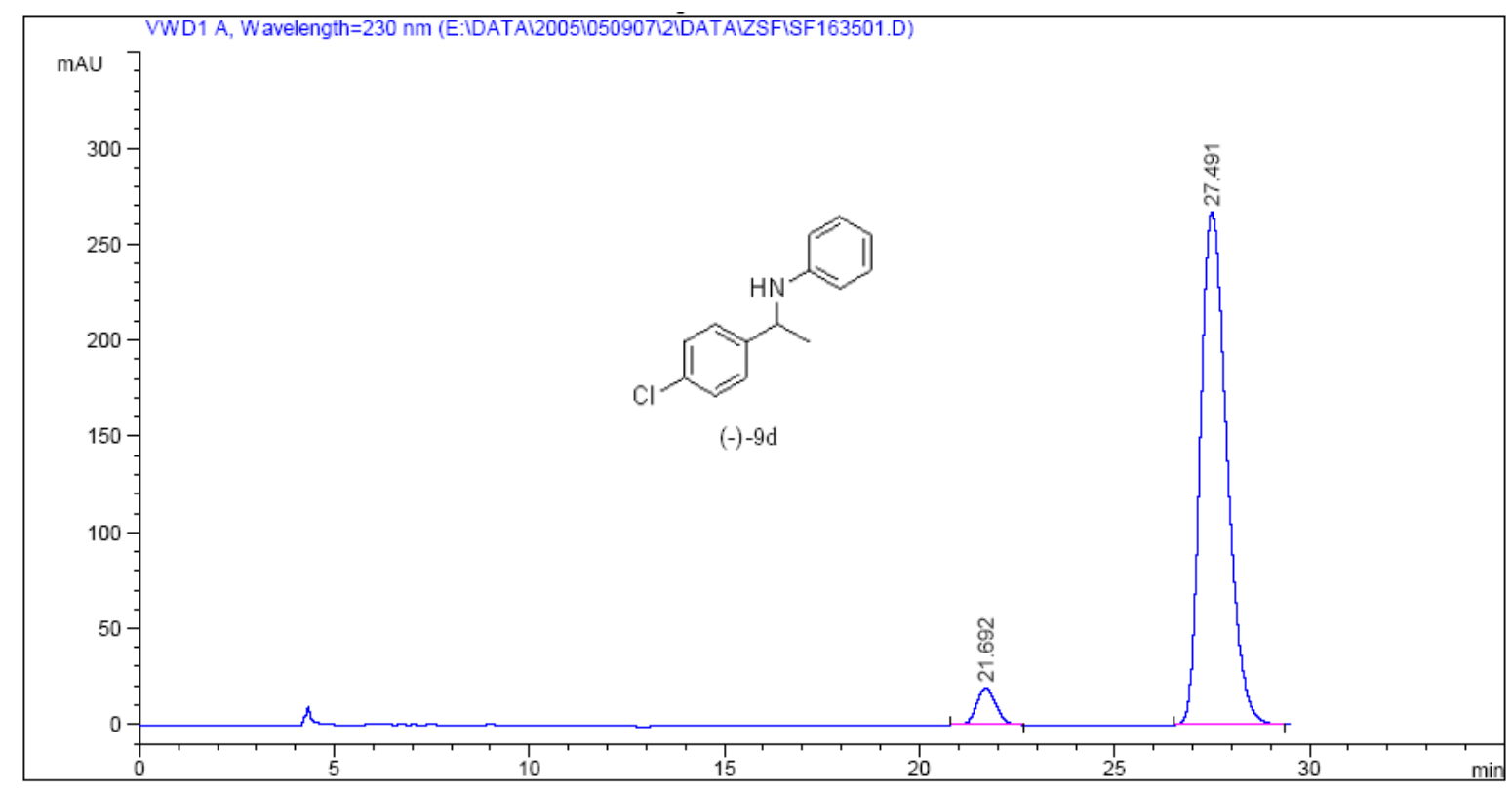

(R)-(-)- $\boldsymbol{N}$-Phenyl-1-(4-bromophenyl)ethylamine (9e): White solid. Mp $93-95{ }^{\circ} \mathrm{C} ;[\alpha]^{18}{ }_{\mathrm{D}}-8$ (c 1.39, $\mathrm{CH}_{2} \mathrm{Cl}_{2}$ ); ${ }^{1} \mathrm{H}$ NMR (300 MHz, $\mathrm{CDCl}_{3}$ ) $\delta$ 7.44-7.41 (m, 2H, Ar-H), 7.25-7.22 (m, 2H, Ar-H), 7.12-7.06 (m, 2H, Ar-H), 6.65 (t, $J=7.2 \mathrm{~Hz}, 1 \mathrm{H}, \mathrm{Ar}-\mathrm{H}), 6.46$ (d, J = $7.5 \mathrm{~Hz}, 2 \mathrm{H}$, Ar-H), 4.42 (dd, $J=6.6$ and $13.2 \mathrm{~Hz}, 1 \mathrm{H}, \mathrm{Ar}-\mathrm{H}$ ), 3.99 (bs, 1H, NH), 1.47 (d, J = 6.9 Hz, 3H, $\left.\mathrm{CH}_{3}\right) ;{ }^{13} \mathrm{C}$ NMR $\left(75 \mathrm{MHz}, \mathrm{CDCl}_{3}\right) \delta$ 147.0, 144.4, 131.8, 131.7, 129.2, 129.1, 127.7, 127.6, 120.5, 117.6, 113.4, 113.3, 53.1, 25.1; MS (ESI) m/z 276 (M, 100); Anal. calcd for $\mathrm{C}_{14} \mathrm{H}_{14} \mathrm{BrN}$ : C 60.89, H 5.11, N 5.07; Found: C 60.63, H 4.98, N 4.90. Conversion was determined by GC using HP-5 column (programmed at $5{ }^{\circ} \mathrm{C} / \mathrm{min}$ from $100{ }^{\circ} \mathrm{C}$ to $220{ }^{\circ} \mathrm{C}$ ), $t_{\mathrm{R}}=$ 16.95 (product) and 17.10 (substrate) min. Enantiomer ratio was determined by HPLC using a Chiralcel OD-H column (flow rate $1.0 \mathrm{~mL} / \mathrm{min}, n$-Hex/iPrOH 98:2, $\lambda=254 \mathrm{~nm}$ ), $t_{\mathrm{R}}=26.38$ $\min (\operatorname{minor}), t_{\mathrm{R}}=32.60 \mathrm{~min}$ (major); 91\% ee. 


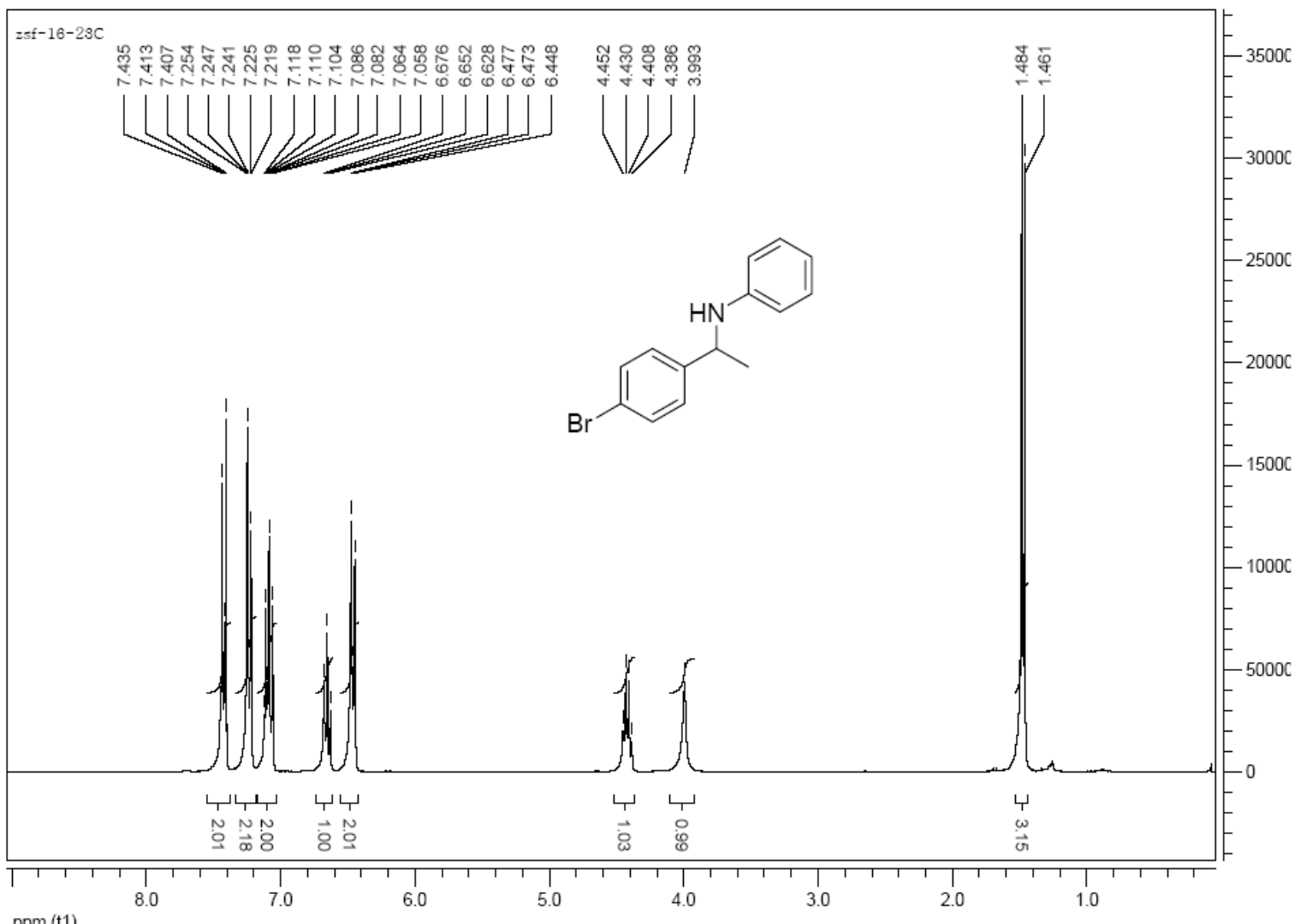

ppm (t1)

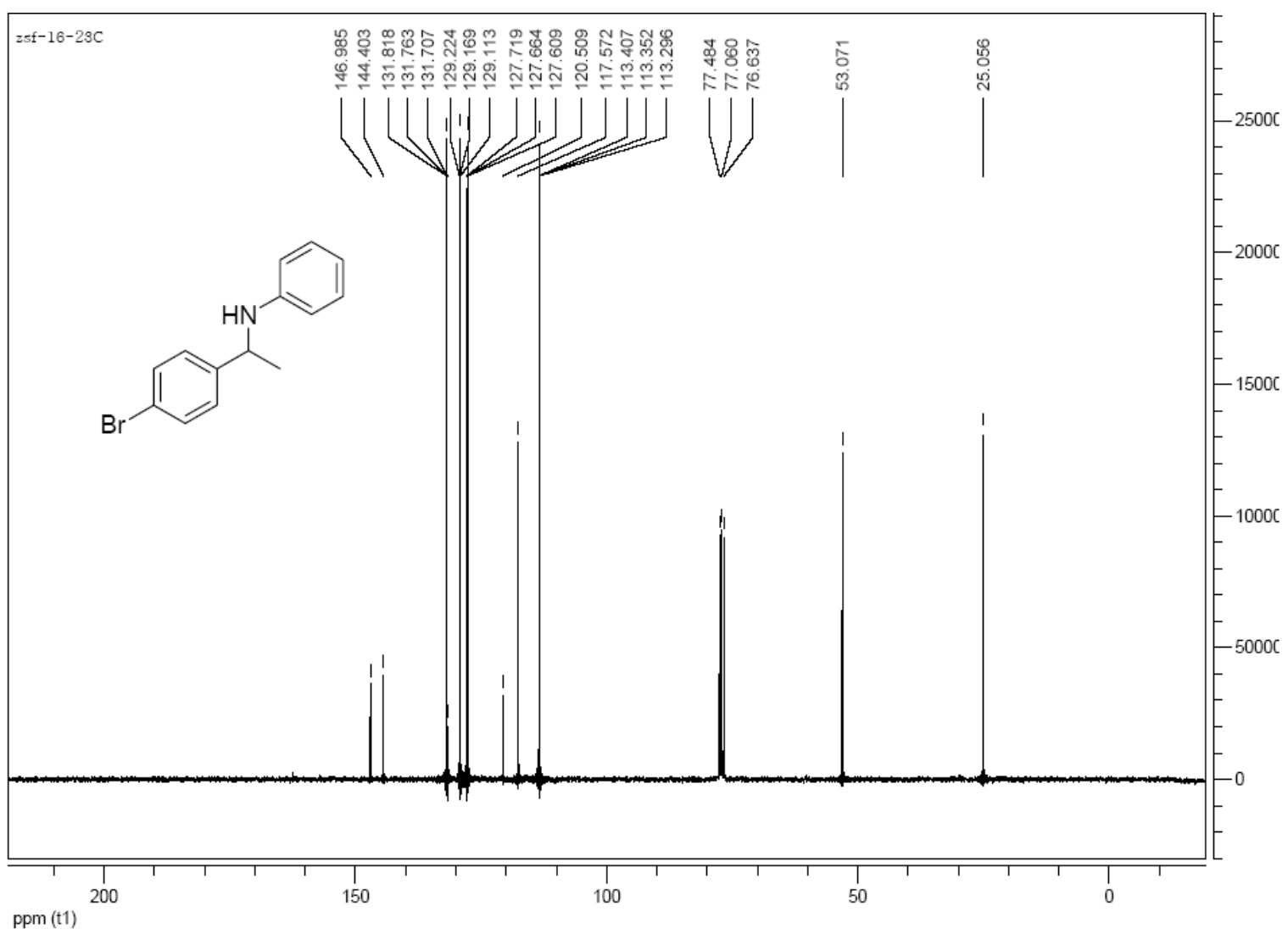



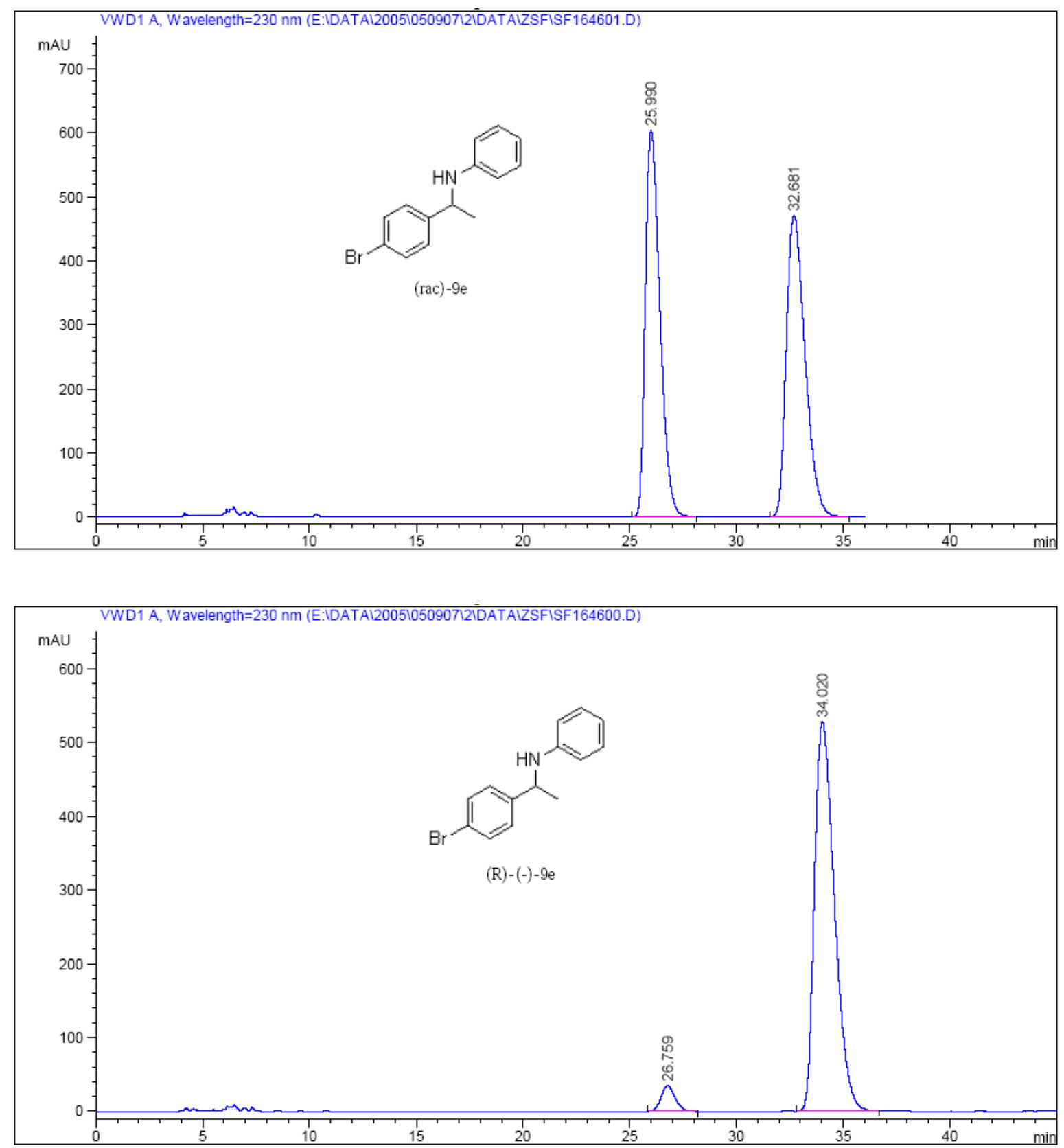

(-)-N-Phenyl-1-(3-chlorophenyl)ethylamine (9f): Colorless oil. $[\alpha]^{18}{ }_{D}-27\left(c 0.71, \mathrm{CH}_{2} \mathrm{Cl}_{2}\right)$; ${ }^{1} \mathrm{H}$ NMR (300 MHz, CDCl $\left.{ }_{3}\right) \delta 7.28$ (s, 1H, Ar-H), 7.16-6.99 (m, 5H, Ar-H), 6.58 (t, $J=7.2$ $\mathrm{Hz}, 1 \mathrm{H}, \mathrm{Ar}-\mathrm{H}$ ), 6.39 (d, $J=7.8 \mathrm{~Hz}, 2 \mathrm{H}, \mathrm{Ar}-\mathrm{H}$ ), 4.34 (dd, $J=6.6$ and $13.5 \mathrm{~Hz}, 1 \mathrm{H}, \mathrm{CH}$ ), 3.91 (bs, 1H, NH), 1.39 (d, $\left.J=6.9 \mathrm{~Hz}, 3 \mathrm{H}, \mathrm{CH}_{3}\right) ;{ }^{13} \mathrm{C} \mathrm{NMR}\left(75 \mathrm{MHz}, \mathrm{CDCl}_{3}\right) \delta$ 147.7, 147.0, 134.6, 130.0, 129.2, 127.1, 126.1, 124.1, 117.6, 113.4, 53.3, 25.1; HRMS (EI) calcd for $\mathrm{C}_{14} \mathrm{H}_{14} \mathrm{NCl}$ : 231.0815; Found 231.0816. Conversion was determined by GC using HP-5 column (programmed at $5{ }^{\circ} \mathrm{C} / \mathrm{min}$ from $100{ }^{\circ} \mathrm{C}$ to $220{ }^{\circ} \mathrm{C}$ ), $t_{\mathrm{R}}=17.84$ (product) and 18.25 (substrate) min. Enantiomer ratio was determined by HPLC using a Chiralcel OD-H column (flow rate $1.0 \mathrm{~mL} / \mathrm{min}, n-\mathrm{Hex} / \mathrm{iPrOH} 98: 2, \lambda=254 \mathrm{~nm}$ ), $t_{\mathrm{R}}=23.35 \mathrm{~min}(\operatorname{minor}), t_{\mathrm{R}}=32.65$ min (major); 93\% ee. 


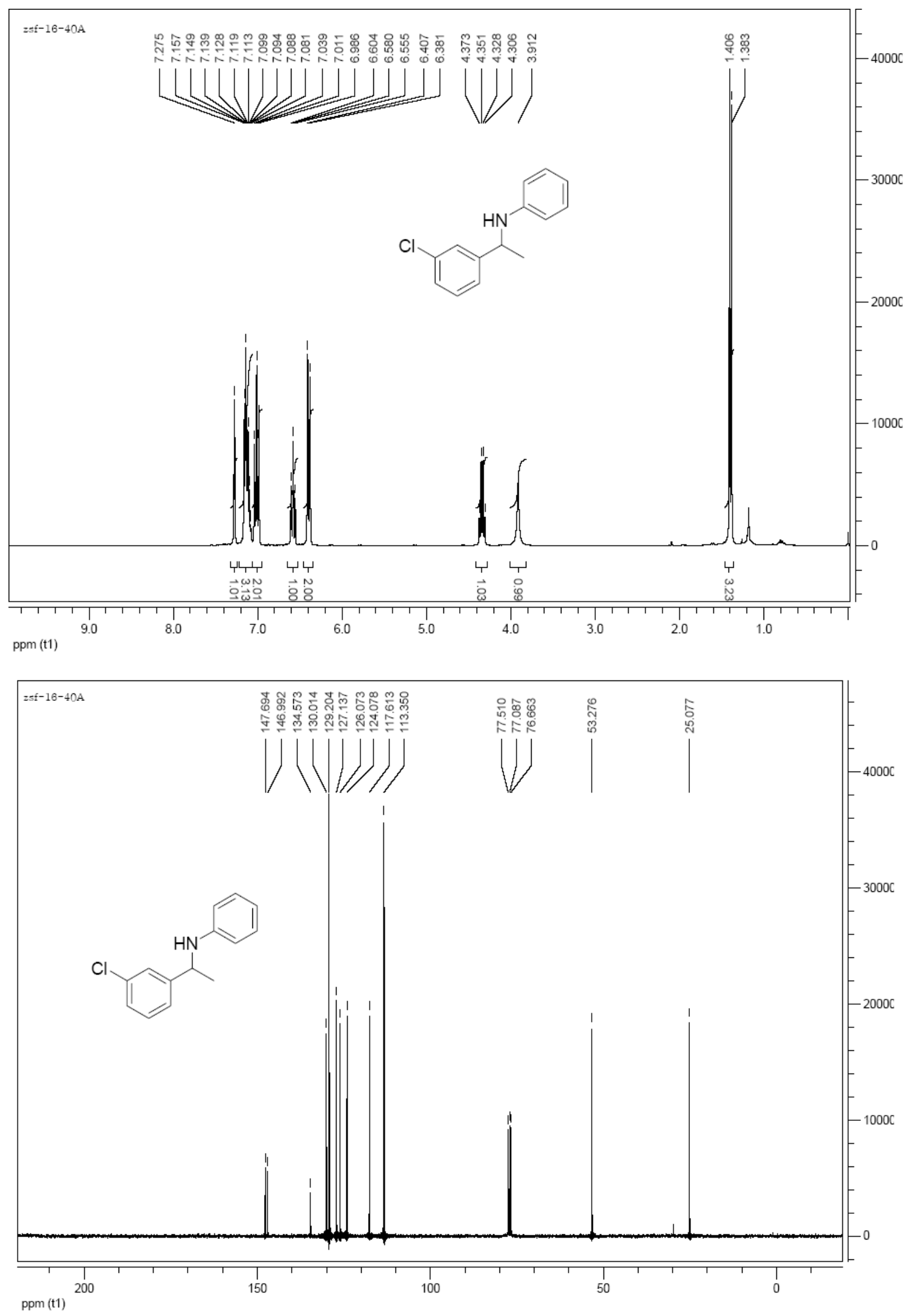



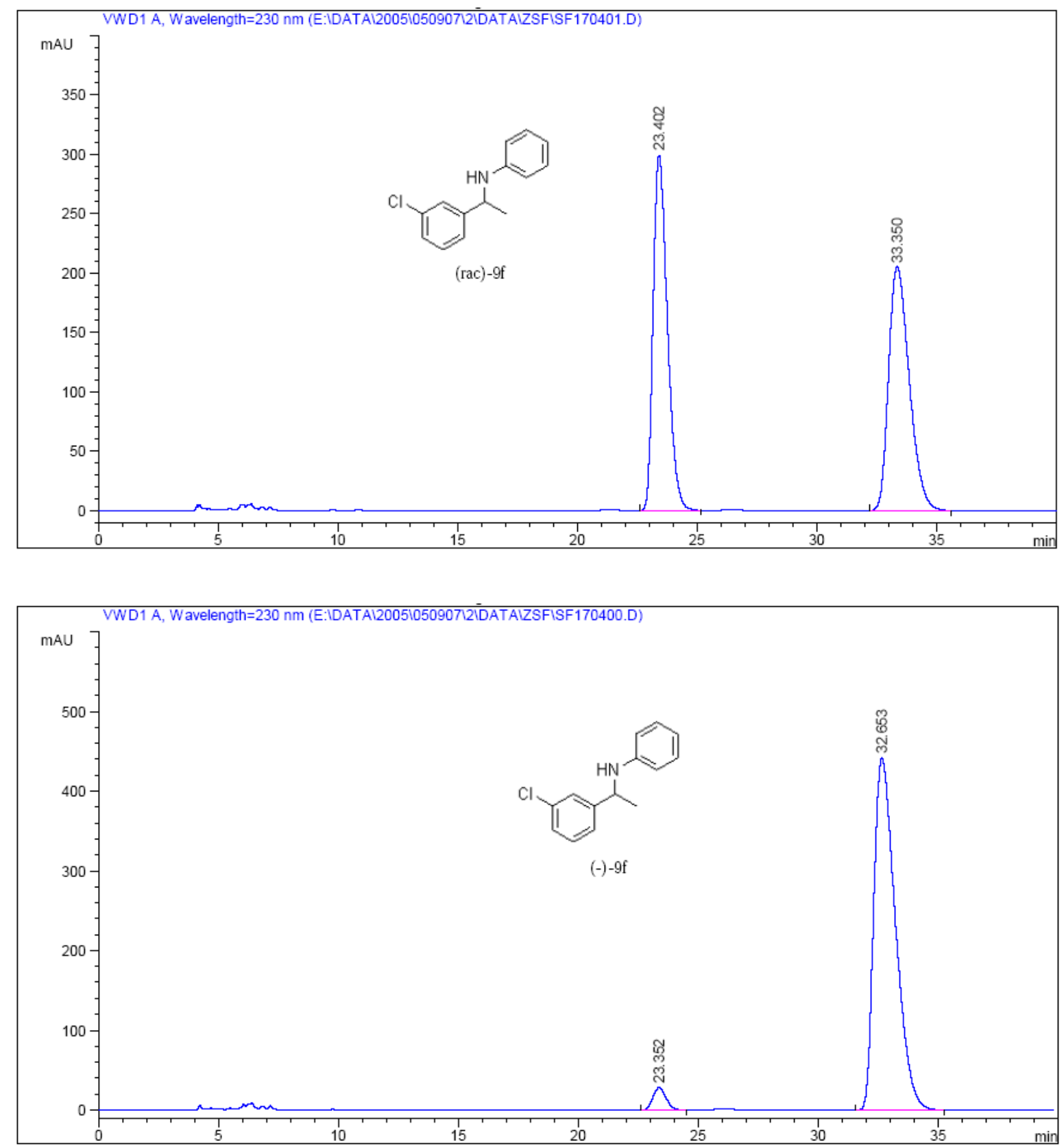

(-)- $\mathbf{N - P h e n y l - 1 - ( 3 - b r o m o p h e n y l ) e t h y l a m i n e ~ ( 9 g ) : ~ C o l o r l e s s ~ o i l . ~}[\alpha]_{\mathrm{D}}^{18}-18$ (c 0.865 , $\mathrm{CH}_{2} \mathrm{Cl}_{2}$ ); ${ }^{1} \mathrm{H}$ NMR (300 MHz, $\mathrm{CDCl}_{3}$ ) $\delta 7.43$ (s, 1H, Ar-H), 7.27-6.99 (m, 5H, Ar-H), 6.58 (t, $J=7.2 \mathrm{~Hz}, 1 \mathrm{H}, \mathrm{Ar}-\mathrm{H}$ ), 6.39 (d, $J=7.8 \mathrm{~Hz}, 2 \mathrm{H}, \mathrm{Ar}-\mathrm{H}$ ), 4.33 (dd, $J=6.6$ and $13.2 \mathrm{~Hz}, 1 \mathrm{H}, \mathrm{CH}$ ), 3.90 (bs, $1 \mathrm{H}, \mathrm{NH}), 1.39$ (d, $\left.J=6.9 \mathrm{~Hz}, 3 \mathrm{H}, \mathrm{CH}_{3}\right)$; ${ }^{13} \mathrm{C} \mathrm{NMR}\left(75 \mathrm{MHz}, \mathrm{CDCl}_{3}\right) \delta$ 148.0, 147.0, 130.3, 130.1, 129.2, 129.0, 124.5, 122.9, 117.6, 113.4, 53.3, 25.1; HRMS (EI) calcd for $\mathrm{C}_{14} \mathrm{H}_{14} \mathrm{NBr}$ : 275.0310; Found 275.0306. Conversion was determined by GC using HP-5 column (programmed at $5{ }^{\circ} \mathrm{C} / \mathrm{min}$ from $100{ }^{\circ} \mathrm{C}$ to $220{ }^{\circ} \mathrm{C}$ ), $t_{\mathrm{R}}=19.62$ (product) and 20.12 (substrate) min. Enantiomer ratio was determined by HPLC using a Chiralcel OD-H column (flow rate $1.0 \mathrm{~mL} / \mathrm{min}, n-\mathrm{Hex} / \mathrm{iPrOH} 98: 2, \lambda=254 \mathrm{~nm}$ ), $t_{\mathrm{R}}=24.66 \mathrm{~min}(\operatorname{minor}), t_{\mathrm{R}}=33.75$ min (major); 92\% ee. 


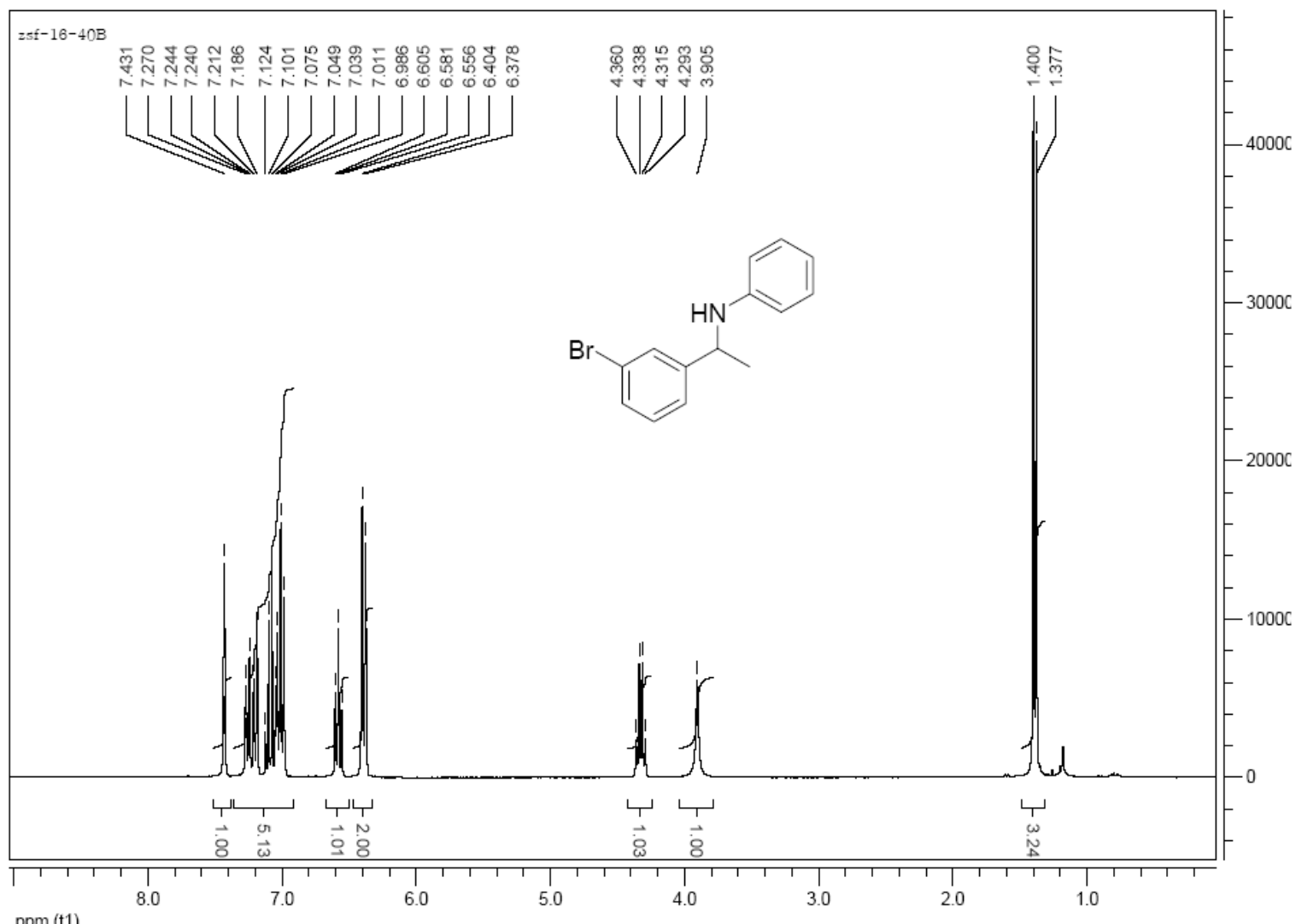

ppm (t1)

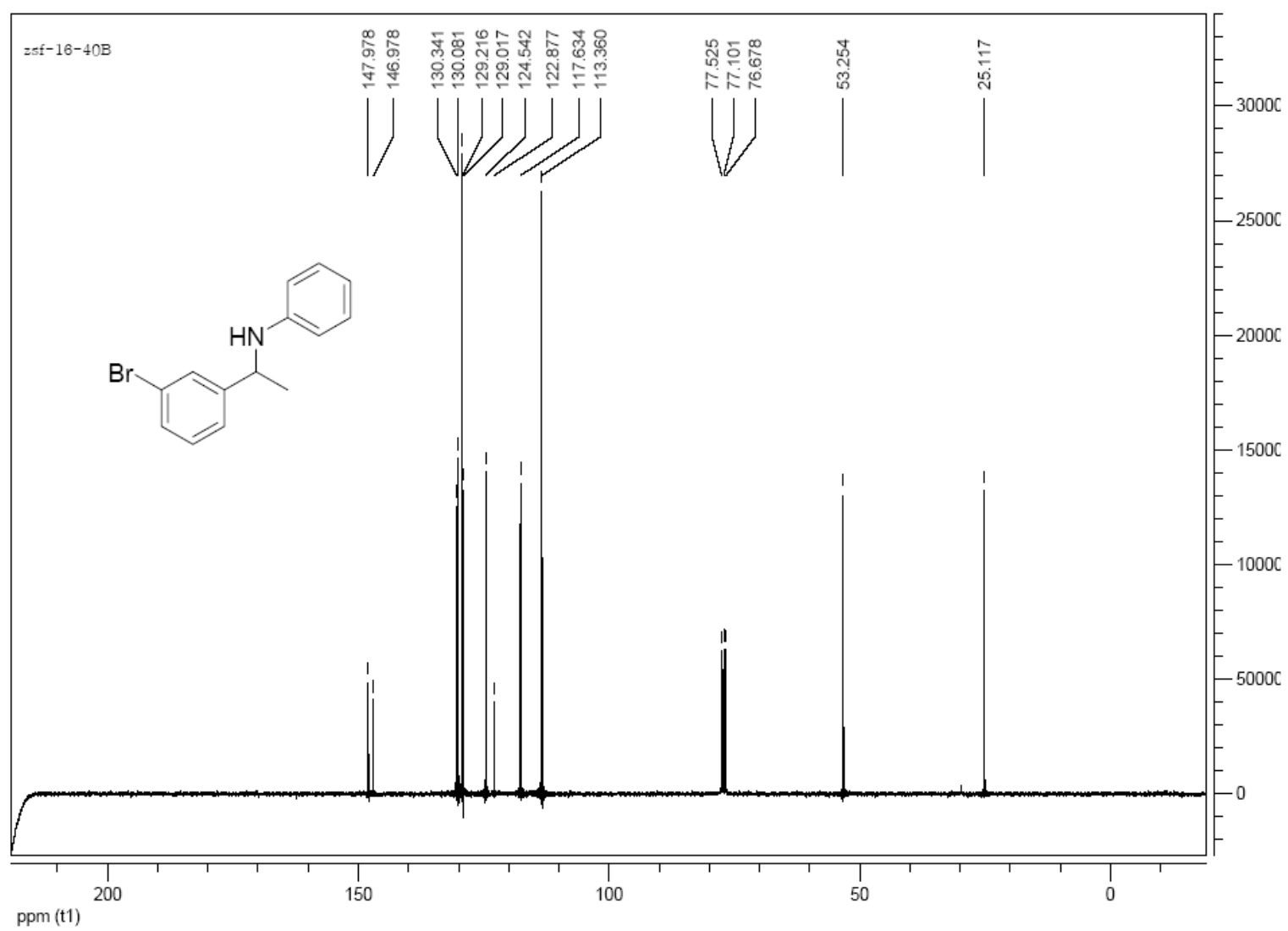



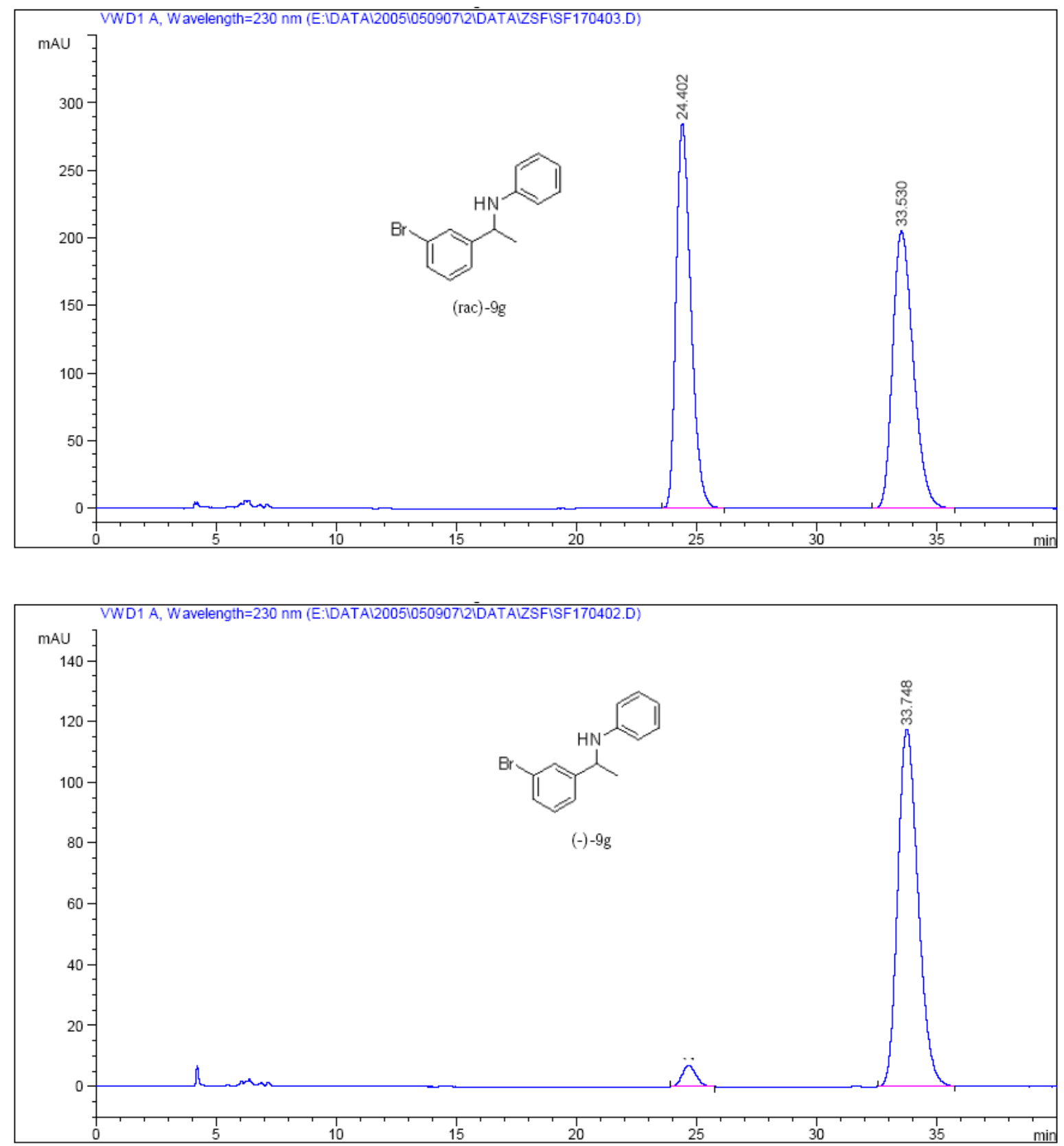

(-)- $\mathbf{N - P h e n y l - 1 - ( 3 , 4 - d i m e t h y l p h e n y l ) e t h y l a m i n e ~ ( 9 h ) : ~ C o l o r l e s s ~ o i l . ~}[\alpha]_{\mathrm{D}}^{18}-17$ (c 0.7 , $\mathrm{CH}_{2} \mathrm{Cl}_{2}$ ); ${ }^{1} \mathrm{H}$ NMR (300 MHz, $\left.\mathrm{CDCl}_{3}\right) \delta 7.10-6.98(\mathrm{~m}, 5 \mathrm{H}, \mathrm{Ar}-\mathrm{H}), 6.55$ (t, $J=7.5 \mathrm{~Hz}, 1 \mathrm{H}$, Ar-H), 6.43 (d, $J=7.8 \mathrm{~Hz}, 2 \mathrm{H}, \mathrm{Ar}-\mathrm{H}$ ), 4.33 (dd, $J=6.6$ and $13.2 \mathrm{~Hz}, 1 \mathrm{H}, \mathrm{CH}$ ), 3.90 (bs, 1H, $\mathrm{NH}$ ), 2.15 (s, 3H, CH3), 2.14 (s, 3H, CH3), 1.40 (d, $J=6.6 \mathrm{~Hz}, 3 \mathrm{H}, \mathrm{CH} 3) ;{ }^{13} \mathrm{C}$ NMR (75 $\left.\mathrm{MHz}, \mathrm{CDCl}_{3}\right) \delta 147.5,142.8,136.8,135.1,129.9,129.1,127.2,123.3,117.2,113.3,53.2$, 25.1, 20.0, 19.5; HRMS (EI) calcd for $\mathrm{C}_{16} \mathrm{H}_{19} \mathrm{~N}$ : 225.1517; Found 225.1513. Conversion was determined by GC using HP-5 column (programmed at $5{ }^{\circ} \mathrm{C} / \mathrm{min}$ from $100{ }^{\circ} \mathrm{C}$ to $220{ }^{\circ} \mathrm{C}$ ), $t_{\mathrm{R}}=$ 18.27 (product) and 19.42 (substrate) min. Enantiomer ratio was determined by HPLC using a Chiralcel OD-H column (flow rate $1.0 \mathrm{~mL} / \mathrm{min}, n-\mathrm{Hex} / \mathrm{iPrOH} 98: 2, \lambda=254 \mathrm{~nm}$ ), $t_{\mathrm{R}}=11.36$ $\min (\operatorname{minor}), t_{\mathrm{R}}=12.87 \mathrm{~min}$ (major); $94 \%$ ee. 


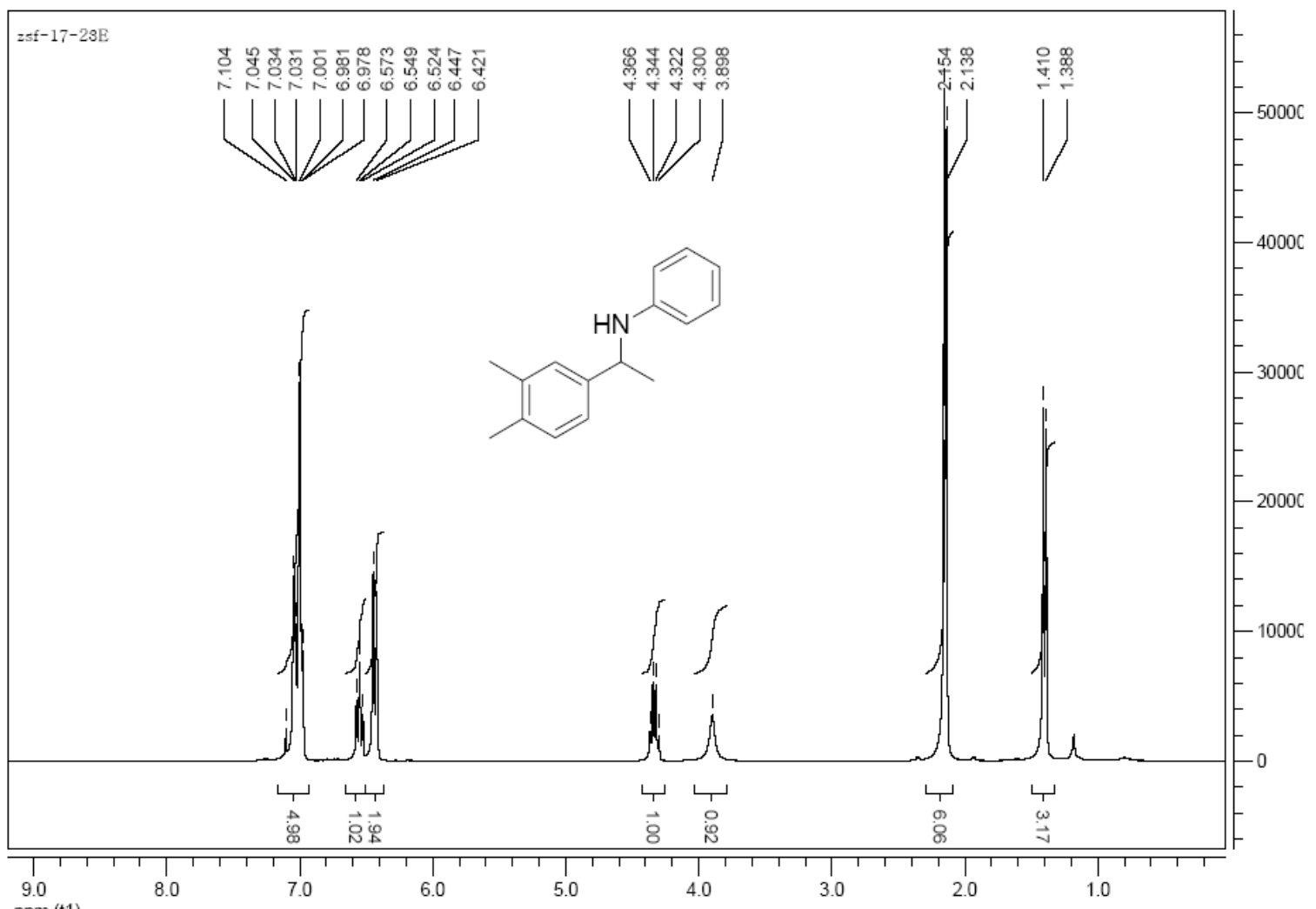

$\mathrm{ppm}$ (t1)

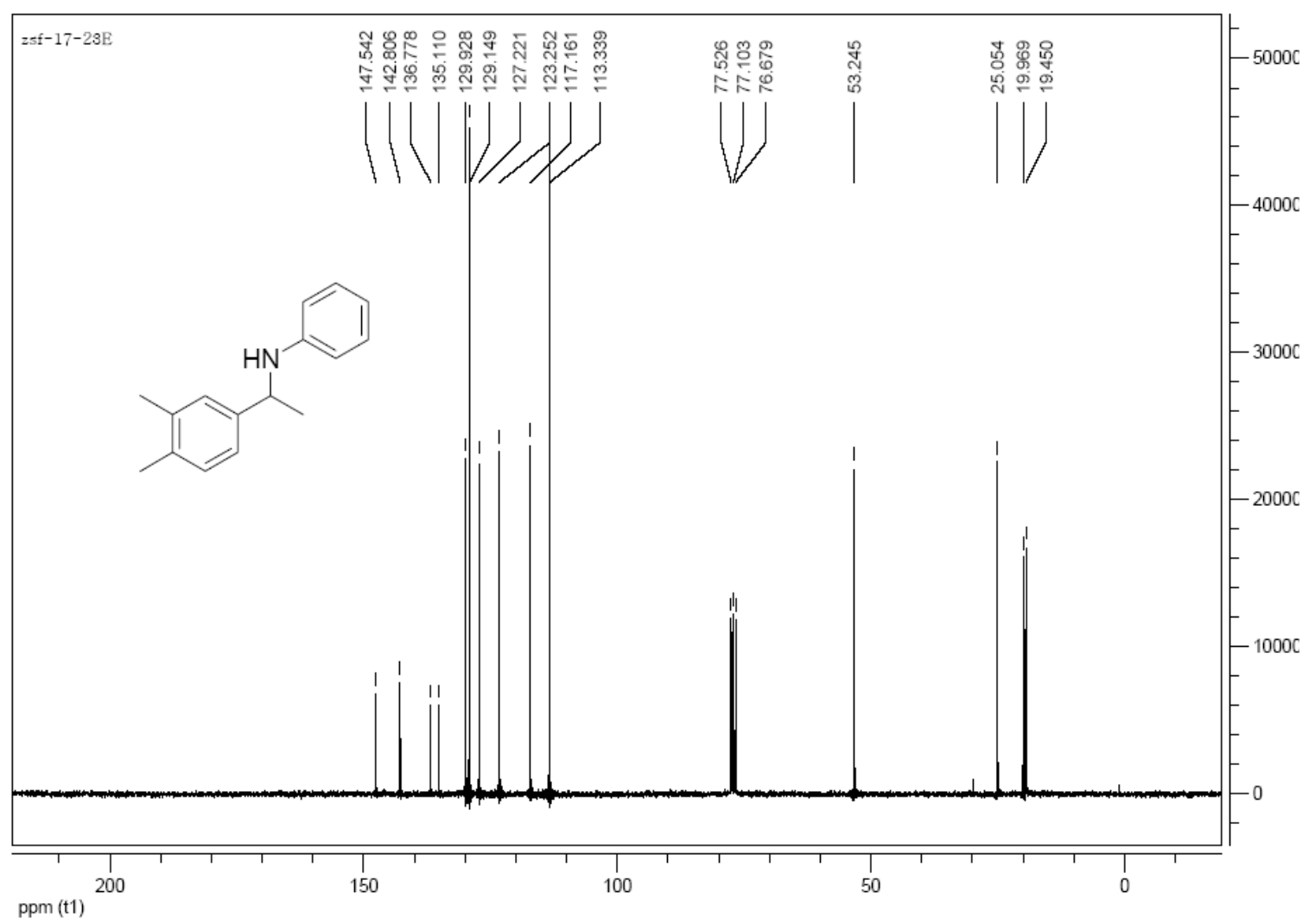



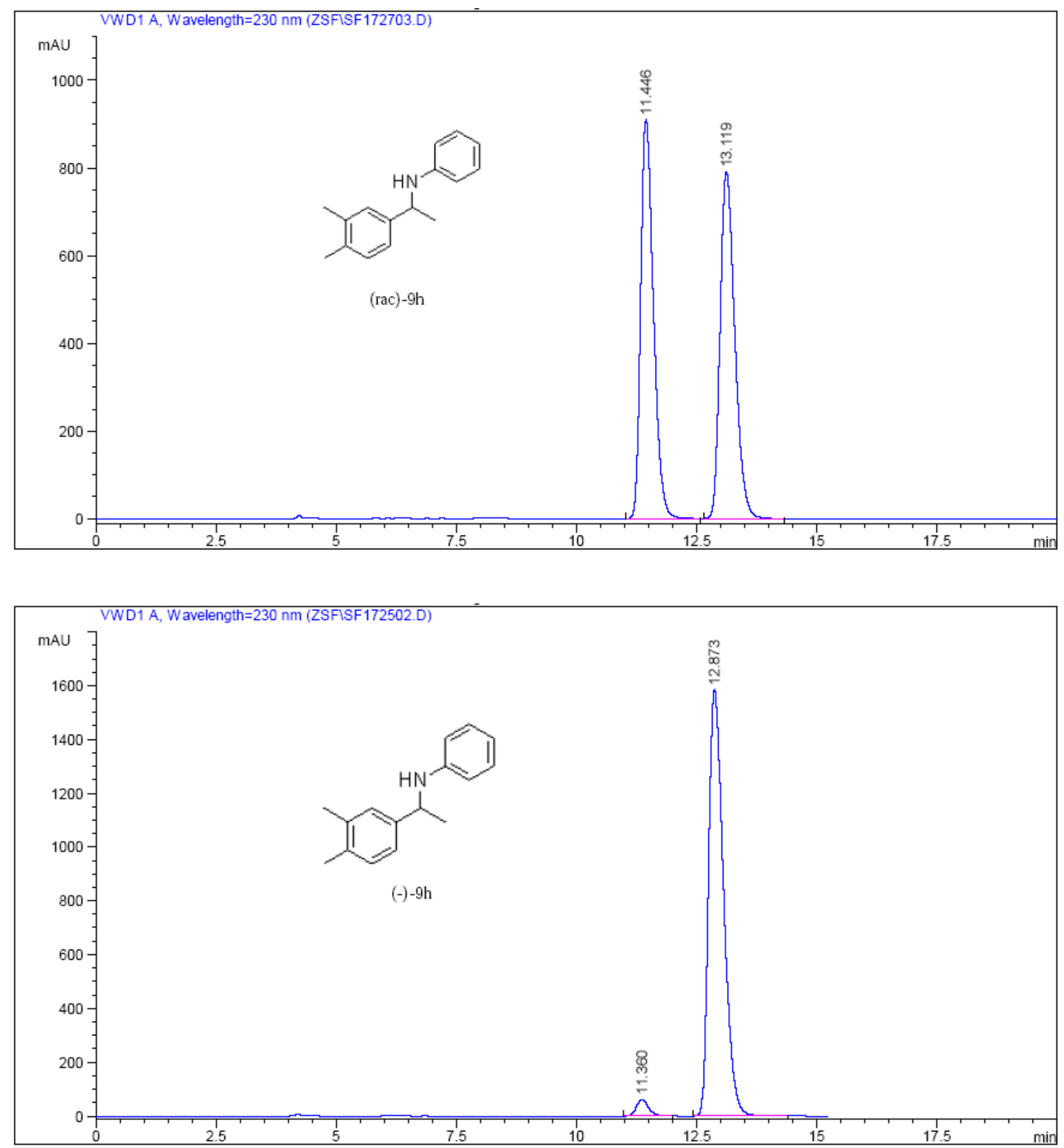

(-)- $N$-(4-Methylphenyl)-1-phenylethylamine (9i): ${ }^{7}$ White solid. Mp $55-58{ }^{\circ} \mathrm{C}$; $[\alpha]^{18}{ }_{\mathrm{D}}-25(\mathrm{C}$ 1.345, $\mathrm{CH}_{2} \mathrm{Cl}_{2}$ ); ${ }^{1} \mathrm{H}$ NMR (300 MHz, $\left.\mathrm{CDCl}_{3}\right) \delta$ 7.30-7.11 (m, 5H, Ar-H), 6.82 (d, $J=8.1 \mathrm{~Hz}$, 2H, Ar-H), 6.35 (d, $J=8.4 \mathrm{~Hz}, 2 \mathrm{H}, \mathrm{Ar}-\mathrm{H}$ ), 4.37 (dd, $J=6.6$ and $13.2 \mathrm{~Hz}, 1 \mathrm{H}, \mathrm{CH}$ ), 3.82 (bs, $1 \mathrm{H}, \mathrm{NH}), 2.10\left(\mathrm{~s}, 3 \mathrm{H}, \mathrm{CH}_{3}\right), 1.42\left(\mathrm{~d}, J=6.6 \mathrm{~Hz}, 3 \mathrm{H}, \mathrm{CH}_{3}\right)$. Conversion was determined by GC using HP-5 column (programmed at $5{ }^{\circ} \mathrm{C} / \mathrm{min}$ from $100{ }^{\circ} \mathrm{C}$ to $220{ }^{\circ} \mathrm{C}$ ), $t_{\mathrm{R}}=16.03$ (product) and 16.77 (substrate) min. Enantiomer ratio was determined by HPLC using a Chiralcel OD-H column (flow rate $1.0 \mathrm{~mL} / \mathrm{min}, n-\mathrm{Hex} / \mathrm{iPrOH} 98: 2, \lambda=254 \mathrm{~nm}$ ), $t_{\mathrm{R}}=14.12$ $\min$ (major), $t_{\mathrm{R}}=16.90 \mathrm{~min}$ (minor); $93 \%$ ee. 

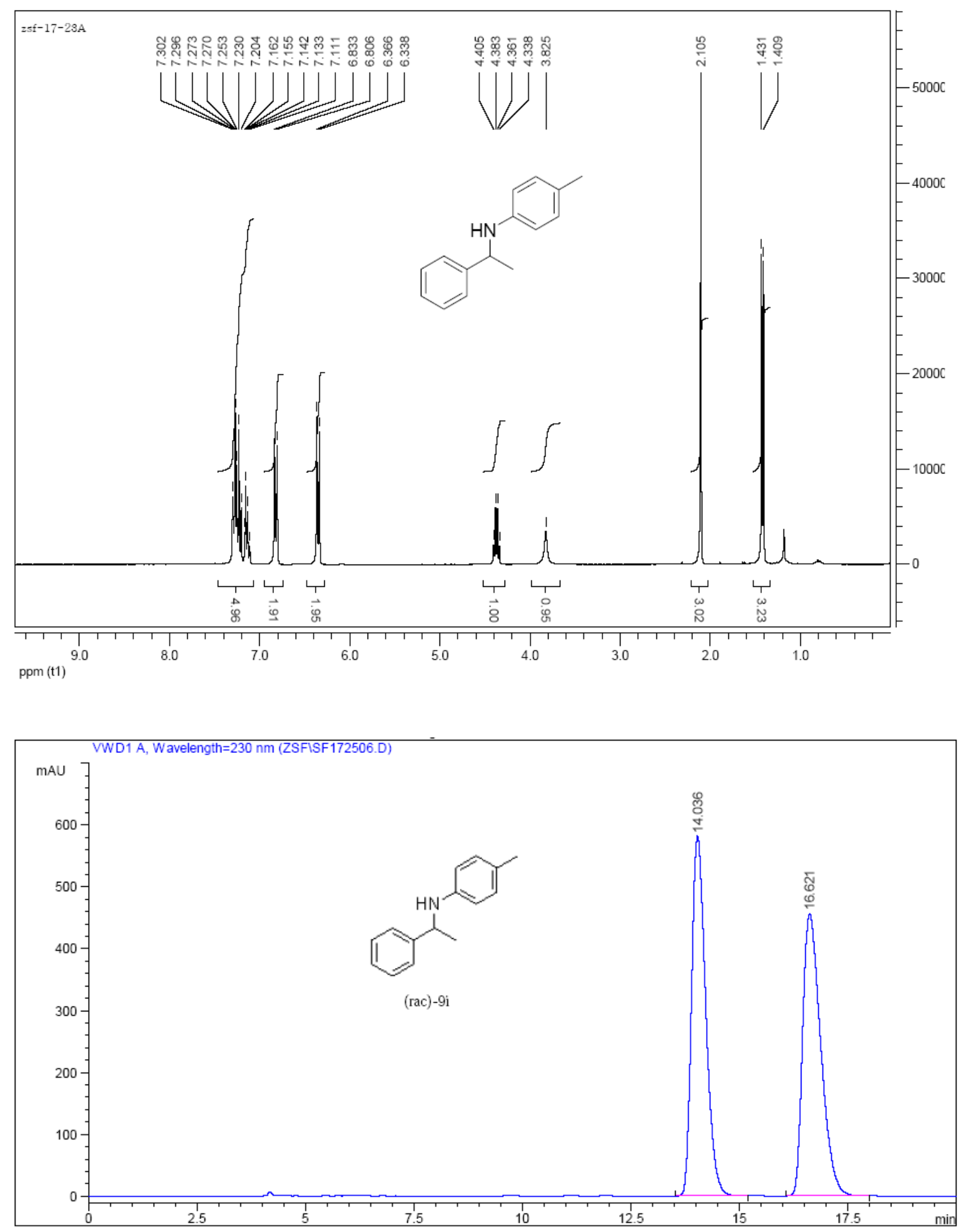


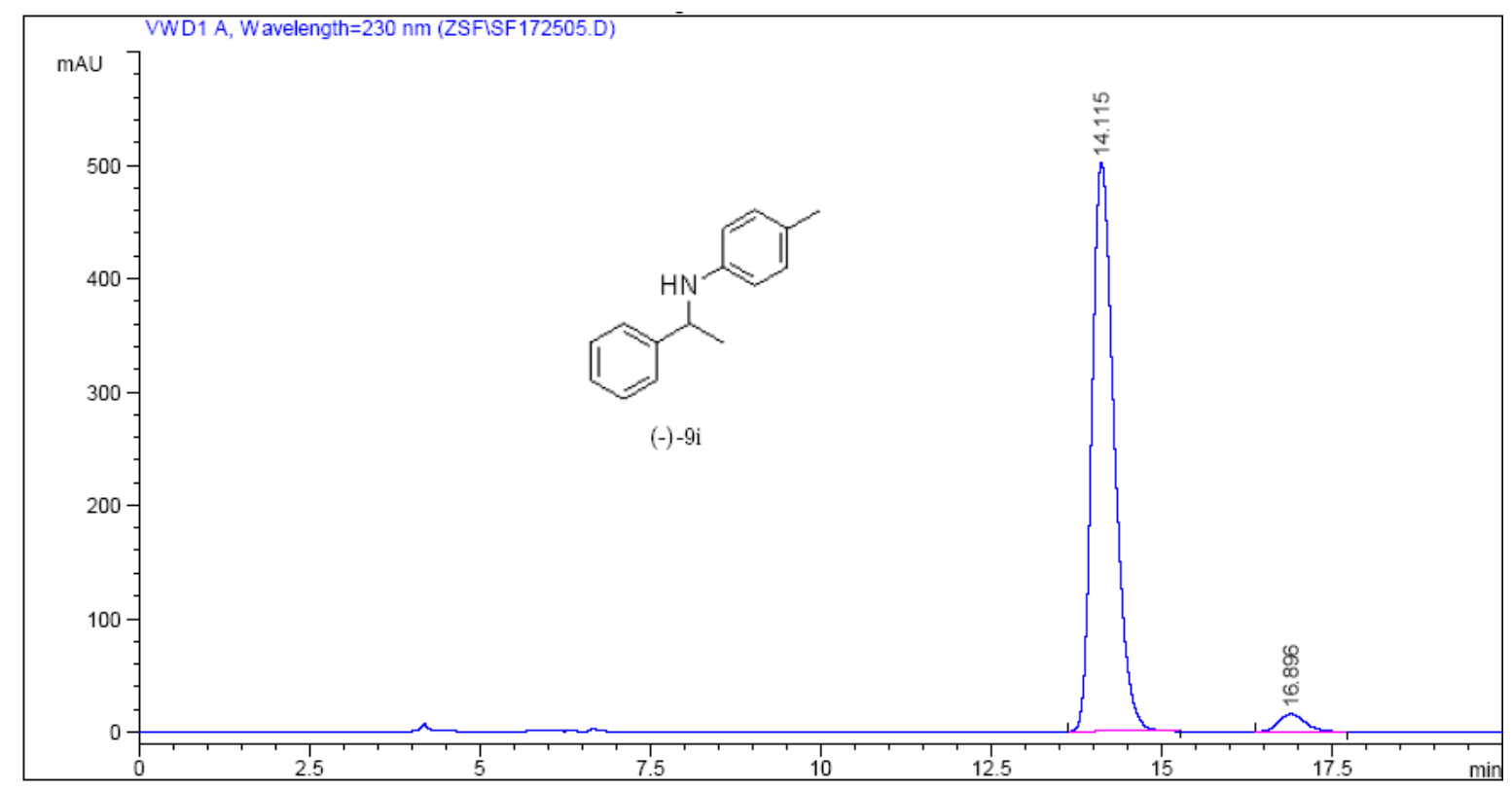

(-)- $N$-(4-Chlorophenyl)-1-phenylethylamine (9j): ${ }^{8}$ Colorless oil. $[\alpha]^{18}{ }_{D}-8$ (c 1.13, $\left.\mathrm{CH}_{2} \mathrm{Cl}_{2}\right)$; ${ }^{1} \mathrm{H}$ NMR (300 MHz, CDCl $\left.{ }_{3}\right) \delta$ 7.33-7.20 (m, 5H, Ar-H), 7.04-7.00 (m, 2H, Ar-H), 6.44-6.38 (m, 2H, Ar-H), 4.43 (dd, $J=6.6$ and $13.5 \mathrm{~Hz}, 1 \mathrm{H}, \mathrm{CH}$ ), 4.05 (bs, $1 \mathrm{H}, \mathrm{NH}$ ), 1.50 (d, $J=6.6 \mathrm{~Hz}$, $3 \mathrm{H}, \mathrm{CH}_{3}$ ). Conversion was determined by ${ }^{1} \mathrm{H}$ NMR. Enantiomer ratio was determined by HPLC using a Chiralcel OD-H column (flow rate $1.0 \mathrm{~mL} / \mathrm{min}, n-\mathrm{Hex} / \mathrm{iPrOH} 98: 2, \lambda=254$ $\mathrm{nm}), t_{\mathrm{R}}=18.07 \min$ (major), $t_{\mathrm{R}}=27.36 \mathrm{~min}$ (minor); 97\% ee.

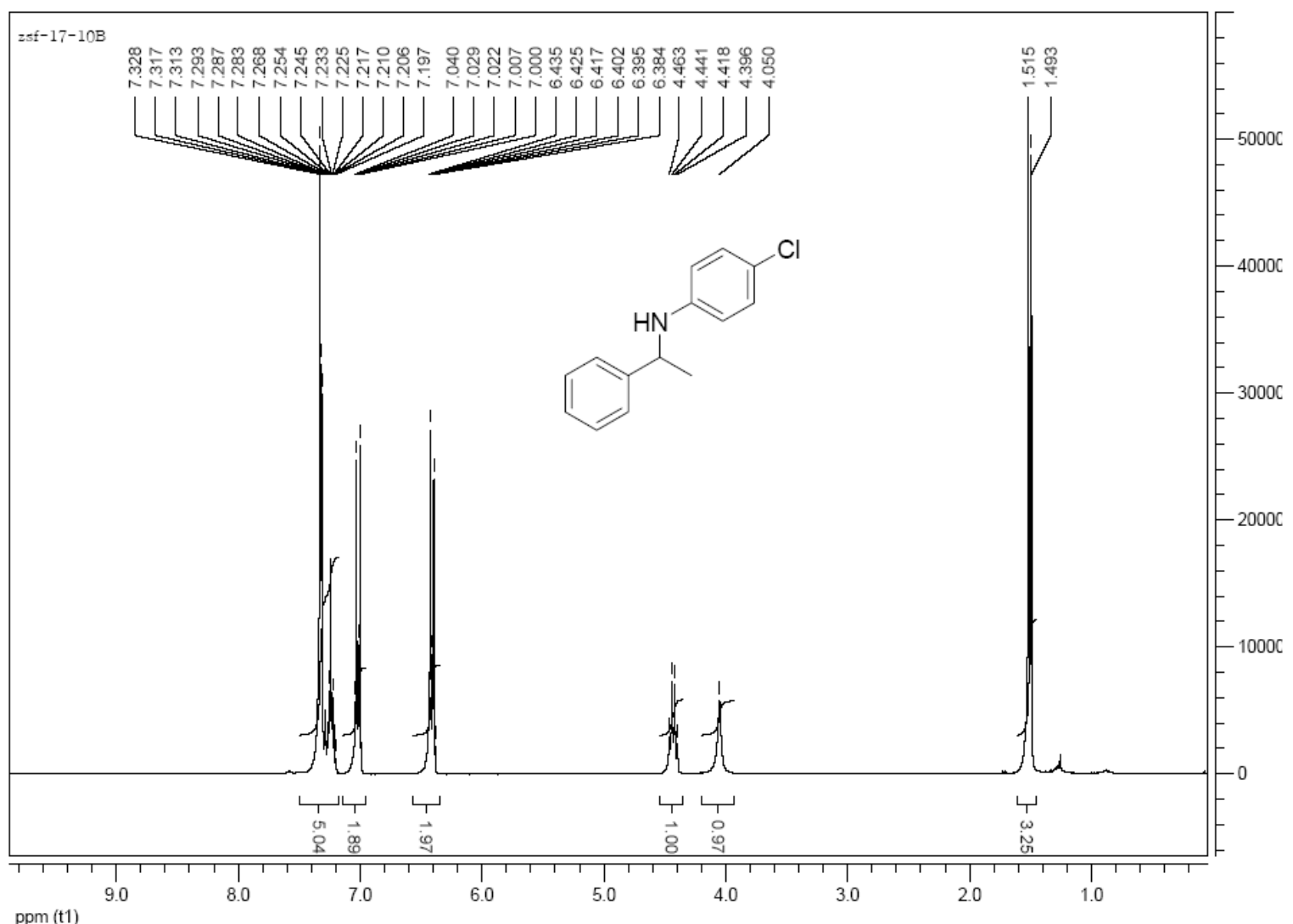



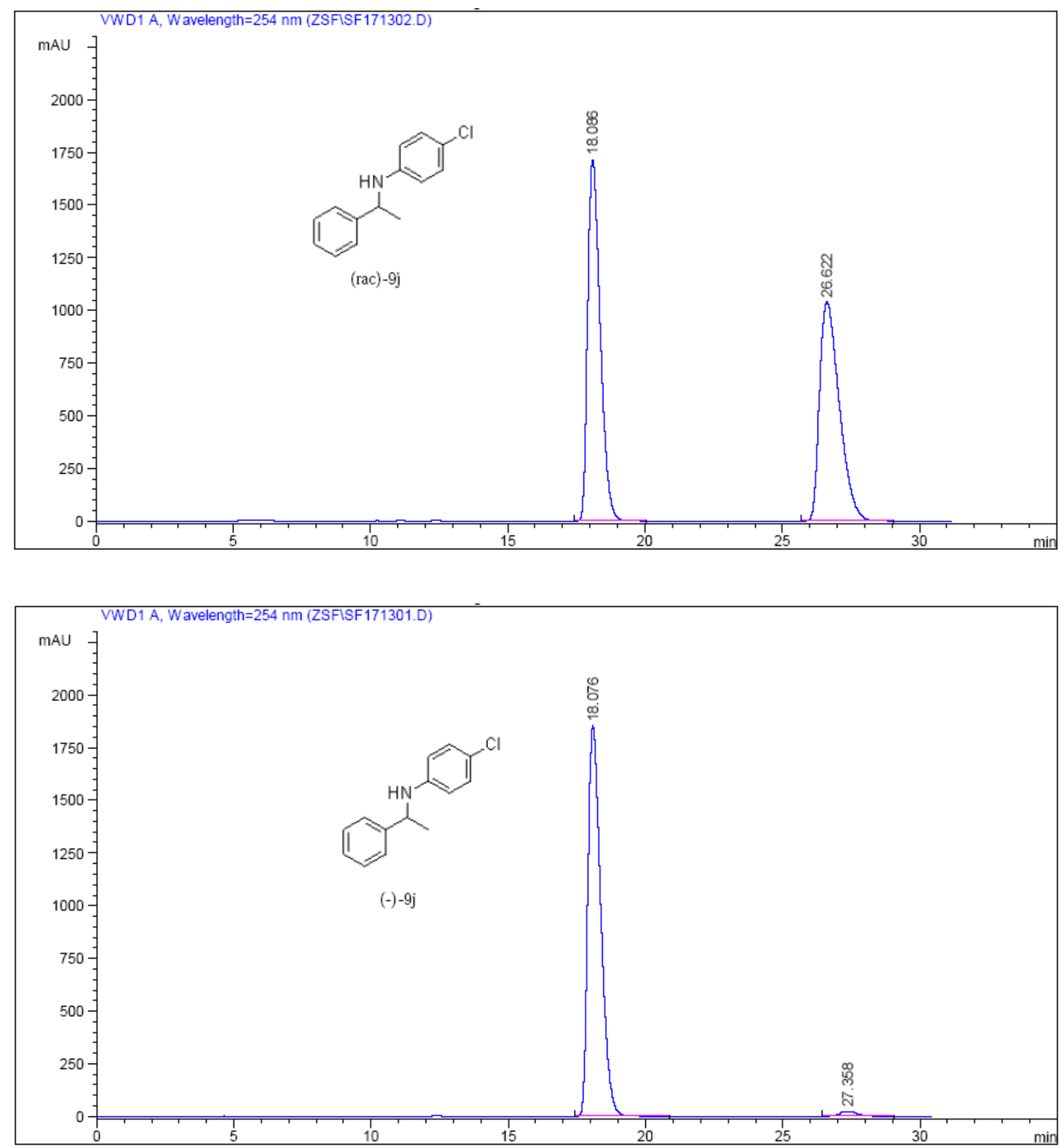

(+)- $\mathbf{N - ( 4 - B r o m o p h e n y l ) - 1 - p h e n y l e t h y l a m i n e ~ ( 9 k ) : ~ C o l o r l e s s ~ o i l . ~}[\alpha]_{\mathrm{D}}^{18}+4 \quad$ (c 0.985 , $\mathrm{CH}_{2} \mathrm{Cl}_{2}$ ); ${ }^{1} \mathrm{H}$ NMR (300 MHz, $\left.\mathrm{CDCl}_{3}\right) \delta$ 7.24-7.03 (m, 7H, Ar-H), 6.30-6.25 (m, 2H, Ar-H), 4.33 (dd, $J=6.6$ and $13.2 \mathrm{~Hz}, 1 \mathrm{H}, \mathrm{CH}$ ), 3.97 (bs, $1 \mathrm{H}, \mathrm{NH}$ ), 1.41 (d, $\left.J=6.6 \mathrm{~Hz}, 3 \mathrm{H}, \mathrm{CH}_{3}\right) ;{ }^{13} \mathrm{C}$ NMR $\left(75 \mathrm{MHz}, \mathrm{CDCl}_{3}\right) \delta 146.2,144.7,131.8,128.8,127.1,125.8,114.9,108.9,53.5,25.0$; HRMS (EI) calcd for $\mathrm{C}_{14} \mathrm{H}_{14} \mathrm{NBr}$ : 275.0310; Found 275.0304. Conversion was determined by ${ }^{1} \mathrm{H}$ NMR. Enantiomer ratio was determined by HPLC using a Chiralcel OD-H column (flow rate $1.0 \mathrm{~mL} / \mathrm{min}, n$-Hex/iPrOH 98:2, $\lambda=254 \mathrm{~nm}$ ), $t_{\mathrm{R}}=16.57 \mathrm{~min}$ (major), $t_{\mathrm{R}}=23.56 \mathrm{~min}$ (minor); 96\% ee. 


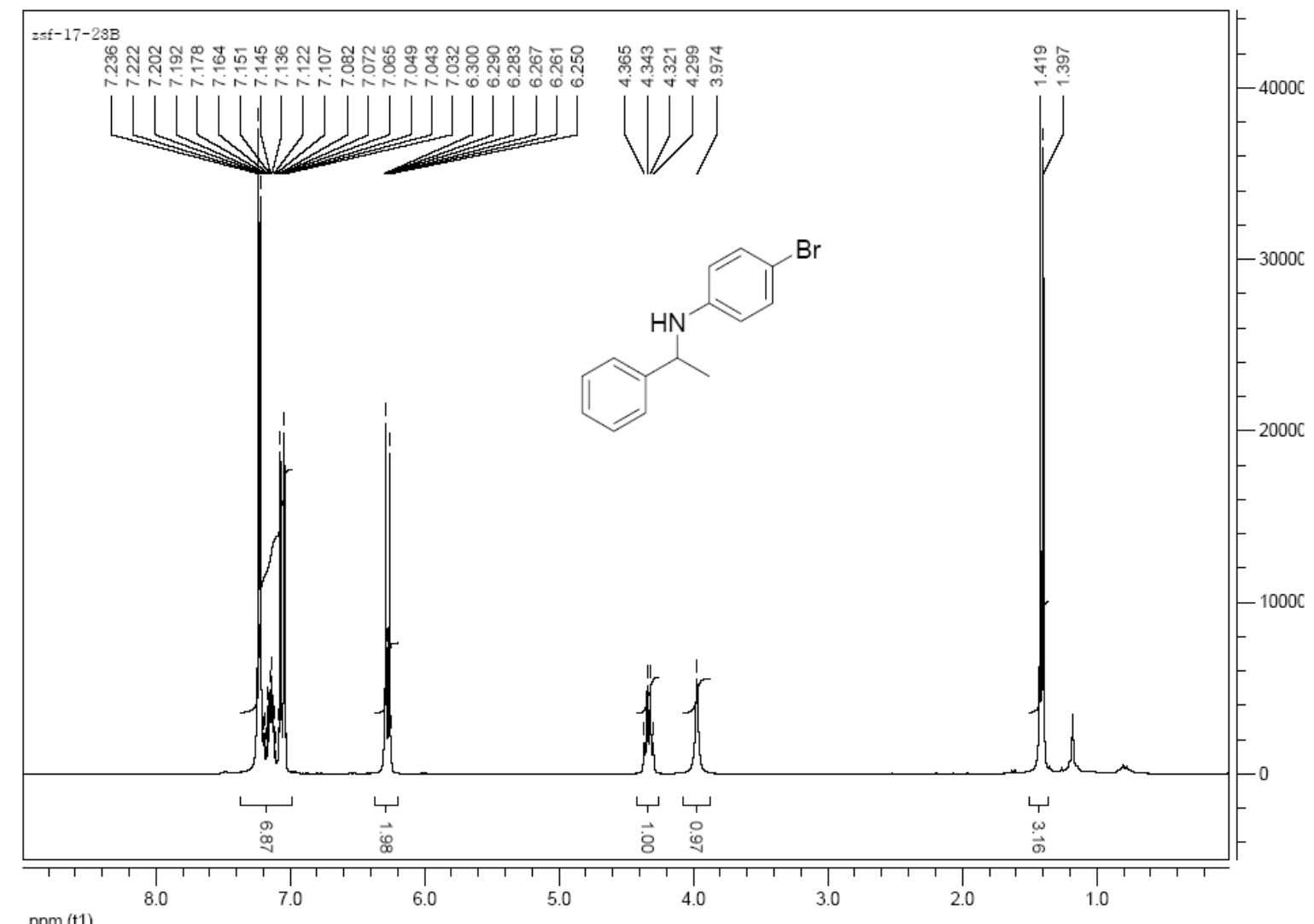

ppm (t1)

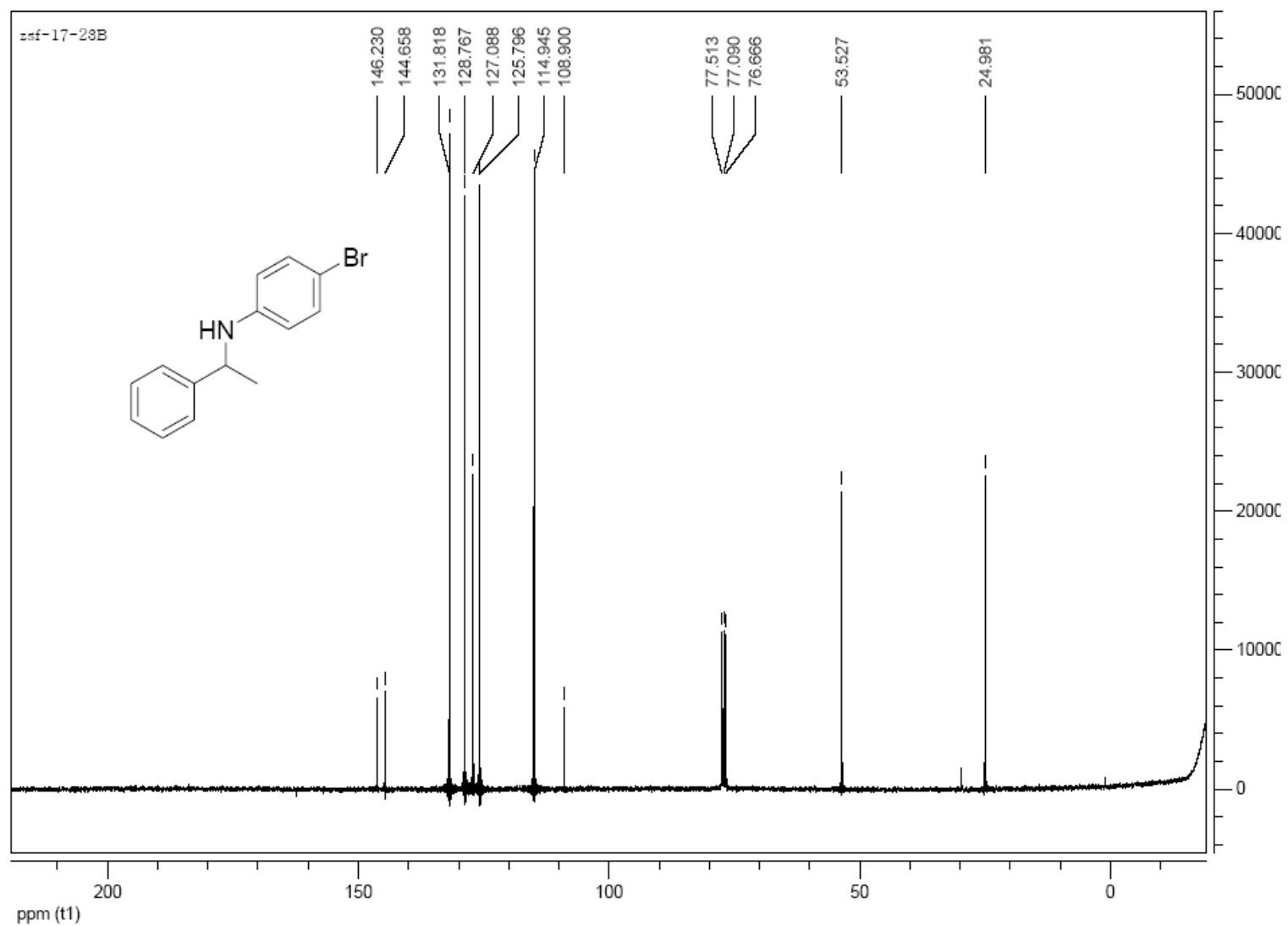



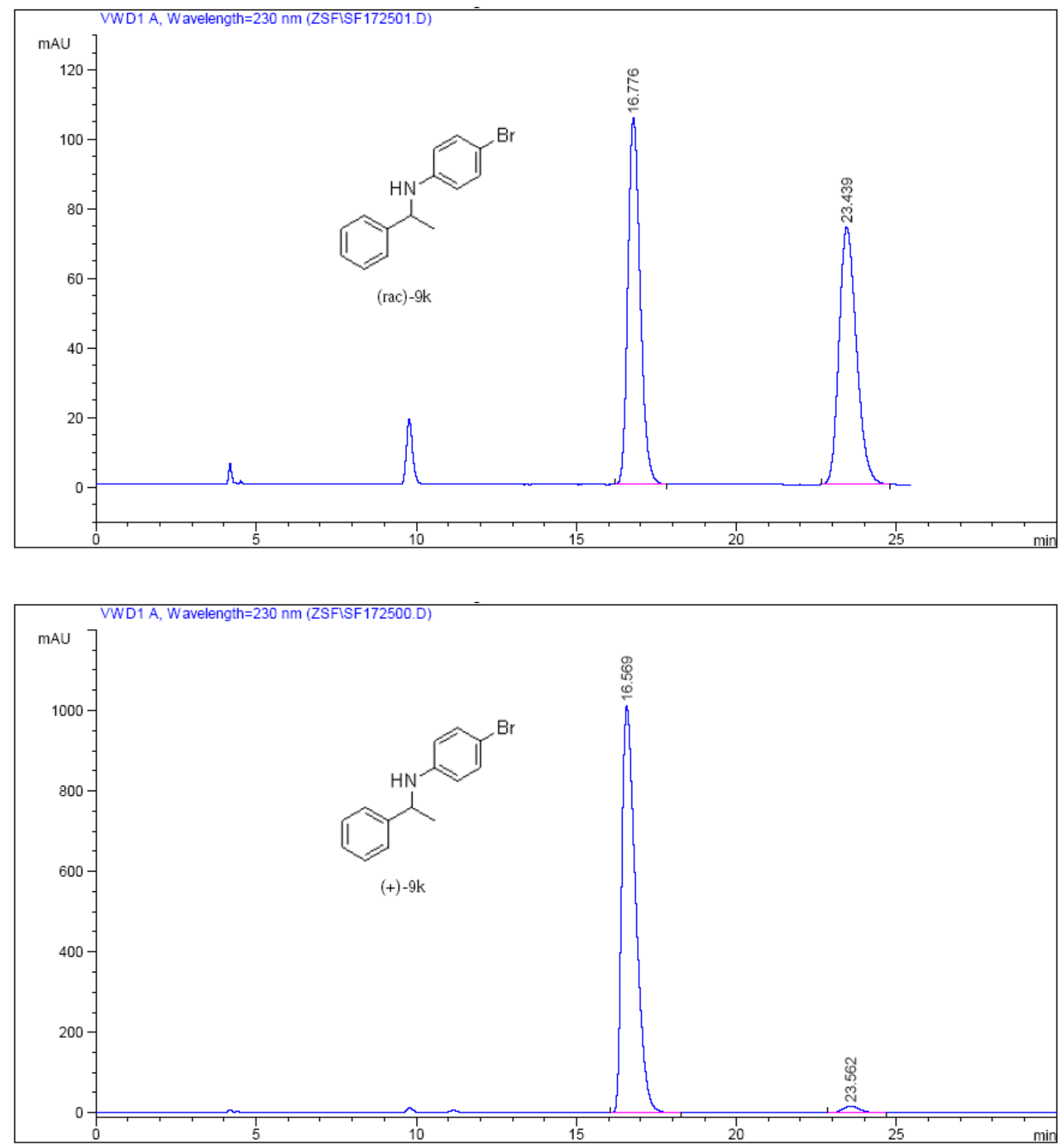

(-)- $N$-(3-Methylphenyl)-1-phenylethylamine (9l): ${ }^{9}$ Colorless oil. $[\alpha]^{18}-31$ (c 0.685 , $\mathrm{CH}_{2} \mathrm{Cl}_{2}$ ); ${ }^{1} \mathrm{H}$ NMR (300 MHz, $\left.\mathrm{CDCl}_{3}\right) \delta 7.38-7.20(\mathrm{~m}, 5 \mathrm{H}, \mathrm{Ar}-\mathrm{H}), 6.97$ (t, $J=7.5 \mathrm{~Hz}, 1 \mathrm{H}$, Ar-H), 6.48-6.29 (m, 3H, Ar-H), 4.48 (dd, $J=6.6$ and $13.2 \mathrm{~Hz}, 1 \mathrm{H}, \mathrm{CH}), 3.97$ (bs, $1 \mathrm{H}, \mathrm{NH}$ ), 2.21 (s, 3H, $\left.\mathrm{CH}_{3}\right), 1.50\left(\mathrm{~d}, \mathrm{~J}=6.6 \mathrm{~Hz}, 3 \mathrm{H}, \mathrm{CH}_{3}\right.$ ). Conversion was determined by GC using HP-5 column (programmed at $5{ }^{\circ} \mathrm{C} / \mathrm{min}$ from $100{ }^{\circ} \mathrm{C}$ to $220{ }^{\circ} \mathrm{C}$ ), $t_{\mathrm{R}}=15.71$ (product) and 16.45 (substrate) min. Enantiomer ratio was determined by HPLC using a Chiralcel OD-H column (flow rate $1.0 \mathrm{~mL} / \mathrm{min}, n-\mathrm{Hex} / \mathrm{iPrOH} 98: 2, \lambda=254 \mathrm{~nm}$ ), $t_{\mathrm{R}}=12.01 \mathrm{~min}$ (minor), $t_{\mathrm{R}}=$ $16.57 \mathrm{~min}$ (major); $91 \%$ ee. 

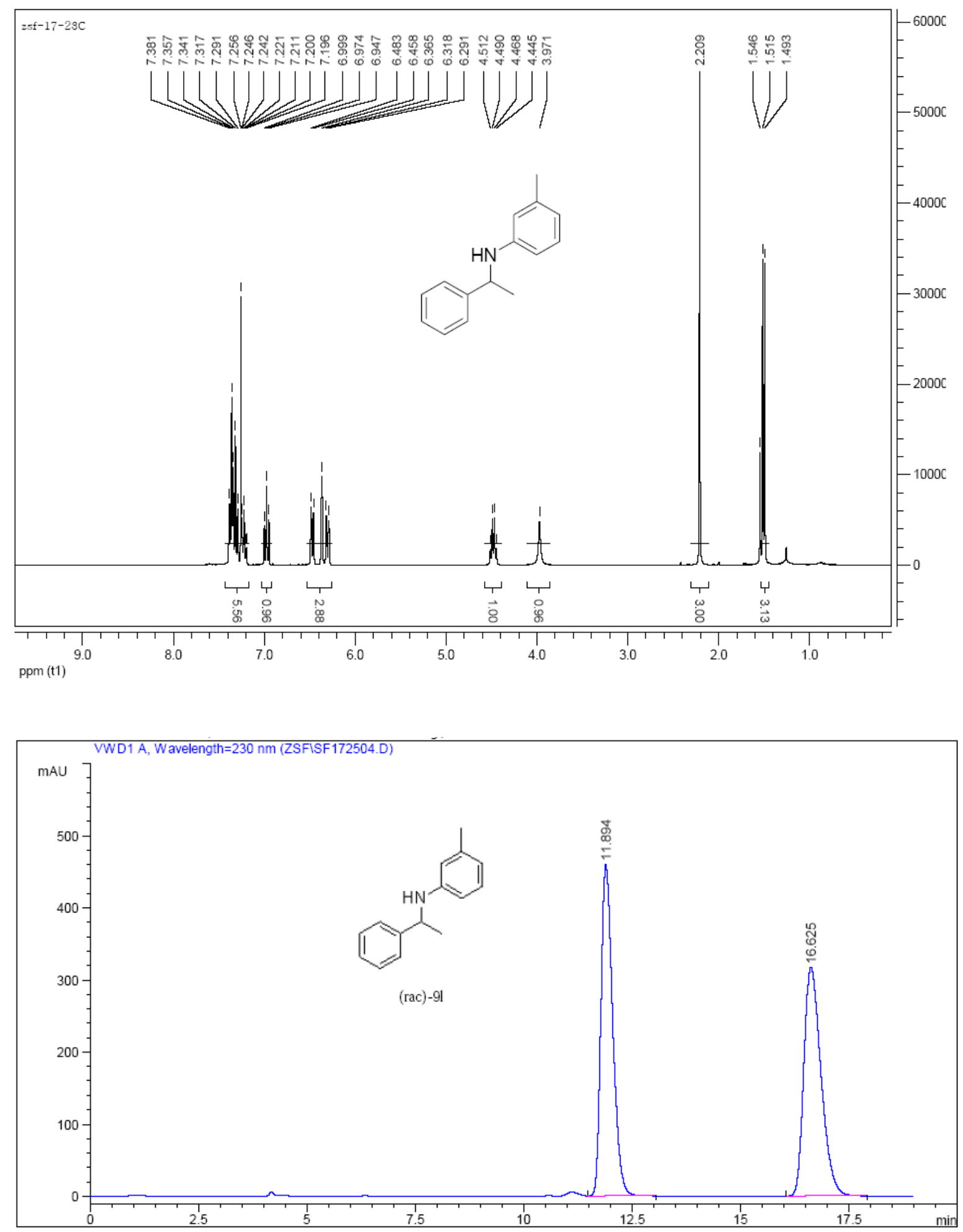


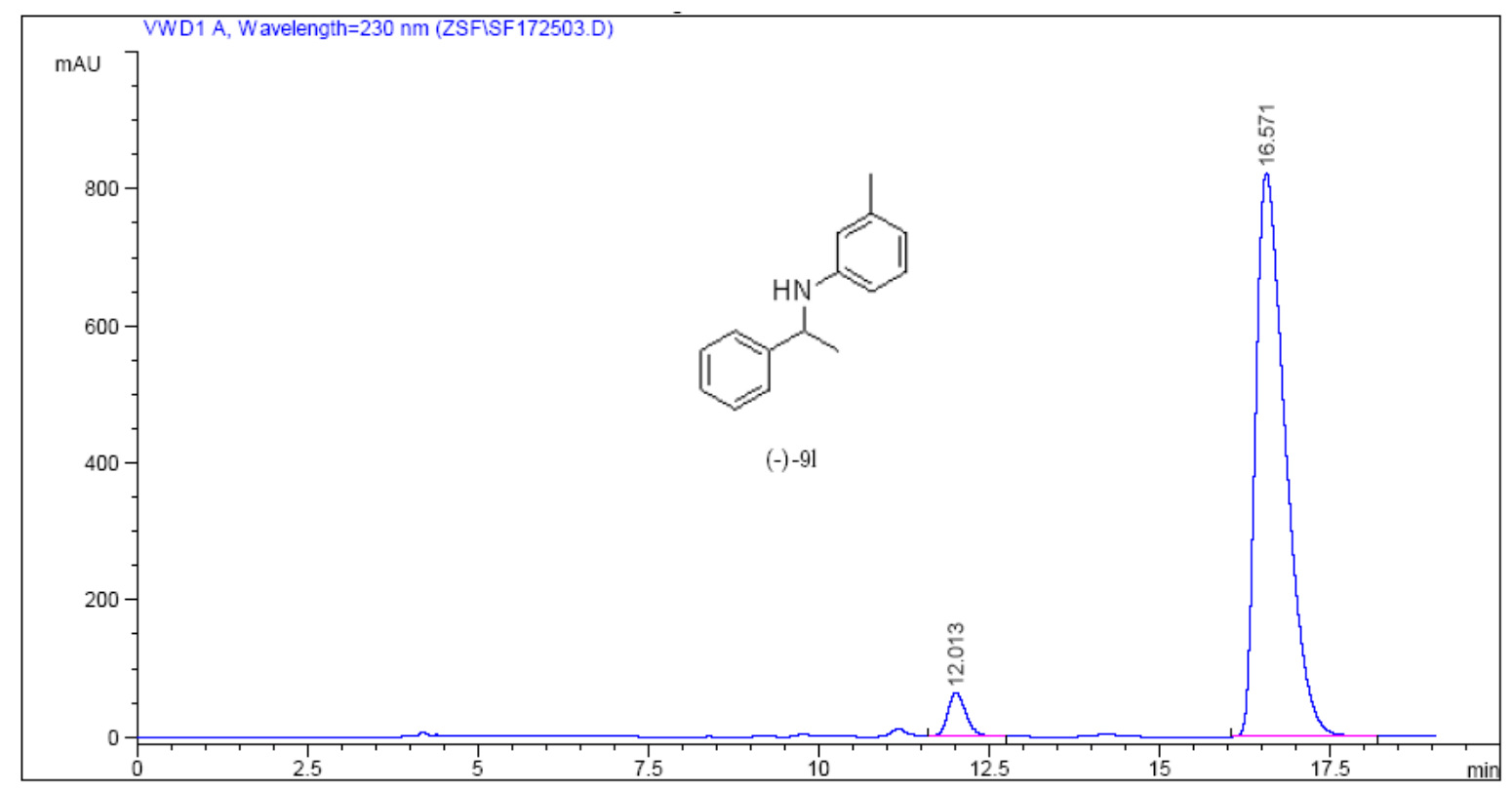

(-)- $N$-(3-Bromophenyl)-1-phenylethylamine (9m): Colorless oil. $[\alpha]_{D}^{18}-13$ (c 0.97 , $\mathrm{CH}_{2} \mathrm{Cl}_{2}$ ); ${ }^{1} \mathrm{H}$ NMR (300 MHz, $\left.\mathrm{CDCl}_{3}\right) \delta 7.32-7.19(\mathrm{~m}, 5 \mathrm{H}, \mathrm{Ar}-\mathrm{H}), 6.90$ (t, $J=8.1 \mathrm{~Hz}, 1 \mathrm{H}$, Ar-H), 6.73 (d, $J=7.2 \mathrm{~Hz}, 1 \mathrm{H}, \mathrm{Ar}-\mathrm{H}), 6.65$ (t, $J=2.1 \mathrm{~Hz}, 1 \mathrm{H}, \mathrm{Ar}-\mathrm{H}), 6.37$ (dd, $J=1.5$ and $8.1 \mathrm{~Hz}, 1 \mathrm{H}, \mathrm{Ar}-\mathrm{H}$ ), 4.43 (dd, $J=6.6$ and $13.2 \mathrm{~Hz}, 1 \mathrm{H}, \mathrm{CH}$ ), 4.07 (bs, 1H, NH), 1.48 (d, $J=$ $\left.6.9 \mathrm{~Hz}, 3 \mathrm{H}, \mathrm{CH}_{3}\right) ;{ }^{13} \mathrm{C} \mathrm{NMR}\left(75 \mathrm{MHz}, \mathrm{CDCl}_{3}\right) \delta$ 148.6, 144.5, 130.4, 128.8, 127.1, 125.8, 123.1, 120.1, 116.1, 111.9, 53.3, 24.8; HRMS (EI) calcd for $\mathrm{C}_{14} \mathrm{H}_{14} \mathrm{NBr}$ : 275.0310; Found 275.0304. Conversion was determined by GC using HP-5 column (programmed at $5{ }^{\circ} \mathrm{C} / \mathrm{min}$ from $100{ }^{\circ} \mathrm{C}$ to $220{ }^{\circ} \mathrm{C}$ ), $t_{\mathrm{R}}=19.92$ (product) and 20.21 (substrate) min. Enantiomer ratio was determined by HPLC using a Chiralcel OD-H column (flow rate $1.0 \mathrm{~mL} / \mathrm{min}, n-\mathrm{Hex} / \mathrm{iPrOH}$ 98:2, $\lambda=254 \mathrm{~nm}$ ), $t_{\mathrm{R}}=19.60 \min \left(\right.$ minor), $t_{\mathrm{R}}=26.80 \min$ (major); $94 \%$ ee. 


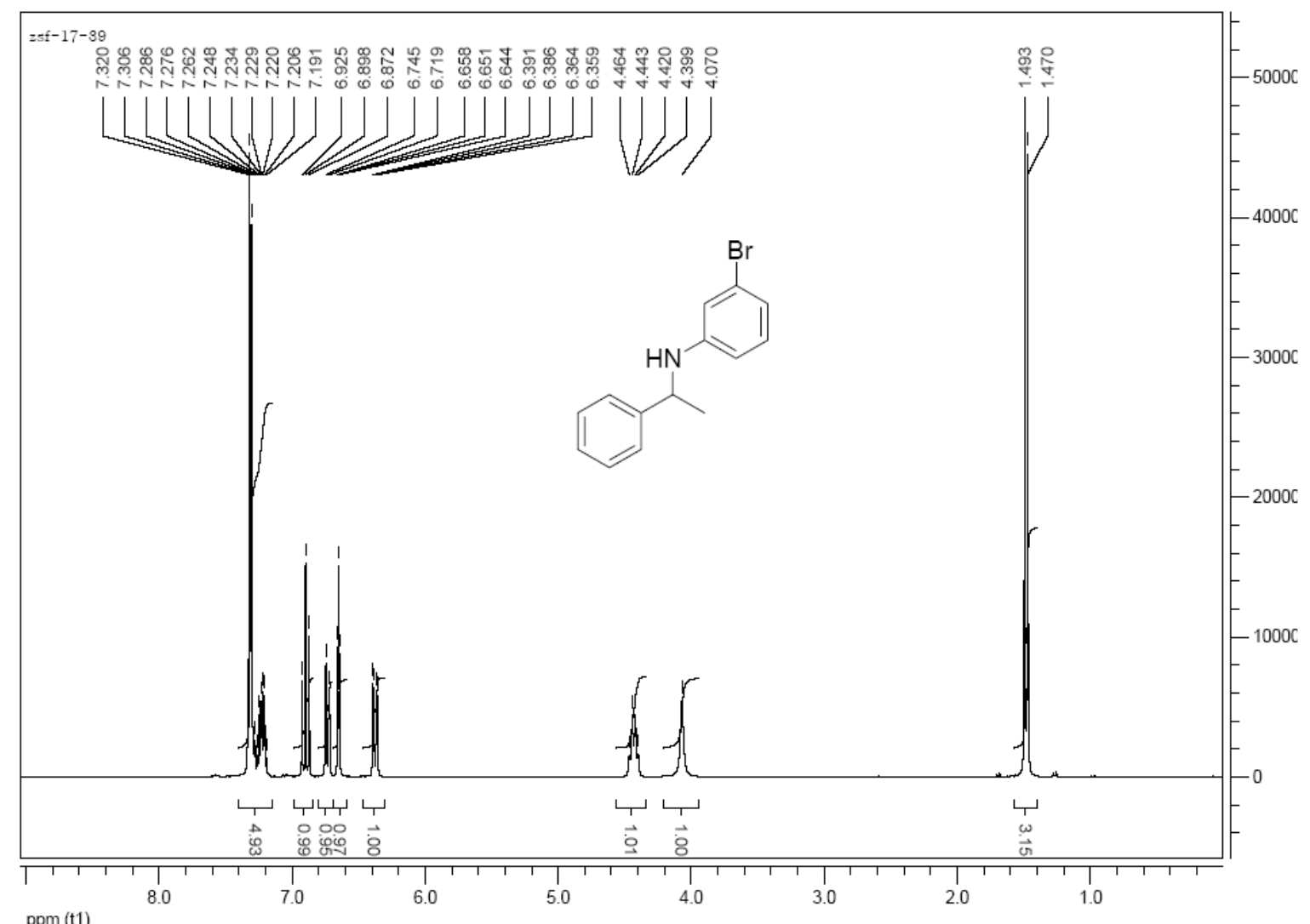

ppm (t1)

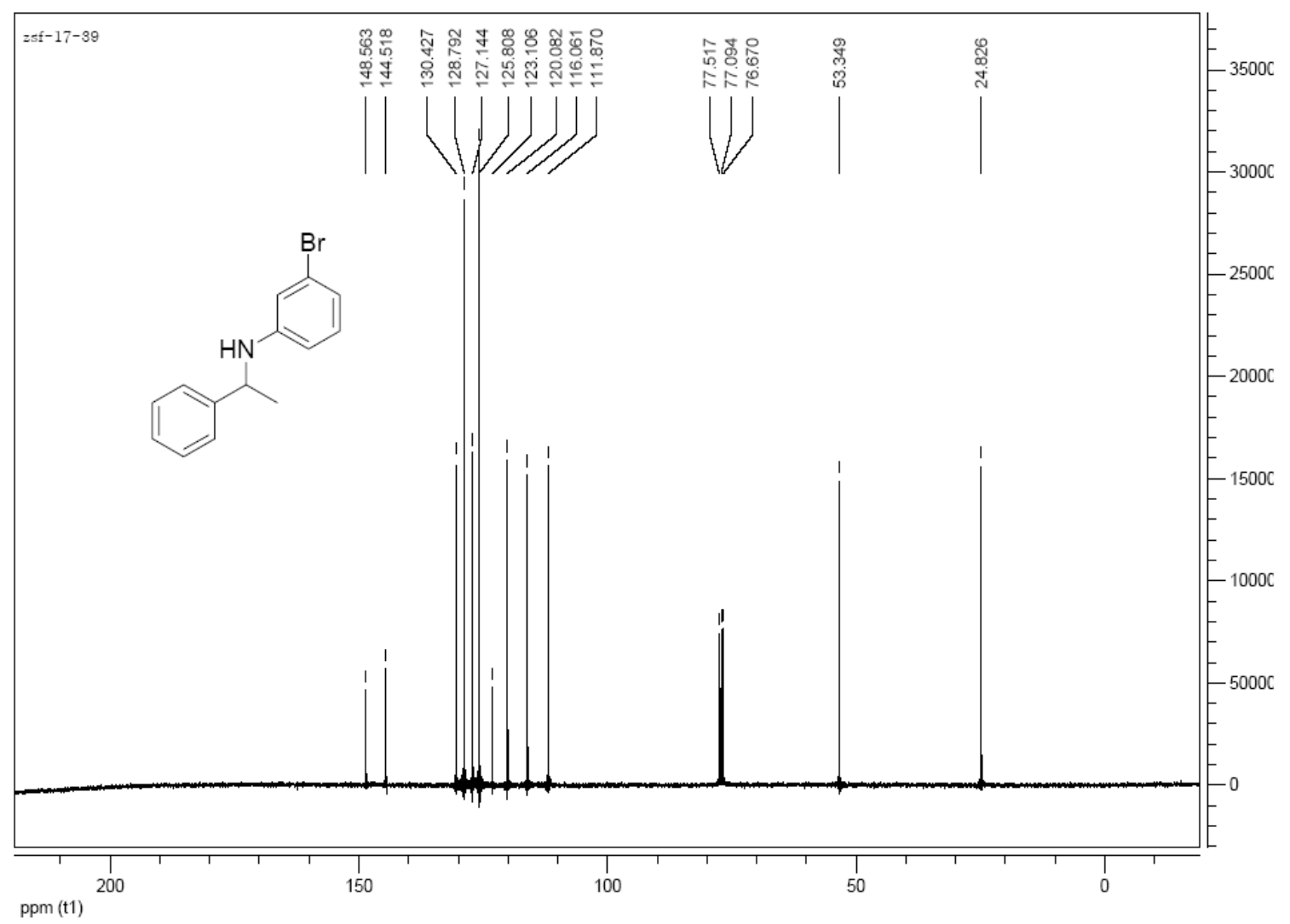



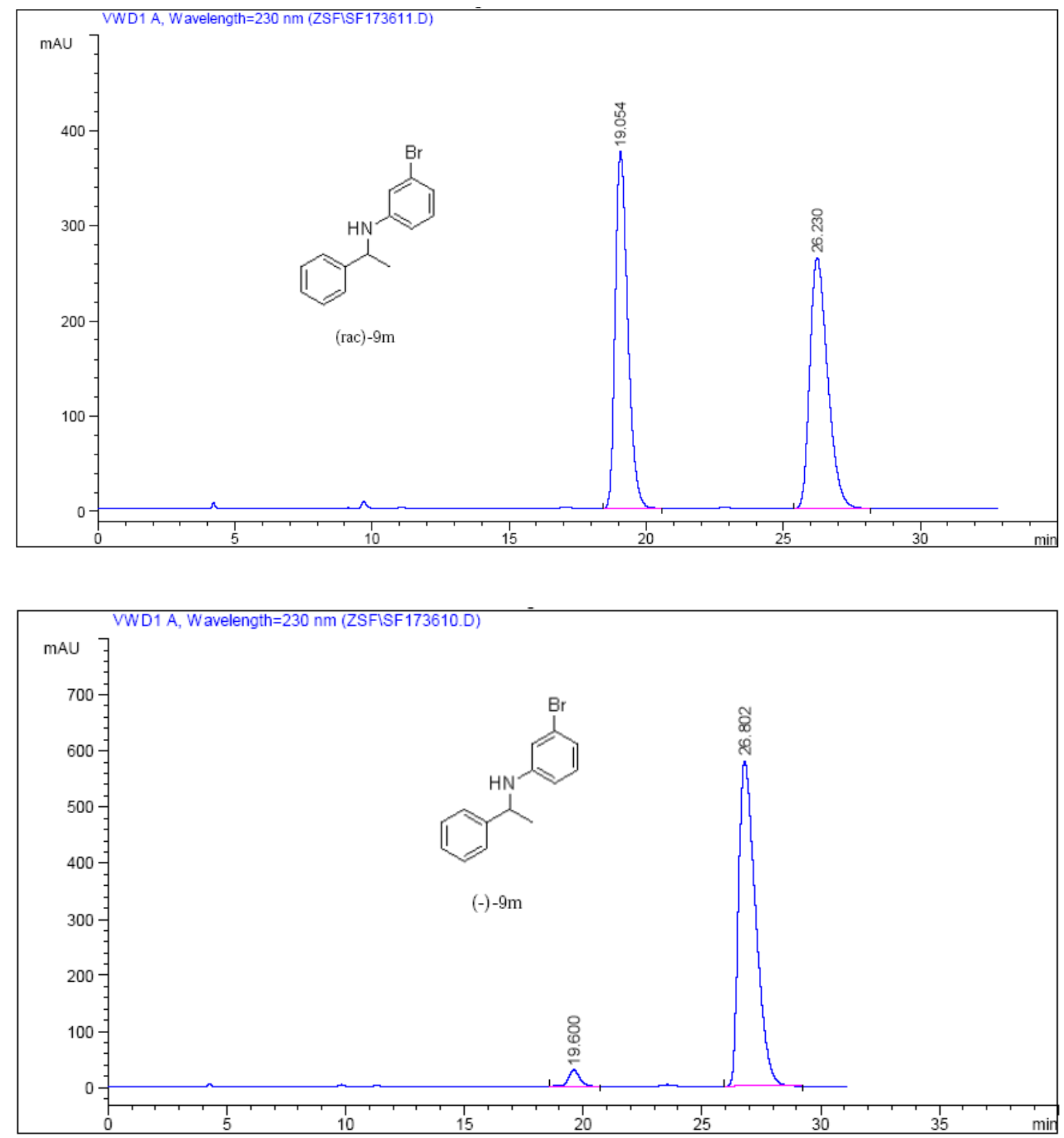

\section{X-Ray Diffraction Analyses of Structure of 9e}

The amine 9e with 91\% ee was dissolved in a mixed solvent of acetone and petroleum ether (boiling span $30-60{ }^{\circ} \mathrm{C}$ ) and evaporated under atmosphere. The colorless crystals (99.4\% ee) grow slowly which are suitable for the $\mathrm{X}$-ray diffraction analyses. The $\mathrm{X}$-ray diffraction analysis indicated that the absolute configuration of $\mathbf{9 e}$ is $R$. 


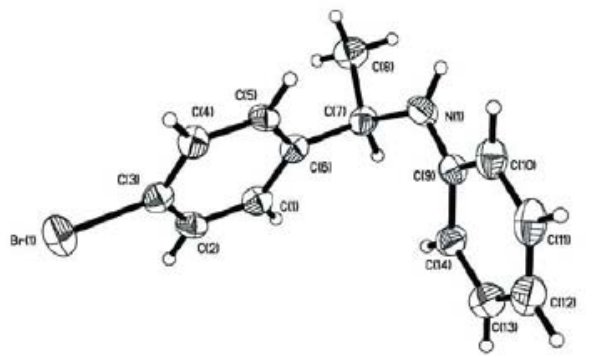

Table 4. Crystal data and structure refinement for $\mathbf{9 e}$

\begin{tabular}{|c|c|}
\hline Empirical formula & C14 H14 Br N \\
\hline Formula weight & 276.17 \\
\hline Temperature & 294(2) K \\
\hline Wavelength & $0.71073 \mathrm{~A}$ \\
\hline Crystal system, space group & Orthorhombic, $\quad$ P2(1)2(1)2(1) \\
\hline Unit cell dimensions & $\begin{array}{l}a=9.708(5) A \quad \text { alpha }=90 \text { deg. } \\
b=13.803(8) A \quad \text { beta }=90 \text { deg. } \\
c=18.973(10) A \quad \text { gamma }=90 \mathrm{deg} .\end{array}$ \\
\hline Volume & $2542(2) A^{\wedge} 3$ \\
\hline Z, Calculated density & 8, $\quad 1.443 \mathrm{Mg} / \mathrm{m}^{\wedge} 3$ \\
\hline Absorption coefficient & $3.206 \mathrm{~mm}^{\wedge}-1$ \\
\hline $\mathrm{F}(000)$ & 1120 \\
\hline Crystal size & $0.26 \times 0.22 \times 0.20 \mathrm{~mm}$ \\
\hline Theta range for data collection & 1.82 to 26.49 deg. \\
\hline Limiting indices & $-11<=\mathrm{h}<=12,-17<=\mathrm{k}<=17,-14<=\mathrm{l}<=23$ \\
\hline Reflections collected / unique & $14240 / 5192[\mathrm{R}(\mathrm{int})=0.0638]$ \\
\hline Completeness to theta $=26.49$ & $98.7 \%$ \\
\hline
\end{tabular}




\begin{tabular}{|l|l|}
\hline Absorption correction & Semi-empirical from equivalents \\
\hline Max. and min. transmission & 0.5665 and 0.4894 \\
\hline Refinement method & Full-matrix least-squares on F^2 \\
\hline Data / restraints / parameters & $5192 / 0 / 291$ \\
\hline Goodness-of-fit on F^2 & 0.982 \\
\hline Final R indices [I>2sigma(I)] & $\mathrm{R} 1=0.0396, \mathrm{wR} 2=0.0667$ \\
\hline R indices (all data) & $\mathrm{R} 1=0.1360, \mathrm{wR} 2=0.0894$ \\
\hline Absolute structure parameter & $0.007(12)$ \\
\hline Largest diff. peak and hole & 0.268 and -0.392 e.A^-3 \\
\hline
\end{tabular}

\section{References}

1 (a) Crabtree, R. H.; Quirk, J. M.; Felkin, H.; Fillebeen-Khan, T. Synth. React. Inorg. Met.-Org. Chem. 1982, 12, 407. (b) Marigo, M.; Marsich, N.; Farnetti, E. J. Mol. Chem. A: Chem. 2002, 187, 169.

2 (a) Brookhart, M.; Grant, B.; Volpe, A. F. Organometallics 1992, 11, 3920. (b) Nishida, H.; Takada, N.; Yoshimura, M.; Sonoda, T.; Kobayashi, H. Bull. Chem. Soc. Jpn. 1984, 57, 2600.

3 Samec, J. S. M.; Bäckvall, J.-E. Chem. Eur. J. 2002, 8, 2955.

4 Barluenga, J.; Fernandez, M. A.; Aznar, F.; Valdes, C. Chem. Eur. J. 2004, 10, 494.

5 Schnider, P.; Koch, G.; Pretot, R.; Wang, G.; Bohnen, M.; Kruger, C.; Pfaltz, A. Chem. Eur. J. 1997, 3, 887.

6 Vargas, S.; Rubio, M.; Suarez, A.; del Rio, D.; Alvarez, E.; Pizzano, A. Organometallics 2006, 25, 961.

7 Heutling, A.; Pohlk, F.; Doye, S. Chem. Eur. J. 2004, 10, 3059.

8 Li, K.; Horton, P. N.; Hursthouse, M. B.; Hii, K. K. J. Organometal. Chem. 2003, 665, 250.

9 Park, E. S.; Lee, J. H.; Kim, S. J.; Yoon, C. M. Synth. Commun. 2003, 33, 3387. 